\title{
TORIC GEOMETRY AND THE SEMPLE-NASH MODIFICATION
}

\author{
PEDRO D. GONZÁLEZ PÉREZ AND BERNARD TEISSIER
}

To Heisuke Hironaka on the occasion of his 80th birthday

\begin{abstract}
This paper proposes some material towards a theory of general toric varieties without the assumption of normality. Their combinatorial description involves a fan to which is attached a set of semigroups subjected to gluing-up conditions. In particular it contains a combinatorial construction of the blowing up of a sheaf of monomial ideals on a toric variety. In the second part this is used to show that iterating the Semple-Nash modification or its characteristic-free avatar provides a local uniformization of any monomial valuation of maximal rank dominating a point of a toric variety.
\end{abstract}

\section{INTRODUCTION}

In the first part of this paper we study abstract toric varieties without the assumption of normality. Since Sumihiro's Theorem on the existence of a covering of a toric variety by invariant affine varieties fails without the assumption of normality, we have to set the existence of such a covering as part of the definition of a toric variety. Then an abstract toric variety has a combinatorial description: it corresponds to certain semigroups in the convex duals of the cones of a fan, which satisfy a natural gluing-up condition. This generalizes the definition of 12 which concerns toric varieties equivariantly embedded in projective space. In spirit it is also a continuation of our previous work 13 on embedded normalization and embedded toric resolution of singularities of affine toric varieties. We can then define blowing-ups of sheaves of monomial ideals as toric varieties, and describe the corresponding operations on semigroups. We also provide the combinatorial description of torus-invariant Cartier divisors on a toric variety and the general versions of the classical criteria for ampleness and very-ampleness.

In the second part of the paper we use the description of blowing-ups given in the first part to show that one can obtain a local uniformization of a monomial valuation of maximal rank dominating a toric variety, by a finite number of iterations of the blowing-up of the logarithmic jacobian ideal introduced in 14. If the field is of characteristic zero, this blowing-up is isomorphic to the Semple-Nash modification. Recall that this is a canonical modification of a reduced equidimensional space which replaces each point by the set of limit positions of tangent spaces at nearby non-singular points. See the second part for details.

Our result is related in the special case of toric varieties to an interesting question apparently first asked by Semple in 27, that is, if the iteration of the Semple-Nash modification eventually resolves the singularities of an algebraic variety defined over a field of characteristic zero. One can present the relation of this conjectural resolution process with classical resolution of singularities as follows: two types of proper birational correspondences naturally associate a singular variety to a non-singular one: proper birational projections of an embedded non-singular algebraic variety to a smaller dimensional ambient space, and the taking of the envelope of a family of linear subspaces (of an affine or projective space) whose parameter space is a non singular algebraic variety. For example, a family of lines in the real plane parametrized by a circle, such as the Simson-Wallace lines of a triangle, parametrized by its circumscribed circle, have an envelope with singularities, generically with an odd number of cusps (see 35]). In the SimsonWallace lines case, it is a quartic with three cusps (see [30]). The natural map from the circle to the envelope is a homeomorphism and a local isomorphism outside of the cusps. Another

2000 Mathematics Subject Classification. 14M25, 14E15, $14 \mathrm{~B} 05$.

Key words and phrases. Toric geometry, Semple-Nash modification, logarithmic jacobian ideal.

González Pérez is supported by Severo Ochoa project SEV-2011-0087 and MTM2010-21740-C02-01 grants. Teissier is partially supported by grant MTM2007-64704. 
PEDRO D. GONZÁLEZ PÉREZ AND BERNARD TEISSIER

example is given by the discriminant hypersurface of a versal deformation of an isolated complex hypersurface singularity: it is the envelope of a family of complex hyperplanes parametrized by the critical locus, which is non-singular and is the normalization of the discriminant (see [33]) and therefore a resolution of singularities.

Hironaka's resolution shows that in characteristic zero all singularities may be created by the first process, and Semple-Nash resolution in general would show that at least all singularities of projective varieties in characteristic zero may be created by iterating a natural generalization of the second process, if we allow singular spaces as parameter spaces. Moreover it would produce a canonical process for local uniformization of valuations in characteristic zero, as our results here do for maximal rank monomial valuations on toric varieties in a characteristic-free manner.

Acknowledgements. We are grateful to Monique Lejeune-Jalabert for introducing us to the reference [27, to Ezra Miller for bringing to our attention the work of H.M. Thompson, to Michael Thaddeus for detecting errors in previous versions of this work.

We also mention that while this paper was in preparation, Mr. Daniel Duarte provided in 7. a proof of a result similar to our main result, a priori somewhat stronger and giving an effective bound on the number of steps required for desingularization, in the two-dimensional case. A preprint of Dima Grigoriev and Pierre Milman (see [17) also provided an approach to the Semple-Nash desingularization problem for toric varieties with explicit results in dimension 2. In particular it contains a version of our Lemma 12.1. We thank the referee for his careful work and helpful suggestions.

We also thank Patrice Philippon for his help, David Cox for his remarks and the Institut Mathématique de Jussieu and the Dpto. Álgebra, Universidad Complutense de Madrid for their hospitality.

\section{Part I: Toric varieties}

The purpose of this part is to develop the combinatorial theory of toric varieties without the assumption of normality. We refer to [6], [11, 21], 25], and 26] for background on normal toric varieties, and to the books of Oda-Miyake ([25]), Gel'fand, Kapranov, and Zelevinsky ([12]) and Sturmfels ([31) for certain classes of non necessarily normal toric varieties. We also point to previous work by H.M. Thompson towards the development of a general theory of toric varieties, see [36, 37] and, from the perspective of Log Schemes, 38. This part is also connected with the more general theory of monoid schemes recently developped by Cortiñas et al. in 4. We recommend [9] as a particularly accessible introduction to (normal) toric varieties, and also the recent book [5] of D. Cox, J. Little and H. Schenk on the subject.

\section{Semigroups And Semigroup Algebras}

The theory of affine toric varieties over a field $k$ is the geometric version of the theory of semigroup algebras over $k$. For part of the theory, one can omit the assumption that the semigroup is finitely generated, and replace the field $k$ by a commutative ring.

Definition 1.1. A (commutative) semigroup $\Gamma$ is a set equipped with an operation $+: \Gamma \times \Gamma \rightarrow \Gamma$ such that $\epsilon_{1}+\epsilon_{2}=\epsilon_{2}+\epsilon_{1}$, which satisfies the associativity property and is cancellative $\left(\epsilon_{1}+\epsilon_{2}=\epsilon_{1}+\epsilon_{3}\right.$ implies $\left.\epsilon_{2}=\epsilon_{3}\right)$. We shall assume that $\Gamma$ contains a zero element 0 such that $\epsilon+0=\epsilon$. We denote by $\mathbf{Z} \Gamma$ the group generated by $\Gamma$ (defined in a similar way as the field of fractions of an integral domain). To say that $\Gamma$ is cancellative means that the natural map of semigroups $\Gamma \rightarrow \mathbf{Z} \Gamma$ is injective. We say that $\Gamma$ is torsion free if the abelian group $\mathbf{Z} \Gamma$ is, which means that the only solution in $\mathbf{N}$ of an equation $m \gamma=m \gamma^{\prime}$ with $\gamma, \gamma^{\prime} \in \Gamma, \gamma \neq \gamma^{\prime}$, is $m=0$. Since $\Gamma$ is cancellative, it implies that the only solution in $\Gamma$ of an equation $m \gamma=n \gamma$ with $m, n \in \mathbf{N}, m \neq n$, is $\gamma=0$.

A system of generators of a semigroup is a subset $\left(\gamma_{i}\right)$ of $\Gamma$ such that each element of $\Gamma$ is a (finite) linear combination of the $\gamma_{i}$ with non negative integral coefficients. The elements of $\mathbf{Z} \Gamma$ are finite linear combinations of the $\gamma_{i}$ with integral coefficients. If the 
semigroup $\Gamma$ is cancellative, torsion free and finitely generated, the group $\mathbf{Z} \Gamma$ is a lattice so that $\Gamma$ is isomorphic to a finitely generated subsemigroup of a lattice $\mathbf{Z}^{d}$.

\section{Examples of semigroups:}

- Given finitely many coprime integers the set of all combinations of these integers with non negative integral coefficients is a subsemigroup $\Gamma$ of the semigroup $\mathbf{N}$ of integers, and $\mathbf{N} \backslash \Gamma$ is finite. In fact any semigroup of integers is finitely generated.

- Let $\left(s_{i}\right)_{i \geq 1}$ be a sequence of integers such that $s_{i} \geq 2$ for $i \geq 2$. Define a sequence of rational numbers $\gamma_{i}$ inductively by:

$$
\gamma_{1}=\frac{1}{s_{1}}, \quad \gamma_{i+1}=s_{i} \gamma_{i}+\frac{1}{s_{1} \ldots s_{i+1}} .
$$

The set of integral linear combinations of the $\gamma_{i}$ is a subsemigroup of $\mathbf{Q}_{\geq 0}$, which is not finitely generated. In fact the $\gamma_{i}$ form a minimal set of generators.

- Let $d$ be an integer and let $\check{\sigma}$ (the reason for the dual notation will appear below) be a convex cone of dimension $d$ in $\check{\mathbf{R}}^{d}$. Denote by $M$ the integral lattice of $\check{\mathbf{R}}^{d}$. Then the intersection $\check{\sigma} \cap M$ is a subsemigroup of the group $M$, which generates $M$ as a group. By a Theorem of Gordan, if the convex cone $\check{\sigma}$ is rational in the sense that it is the intersection of finitely many half spaces determined by hyperplanes with integral coefficients, then the semigroup $\check{\sigma} \cap M$ is finitely generated.

Definition 1.2. If $\Delta$ is a subsemigroup of $\Lambda$ the saturation of $\Delta$ in $\Lambda$ is the semigroup $\Theta$ consisting of those elements of $\Lambda$ which have a multiple in $\Delta$. The semigroup $\Delta$ is saturated in $\Lambda$ if $\Delta=\Theta$.

Lemma 1.3. Let $\check{\tau}$ be a rational convex cone in $\check{\mathbf{R}}^{d}$ for the lattice $M$. The semigroup $\check{\tau} \cap M$ is saturated in $M$ and the saturation of a subsemigroup $\Gamma$ of $M$ is $\check{\sigma} \cap M$ where $\check{\sigma}=\mathbf{R}_{\geq 0} \Gamma$ is the closed convex cone generated by $\Gamma$.

Proof. The first statement is clear. If $\mathbf{R}_{\geq 0} \Gamma=\check{\sigma}$, any element of $\check{\sigma} \cap M$ is a combination with rational coefficients of elements of $\Gamma$. Chasing denominators shows that an integral multiple of this element is in $\Gamma$. The converse is clear.

Definition 1.4. Let $\Gamma$ be a finitely generated commutative semigroup and $A$ a commutative ring. The semigroup algebra $A\left[t^{\Gamma}\right]$ of $\Gamma$ with coefficients in $A$ is the ring consisting of finite sums $\sum_{\gamma} a_{\gamma} t^{\gamma}$ with $a_{\gamma} \in A$, endowed with the multiplication law

$$
\left(\sum_{\gamma} a_{\gamma} t^{\gamma}\right)\left(\sum_{\delta} b_{\delta} t^{\delta}\right)=\sum_{\zeta}\left(\sum_{\gamma+\delta=\zeta} a_{\gamma} b_{\delta}\right) t^{\zeta}
$$

Proposition 1.5. If $\Gamma$ is a finitely generated subsemigroup of the lattice $M \subset \mathbf{R}^{d}$ such that $\mathbf{Z} \Gamma=M$ and $\check{\sigma}=\mathbf{R}_{>0} \Gamma$ is the rational convex cone generated by $\Gamma$, the integral closure of $k\left[t^{\Gamma}\right]$ in its field of fractions is $k\left[t^{\check{\sigma} \cap M}\right]$.

This follows directly from Lemma 1.3 .

Remark 1.6. Quite generally, if $k$ is a field the Krull dimension of $k\left[t^{\Gamma}\right]$ is equal to the rational rank of the semigroup $\Gamma$, which is the integer $\operatorname{dim}_{\mathbf{Q}} \Gamma \otimes_{\mathbf{Z}} \mathbf{Q}$ (see [34, Proposition $3.1)$.

Remark 1.7. If $\Gamma$ is a semigroup the ideal of $A\left[t^{\Gamma}\right]$ generated by the $\left(t^{\gamma}\right)_{\gamma \in \Gamma \backslash\{0\}}$ is non trivial if and only if the cone $\mathbf{R}_{\geq 0} \Gamma$ is strictly convex. If $k$ is a field, it is then a maximal ideal. We shall mostly be interested in the local study of the spectrum of semigroup algebras in the vicinity of the origin of coordinates, which corresponds precisely to that ideal. 
The semigroup algebra has the following universal property: any semigroup map from $\Gamma$ to the multiplicative semigroup of an $A$-algebra $B$ extends uniquely to an homomorphism $A\left[t^{\Gamma}\right] \rightarrow B$ of $A$-algebras.

An additive map of semigroups $\phi: \Gamma \rightarrow \Gamma^{\prime}$ induces a graded map of $A$-algebras $A[\phi]: A\left[t^{\Gamma}\right] \rightarrow A\left[t^{\Gamma^{\prime}}\right]$ which is injective (resp. surjective) if $\phi$ is. If the semigroup $\Gamma$ is torsion-free, the semigroup algebra $A\left[t^{\Gamma}\right]$ injects into $A\left[t^{\mathbf{Z}^{d}}\right]=A\left[t_{1}^{ \pm 1}, \ldots, t_{d}^{ \pm 1}\right]$ and therefore is an integral domain if $A$ is.

Proposition 1.8. Let $\Gamma, \Gamma^{\prime}$ be two semigroups. The map of A-algebras

$$
A\left[t^{\Gamma \times \Gamma^{\prime}}\right] \rightarrow A\left[u^{\Gamma}\right] \otimes_{A} A\left[v^{\Gamma^{\prime}}\right] ; \quad t^{\left(\gamma, \gamma^{\prime}\right)} \mapsto u^{\gamma} \otimes_{A} v^{\gamma^{\prime}}
$$

is an isomorphism.

Proof. This follows immediately from the universal property.

\section{Algebraic tori}

Let $k$ be a field. The multiplicative group $k^{*}$ of non-zero elements of $k$ is equipped with the structure of algebraic group over $k$, usually denoted by $\mathbf{G}_{m}:=\operatorname{Spec} k\left[t^{ \pm 1}\right]$. A $d$-dimensional algebraic torus over $k$ is an algebraic group isomorphic to a $\left(k^{*}\right)^{d}$.

If $M$ is a rank $d$ lattice then $T^{M}:=\operatorname{Spec} k\left[t^{M}\right]$ is an algebraic torus over $k$. If we fix a basis $m_{1}, \ldots, m_{d}$ of the lattice $M$ we get a group isomorphism

$$
\mathbf{Z}^{d} \rightarrow M, \quad a=\left(a_{1}, \ldots, a_{d}\right) \mapsto \sum_{i=1, \ldots d} a_{i} m_{i}
$$

and isomorphism of $k$-algebras $k\left[t_{1}^{ \pm 1}, \ldots, t_{d}^{ \pm 1}\right] \rightarrow k\left[t^{M}\right]$ which induces an isomorphism $T^{M}(k) \rightarrow\left(k^{*}\right)^{d}$.

Remark 2.1. More generally one can consider the scheme $\operatorname{Spec} A\left[t^{M}\right]$, which is an algebraic torus over $\operatorname{Spec} A$ for any commutative ring $A$.

A character of the torus $T(k)$ is a group homomorphism $T(k) \rightarrow k^{*}$. The set of characters Hom alg.groups $\left(T^{M}, k^{*}\right)$ of $T^{M}(k)$ is a multiplicative group isomorphic to the lattice $M$ by the homomorphism given by $m \mapsto t^{m}$ for $m \in M$. We identify the monomials $t^{m}$ of the semigroup algebra $k\left[t^{M}\right]$ with the characters of the torus.

By the universal property of the semigroup algebras applied to $k\left[t^{M}\right]$ we have a representation of $k$-rational points of $T^{M}$ as group homomorphisms:

$$
T^{M}(k)=\operatorname{Hom}_{\text {groups }}\left(M, k^{*}\right)=N \otimes_{\mathbf{z}} k^{*},
$$

where $N:=\operatorname{Hom}(M, \mathbf{Z})$ is the dual lattice of $M$. We denote by $\langle\rangle:, N \times M \rightarrow \mathbf{Z}$ the duality pairing between the lattices $N$ and $M$.

A one parameter subgroup of $T^{M}(k)$ is group homomorphism $k^{*} \rightarrow T^{M}(k)$. Any vector $\nu \in N$ gives rise to a one parameter subgroup $\lambda_{\nu}$ which maps $z \in k^{*}$ to the closed point of $T^{M}(k)$ given by the homomorphism of semigroups $M \rightarrow k^{*}, m \mapsto z^{\langle\nu, m\rangle}$. The set of one parameter subgroups Hom alg.groups $\left(k^{*}, T^{M}\right)$ forms a multiplicative group, which is isomorphic to $N$ by the homomorphism given by $\nu \mapsto \lambda_{\nu}$.

\section{Affine toric VARIETiES}

In this section we consider a finitely generated subsemigroup $\Gamma$ of a free abelian group $M$ of rank $d$. We assume in addition that the group $\mathrm{Z} \Gamma$ generated by $\Gamma$ is equal to $M$. We denote by $N$ the dual lattice of $M$. We introduce some useful notations. 
Notation 3.1. We denote by $M_{\mathbf{R}}$ the $d$-dimensional real vector space $M \otimes_{\mathbf{Z}} \mathbf{R}$. The semigroup $\Gamma$, viewed in $M_{\mathbf{R}}$, spans the cone $\mathbf{R}_{\geq 0} \Gamma \subset M_{\mathbf{R}}$ which we denote also by $\check{\sigma}$. The dual cone of $\check{\sigma}$ is the cone $\sigma:=\left\{\nu \in N_{\mathbf{R}} \mid\langle\nu, \gamma\rangle \geq 0, \forall \gamma \in \check{\sigma}\right\}$. We use the notation $\tau \leq \sigma$ to indicate that $\tau$ is a face of $\sigma$. Any face of $\check{\sigma}$ is of the form $\check{\sigma} \cap \tau^{\perp}$ for a unique face $\tau$ of $\sigma$, where $\tau^{\perp}$ is the linear subspace $\left\{\gamma \in M_{\mathbf{R}} \mid\langle\nu, \gamma\rangle=0, \forall \nu \in \tau\right\}$.

Let $\gamma_{1}, \ldots, \gamma_{r}$ be generators of $\Gamma$. Then the semigroup $\Gamma$ is the image of $\mathbf{N}^{r} \subset \mathbf{Z}^{r}$ by the surjective linear map $b: \mathbf{Z}^{r} \rightarrow M$ determined by $b\left(e_{i}\right)=\gamma_{i}$ where the $e_{i}, 1 \leq i \leq r$ form the canonical basis of $\mathbf{N}^{r}$. The kernel $\mathcal{L}$ of $b$ is isomorphic to $\mathbf{Z}^{r-d}$.

Let us consider the map of semigroup algebras associated to the map $b \mid \mathbf{N}^{r}: \mathbf{N}^{r} \rightarrow \mathbf{Z}^{d}$, whose image is $\Gamma$. It is a map of $A$-algebras $A\left[U_{1}, \ldots, U_{r}\right] \rightarrow A\left[t_{1}^{ \pm 1}, \ldots, t_{d}^{ \pm 1}\right]$. Its image is the subalgebra $A\left[t^{\Gamma}\right]$ of $A\left[t_{1}^{ \pm 1}, \ldots, t_{d}^{ \pm 1}\right]$.

An element $m \in \mathbf{Z}^{r}$ can be written uniquely $m=m_{+}-m_{-}$where $m_{+}$and $m_{-}$have non negative entries and disjoint support.

By construction, the kernel of the surjection $A\left[U_{1}, \ldots, U_{r}\right] \rightarrow A\left[t^{\Gamma}\right]$ is the ideal generated by the binomials $\left(U^{m_{+}}-U^{m_{-}}\right)$where $b\left(m_{+}\right)=b\left(m_{-}\right)$. It is the toric ideal associated to the map $b$. Note that it is not in general generated by the binomials associated to a basis of $\mathcal{L}$. Since the algebra $A\left[t^{\Gamma}\right]$ is an integral domain if $A$ is, the toric ideal is a prime ideal in that case.

Conversely, assuming now that $A$ is an algebraically closed field $k$, an ideal generated by binomials in $k\left[U_{1}, \ldots, U_{r}\right]$ is called a binomial ideal. Those ideals are studied in [8], where it is shown that a prime binomial ideal $I \subset k\left[U_{1}, \ldots, U_{r}\right]$ gives rise to a semigroup algebra $k\left[U_{1}, \ldots, U_{r}\right] / I \simeq k\left[t^{\Gamma}\right]$, where $\Gamma=\mathbf{N}^{r} / \sim$, and $\sim$ is an equivalence relation associated to the binomial relations. The affine toric variety $T^{\Gamma}:=\operatorname{Spec} k\left[t^{\Gamma}\right]$ is the subvariety of the affine space $\mathbf{A}^{r}(k)$ defined by the binomial equations generating the toric ideal. By the universal property of the semigroup algebra, there is a bijection

\{Closed points of Spec $\left.k\left[t^{\Gamma}\right]\right\} \leftrightarrow$ \{semigroup homomorphisms $\left.\Gamma \rightarrow k\right\}$,

where $k$ is considered as a semigroup with respect to multiplication (in particular $0 \in \Gamma$ goes to $1 \in k$ ).

In particular, the torus $T^{M}(k)=\operatorname{Hom}_{\text {groups }}\left(M, k^{*}\right)$ is embedded in $T^{\Gamma}$, as the principal open set where $t^{\gamma_{1}} \cdots t^{\gamma_{r}} \neq 0$.

From the description of closed points of $T^{\Gamma}$ in terms of homomorphisms of semigroups we have an action of the torus $T^{M}(k)$ on $T^{\Gamma}(k)$. Another way to describe this action, which shows that it is algebraic, is to say that thanks to the universal property of semigroup algebras it corresponds to the composed map of $k$-algebras

$$
k\left[t^{\Gamma}\right] \rightarrow k\left[t^{\Gamma}\right] \otimes_{k} k\left[t^{\Gamma}\right] \rightarrow k\left[t^{M}\right] \otimes_{k} k\left[t^{\Gamma}\right]
$$

where the first map is determined by $t^{\gamma} \mapsto t^{\gamma} \otimes_{k} t^{\gamma}$ and the second by the inclusion $\Gamma \subset M$. The corresponding map $T^{M} \times T^{\Gamma} \rightarrow T^{\Gamma}$ is the action.

Let us now seek the invariant subsets of $T^{\Gamma}$ under the torus action.

Definition 3.2. Given a semigroup $\Gamma$, a subsemigroup $F \subset \Gamma$ is a face of $\Gamma$ if whenever $x, y \in \Gamma$ satisfy $x+y \in F$, then $x$ and $y$ are in $F$.

Let us remark that this condition is equivalent to the fact that the vector space of finite sums $\sum_{\delta \in \Gamma \backslash F} a_{\delta} t^{\delta}$ is in fact a prime ideal $I_{F}$ of $k\left[t^{\Gamma}\right]$. It also implies that $\Gamma \backslash F$ is a subsemigroup of $\Gamma$ (which in general is not finitely generated) and that the Minkowski sum $\Gamma+(\Gamma \backslash F)$ is contained in $\Gamma \backslash F$.

Lemma 3.3. The faces of the semigroup $\Gamma$ are of the form $\Gamma \cap \tau^{\perp}$, for $\tau \leq \sigma$.

Proof. Let $F$ be a face of the semigroup $\Gamma$. Then there is a face $\check{\sigma} \cap \tau^{\perp}$ of $\check{\sigma}$ which contains $F$ and is of minimal dimension. Then $F$ is also a face of the semigroup $\Gamma \cap \tau^{\perp}$ 
and there is an element $\gamma_{0} \in F$ which belongs to the relative interior of the cone $\check{\sigma} \cap \tau^{\perp}$. Under these conditions is enough to prove that if $\tau=0$ then $F=\Gamma$.

Notice that if $\gamma \in \Gamma$ and if $(\gamma+\Gamma) \cap \mathbf{Z}_{\geq 0} \gamma_{0} \neq \emptyset$ then $\gamma \in F$ since $F$ is a face and $\gamma_{0} \in F$. By Theorem 1.9 [20] there is $\delta_{0} \in \Gamma \cap \operatorname{int}(\check{\sigma})$ such that $\delta_{0}+\check{\sigma} \cap M \subset \Gamma$. We deduce that the intersection $\left(\gamma+\delta_{0}+\check{\sigma} \cap M\right) \cap \mathbf{Z}_{\geq 0} \gamma_{0}$ is non-empty, for any $\gamma \in \Gamma$, since $\gamma_{0} \in \operatorname{int}(\check{\sigma}) \cap \Gamma$.

Notation 3.4. If $\tau \leq \sigma$ the set $\Gamma \cap \tau^{\perp}$ is a subsemigroup of finite type of $\Gamma$. If $\tau \leq \sigma$ the lattice $M(\tau, \Gamma)$ spanned by $\Gamma \cap \tau^{\perp}$ is a sublattice of finite index of $M(\tau):=M \cap \tau^{\perp}$.

Remark 3.5. The torus of the affine toric variety $T^{\Gamma \cap \tau^{\perp}}$ is $T^{M(\tau, \Gamma)}$. If $A$ is a commutative ring, the homomorphism of $A$-algebras $A[\Gamma] \rightarrow A\left[\Gamma \cap \tau^{\perp}\right] \cong A[\Gamma] / I_{\Gamma \cap \tau^{\perp}}$, is surjective and defines a closed embedding

$$
i_{\tau}: T^{\Gamma \cap \tau^{\perp}} \hookrightarrow T^{\Gamma}
$$

over $\operatorname{Spec} A$. If $k=A$ the image by the embedding $i_{\tau}$ of a closed point $u \in T^{\Gamma \cap \tau^{\perp}}(k)$ (or $u \in T^{M(\tau, \Gamma)}(k)$ ) is the semigroup homomorphism $i_{\tau}(u): \Gamma \rightarrow k$ given by

$$
\gamma \mapsto\left\{\begin{array}{cc}
u(\gamma) & \text { if } \gamma \in \tau^{\perp} \\
0 & \text { otherwise. }
\end{array}\right.
$$

Proposition 3.6. The map

$$
\tau \mapsto \operatorname{orb}(\tau, \Gamma):=i_{\tau}\left(T^{M(\tau, \Gamma)}\right) \quad\left(\operatorname{resp} . \tau \mapsto i_{\tau}\left(T^{\Gamma \cap \tau^{\perp}}\right)\right)
$$

defines a bijection (resp. inclusion-reversing bijection) between the faces of $\sigma$ and the orbits (resp. the closures of the orbits) of the torus action on $T^{\Gamma}$.

Proof. Let $u: \Gamma \rightarrow k$ be a semigroup homomorphism. Then $u^{-1}\left(k^{*}\right)$ is a face of $\Gamma$, hence of the form $\Gamma \cap \tau^{\perp}$ for some face $\tau$ of $\sigma$. Any such $u$ extends in a unique manner to a group homomorphism $M(\tau, \Gamma) \rightarrow k^{*}$ defining an element of the torus $T^{M(\tau, \Gamma)}$ of the affine toric variety $T^{\Gamma \cap \tau^{\perp}}$. Conversely, given a group homomorphism $u: M(\tau, \Gamma) \rightarrow k^{*}$ we define a semigroup homomorphism $i_{\tau}(u): \Gamma \rightarrow k$ as indicated above.

It follows that the orbit of the point defined by $u$ by the action of $T^{M}$ coincides with the image by $i_{\tau}$ of the orbit $T^{M(\tau, \Gamma)}$ of the point $u_{\mid \Gamma \cap \tau^{\perp}}: \Gamma \cap \tau^{\perp} \rightarrow k^{*}$ on the toric variety $T^{\Gamma \cap \tau^{\perp}}$. The rest of the assertion follows from Remark 3.5.

The partition induced by the orbits of the torus action on $T^{\Gamma}$ is of the form:

$$
T^{\Gamma}=\bigsqcup_{\tau \leq \sigma} \operatorname{orb}(\tau, \Gamma)
$$

Proposition 3.7. If $X$ is an affine toric variety with torus $T^{M}$ then $X$ is $T^{M}$-equivariantly isomorphic to $T^{\Gamma}$, where $\Gamma \subset M$ a semigroup of finite type such that $\mathbf{Z} \Gamma=M$.

Proof. This is well-known (see Proposition 2.4, Chapter 5 of [12]).

We characterize the affine $T^{M}$-invariant open subsets of $T^{\Gamma}$.

Definition 3.8. For any face $\tau$ of $\sigma$ the set

$$
\Gamma_{\tau}:=\Gamma+M(\tau, \Gamma)
$$

is a semigroup of finite type generating the lattice $M$.

Notice that the cone $\mathbf{R}_{\geq 0} \Gamma_{\tau}$ is equal to $\check{\tau}$ and if $\tau \leq \sigma$ the set $\operatorname{int}\left(\check{\sigma} \cap \tau^{\perp}\right) \cap \Gamma$ is non empty (int denotes relative interior).

\section{Lemma 3.9.}

i. The minimal face of the semigroup $\Gamma$ is a sublattice of $M$ equal to $\Gamma \cap \sigma^{\perp}$. 
ii. For any $m \in \Gamma$ in the relative interior of $\left(\check{\sigma} \cap \tau^{\perp}\right)$ we have that

$$
\Gamma_{\tau}=\Gamma+\mathbf{Z}_{\geq 0}(-m)
$$

iii. If $\tau \leq \theta \leq \sigma$ we have that $M\left(\tau, \Gamma_{\theta}\right)=M\left(\tau, \Gamma_{\tau}\right)$ and $\Gamma_{\tau}=\Gamma_{\theta}+M\left(\tau, \Gamma_{\theta}\right)$.

Proof. i. By Lemma 3.3 the correspondence $\tau \mapsto \Gamma \cap \tau^{\perp}$ is a bijection between the faces of the cone $\sigma$ and the faces of the semigroup $\Gamma$. By duality the minimal face of $\Gamma$ is equal to $\Gamma \cap \sigma^{\perp}$. It is enough to prove that if $\Gamma$ is a semigroup such that $\mathbf{Z} \Gamma=M$ and $\mathbf{R}_{\geq 0} \Gamma=M_{\mathbf{R}}$ then $\Gamma=M$. Since $M$ is the saturation of $\Gamma$ the assertion reduces to the case of rank one semigroups, for which it is elementary by Bezout identity.

ii. If $m \in \operatorname{int}\left(\check{\sigma} \cap \tau^{\perp}\right) \cap \Gamma$ then the semigroup $\Gamma+\mathbf{Z}_{\geq 0}(-m) \subset M$ spans the cone $\check{\tau}=$ $\check{\sigma}+\tau^{\perp} \subset M_{\mathbf{R}}$. By i. the minimal face of this semigroup is the lattice $\left(\Gamma+\mathbf{Z}_{\geq 0}(-m)\right) \cap \tau^{\perp}$ which coincides by definition with the lattice $M(\tau, \Gamma)$.

iii. The lattices $M\left(\tau, \Gamma_{\theta}\right)$ and $M(\tau, \Gamma)$ are both generated by $\Gamma \cap \tau^{\perp}$ hence are equal. We have that $\Gamma_{\tau}=\Gamma_{\theta}+M\left(\tau, \Gamma_{\theta}\right)$ since $\theta^{\perp} \subset \tau^{\perp}$.

Lemma 3.10. If $\tau \leq \sigma$ the inclusion of semigroups $\Gamma \subset \Gamma_{\tau}$ determines a $T^{M}$-equivariant embedding $T^{\Gamma_{\tau}} \subset T^{\Gamma}$ as an affine open set. Conversely, if $X \subset T^{\Gamma}$ is a $T^{M}$-equivariant embedding of an affine open set then there is a unique $\tau \leq \sigma$ such that $X$ is $T^{M}$ equivariantly isomorphic to $T^{\Gamma_{\tau}}$.

Proof. By Lemma 3.9 we have that $\Gamma_{\tau}=\Gamma+\mathbf{Z}_{>0}(-m)$. More generally if $\gamma \in \Gamma$ and $f=t^{\gamma}$, the localization $T_{f}^{\Gamma}=\operatorname{Spec} k[\Gamma]_{f}$ is equal to $T^{\Gamma+(-\gamma) \mathbf{Z}_{\geq 0}}$ and it is embedded in $T^{\Gamma}$ as a principal open set.

Conversely, an affine $T^{M}$-invariant open subset of $T^{\Gamma}$ is an affine toric variety for the torus $T^{M}$ hence it is of the form $T^{\Lambda}$, for $\Lambda \subset M$ a subsemigroup of finite type, such that $\mathbf{Z} \Lambda=M$ (see Proposition 3.7). We denote the cone $\mathbf{R}_{>0} \Lambda$ by $\check{\theta}$. Since the embedding $T^{\Lambda} \subset T^{\Gamma}$ is $T^{M}$ equivariant it is defined by the inclusion of algebras $k\left[t^{\Gamma}\right] \rightarrow k\left[t^{\Lambda}\right]$ corresponding to the inclusion of semigroups $\Gamma \subset \Lambda$. We deduce that $\check{\sigma} \subset \check{\theta}$ and hence that $\theta \subset \sigma$ by duality. We prove that if $\tau$ is the smallest face of $\sigma$ which contains $\theta$ then $\Lambda=\Gamma_{\tau}$. It is enough to prove that if $\operatorname{int}(\theta) \cap \operatorname{int}(\sigma) \neq \emptyset$ then $\Lambda=\Gamma$.

Notice that the lattice $F=\sigma^{\perp} \cap M$ is the minimal face of $\Gamma$ and the prime ideal $I_{F}$ of $k\left[t^{\Gamma}\right]$ defines the orbit $\operatorname{orb}(\sigma, \Gamma)$, which is embedded as a closed subset of $T^{\Gamma}$. Let us consider a vector $\nu$ such that $\nu \in \operatorname{int}(\theta) \cap \operatorname{int}(\sigma)$. Then we get that $\sigma^{\perp} \cap M=\check{\sigma} \cap \nu^{\perp} \cap M$ is contained in $\check{\theta} \cap \nu^{\perp} \cap M=\theta^{\perp} \cap M$ hence $\Gamma \backslash\left(\sigma^{\perp} \cap M\right)$ is contained in $\Lambda \backslash\left(\theta^{\perp} \cap M\right)$ and therefore $1 \notin I_{F} k\left[t^{\Lambda}\right]$. Since $T^{\Lambda} \subset T^{\Gamma}$ is an open immersion $\operatorname{orb}(\sigma, \Gamma)$ is contained in $T^{\Lambda}$. By (1) and Proposition 3.6 the closure of any orbit contained $T^{\Gamma}$ contains $\operatorname{orb}(\sigma, \Gamma)$ thus $T^{\Gamma} \subset T^{\Lambda}$.

Remark 3.11. The immersion of $T^{M}$-invariant affine open subsets is compatible with normalization. By Lemma 3.10 any $T^{M}$-invariant affine open set of $T^{\Gamma}$ is of the form $T_{f}^{\Gamma}$ for $f=t^{\gamma}, \gamma \in \Gamma$. Then the following diagram commutes:

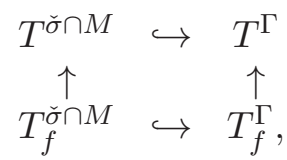

since $\Gamma+(-\gamma) \mathbf{Z}_{\geq 0}$ is saturated in $\check{\sigma} \cap M+(-\gamma) \mathbf{Z}_{\geq 0}$. The vertical arrows are embeddings as principal open sets while the horizontal arrows are normalization maps (see Proposition 1.5).

\section{TORIC VARIETIES}

Given a finite dimensional lattice $N$, recall that a fan is a finite set $\Sigma$ of strictly convex polyhedral cones of the real vector space $N_{\mathbf{R}}$ which are rational for the lattice 
$N$, such that if $\sigma \in \Sigma$ any face $\tau$ of $\sigma$ belongs to $\Sigma$ and if $\sigma, \sigma^{\prime} \in \Sigma$ the cone $\tau=\sigma \cap \sigma^{\prime}$ is in $\Sigma$. If $j \geq 0$ is an integer the subset of $\Sigma(j)$ of $j$-dimensional cones of $\Sigma$ is called the $j$-skeleton of the fan. The support of the fan $\Sigma$ is the set $|\Sigma|=\cup_{\sigma \in \Sigma} \sigma \subset N_{\mathbf{R}}$.

We give first a combinatorial definition of toric varieties.

Definition 4.1. A toric variety is given by the datum of a triple $(N, \Sigma, \Gamma)$ consisting of lattice $N$, a fan $\Sigma$ in $N_{\mathbf{R}}$ and a family of finitely generated subsemigroups $\Gamma=\left\{\Gamma_{\sigma} \subset\right.$ $\check{\sigma} \cap M\}_{\sigma \in \Sigma}$ of a lattice $M=\operatorname{Hom}(N, \mathbf{Z})$ such that:

i. $\mathbf{Z} \Gamma_{\sigma}=M$ and $\mathbf{R}_{\geq 0} \Gamma_{\sigma}=\check{\sigma}$, for $\sigma \in \Sigma$.

ii. $\Gamma_{\tau}=\Gamma_{\sigma}+M\left(\tau, \Gamma_{\sigma}\right)$, for a each $\sigma \in \Sigma$ and any face $\tau$ of $\sigma$.

The corresponding toric variety $T_{\Sigma}^{\Gamma}$ is the union of the affine varieties $T^{\Gamma_{\sigma}}$ for $\sigma \in \Sigma$ where for any pair $\sigma, \sigma^{\prime}$ in $\Sigma$ we glue up $T^{\Gamma_{\sigma}}$ and $T^{\Gamma_{\sigma^{\prime}}}$ along their common open affine variety $T^{\Gamma_{\sigma \cap \sigma^{\prime}}}$.

Remark 4.2. The lattice $N$ in the triple $(N, \Sigma, \Gamma)$ is determined by $\Gamma$. We recall it by convenience. We omit the reference to the lattice $N$ in the notation $T_{\Sigma}^{\Gamma}$.

Remark 4.3. This definition is consistent with the case of affine toric varieties. Let $T^{\Gamma}$ be an affine toric variety in the sense of Section 3. If $\sigma^{\prime}:=\{\tau \mid \tau \leq \sigma\}$ and $\Gamma^{\prime}:=\left\{\Gamma_{\tau} \mid \tau \leq \sigma\right\}$, where $\Gamma_{\tau}$ is the semigroup defined by (2) for $\tau \leq \sigma$ then the conditions i. and ii. are satisfied by Lemma 3.9. Then $T^{\Gamma}$ is $T^{M}$-equivariantly isomorphic to $T_{\sigma^{\prime}}^{\Gamma^{\prime}}$.

Remark 4.4. A triple $(N, \Sigma, \Gamma)$ determines similarly a toric scheme over $\operatorname{Spec} A$, for any commutative ring $A$.

Lemma 4.5. Let $(\Sigma, \Gamma)$ as in Definition 4.1 define a toric variety $T_{\Sigma}^{\Gamma}$. Then we have:

i. If $\sigma, \theta \in \Sigma$ and if $\tau=\sigma \cap \theta$ then $\Gamma_{\tau}=\Gamma_{\sigma}+\Gamma_{\theta}$.

ii. The variety $T_{\Sigma}^{\Gamma}$ is separated.

Proof. The intersection $\tau=\sigma \cap \theta$ is a face of both $\sigma$ and $\theta$. By Lemma 3.9 we have that $M\left(\tau, \Gamma_{\tau}\right)=M\left(\tau, \Gamma_{\sigma}\right)=M\left(\tau, \Gamma_{\theta}\right)$. By axiom i. in the Definition 4.1 we get $\Gamma_{\theta}, \Gamma_{\sigma} \subset \Gamma_{\tau}$ and $\Gamma_{\theta}+\Gamma_{\sigma} \subset \Gamma_{\tau}$. Conversely, by the separation lemma for polyhedral cones, for any $u \in \operatorname{int}(\check{\sigma} \cap(-\check{\theta}))$ we have that $\tau=\sigma \cap u^{\perp}=\theta \cap u^{\perp}$. Notice that we can assume that $u \in \Gamma_{\sigma} \cap\left(-\Gamma_{\theta}\right) \cap \operatorname{int}(\check{\sigma} \cap(-\check{\theta})) \neq \emptyset$. Then by Lemma 3.9 we obtain $\Gamma_{\tau}=\Gamma_{\sigma}+\mathbf{Z}_{\geq 0}(-u)$. Hence $\Gamma_{\tau}$ is contained in $\Gamma_{\sigma}+\Gamma_{\theta}$ since $-u \in \Gamma_{\theta}$.

The homomorphism $k\left[t^{\Gamma_{\theta}}\right] \otimes_{k} k\left[t^{\Gamma_{\sigma}}\right] \rightarrow k\left[t^{\Gamma_{\tau}}\right]$ which sends $t^{\gamma} \otimes t^{\gamma^{\prime}} \mapsto t^{\gamma+\gamma^{\prime}}$ is surjective since $\Gamma_{\sigma}+\Gamma_{\theta}=\Gamma_{\tau}$. In geometric terms this implies that the diagonal map $T^{\Gamma_{\tau}} \rightarrow$ $T^{\Gamma_{\theta}} \times T^{\Gamma_{\sigma}}$ is a closed embedding for any $\theta, \sigma \in \Sigma$ with $\tau=\theta \cap \tau$, hence the variety $T_{\Sigma}^{\Gamma}$ is separated (see Chapter 2 of [18]).

Remark 4.6. The morphisms corresponding to the inclusions $k\left[t^{\Gamma_{\sigma}}\right] \rightarrow k\left[t^{\Gamma_{\sigma}+\Gamma_{\sigma^{\prime}}}\right]$ are open embeddings compatible with the normalization maps. The normalization of the toric variety $T_{\Sigma}^{\Gamma}$ is the toric variety $T_{\Sigma}$ corresponding to the fan $\Sigma$ and the normalization map is obtained by gluing-up normalizations $T_{\sigma}:=T^{\check{\sigma} \cap M}$ of the charts $T^{\Gamma_{\sigma}}$, for $\Gamma_{\sigma} \in \Gamma$ and $\sigma \in \Sigma$.

Lemma 4.7. Let $\lambda_{v}$ be a one-parameter subgroup of the torus $T^{M}$ for some $v \in N$. Then $\lim _{z \rightarrow 0} \lambda_{v}(z)$ exists in the toric variety $T_{\Sigma}^{\Gamma}$ if and only if $v$ belongs to $|\Sigma| \cap N$.

Proof. The statement is well-known in the normal case (see Proposition 1.6 [26]). The normalization map $n: T_{\Sigma} \rightarrow T_{\Sigma}^{\Gamma}$ is an isomorphism over the torus $T^{M}$. If $\lambda_{v}: k^{*} \rightarrow$ $T^{M} \subset T_{\Sigma}^{\Gamma}$ is a one-parameter subgroup defined by $v \in N$ it lifts to the normalization, i.e., there is a morphism $\bar{\lambda}_{v}: k^{*} \rightarrow T^{M} \subset T_{\Sigma}$ in such a way that $n \circ \bar{\lambda}_{v}=\lambda_{v}$. Since the normalization is a proper morphism we get by the valuative criterion of properness that $\lim _{z \rightarrow 0} \lambda_{v}(z)$ exists in the toric variety $T_{\Sigma}^{\Gamma}$ if and only if $\lim _{z \rightarrow 0} \bar{\lambda}_{v}(z)$ exists in $T_{\Sigma}$. 
Lemma 4.8. Let $T_{\Sigma}^{\Gamma}$ be a toric variety. Then the map

$$
\tau \mapsto \operatorname{orb}\left(\tau, \Gamma_{\tau}\right):=i_{\tau}\left(T^{M\left(\tau, \Gamma_{\tau}\right)}\right)
$$

defines a bijection between the faces of $\Sigma$ and the orbits of the torus action on $T_{\Sigma}^{\Gamma}$.

Proof. This is consequence of the definitions and Lemma 3.6.

In order to illustrate the combinatorial definition of a toric variety we describe the orbit closures as toric varieties.

Notation 4.9. If $\tau \in \Sigma$ we denote by $N_{\tau}$ the sublattice of $N$ spanned by $\tau \cap N$ and by $N(\tau)$ the quotient $N / N_{\tau}$. The lattice $N(\tau)$ is the dual lattice of $M(\tau)=M \cap \tau^{\perp}$. Since $M\left(\tau, \Gamma_{\tau}\right)$ is a sublattice of finite index $i\left(\tau, \Gamma_{\tau}\right)$ of $M(\tau)$ then the dual lattice $N\left(\tau, \Gamma_{\tau}\right)$ of $M\left(\tau, \Gamma_{\tau}\right)$ contains $N(\tau)$ as a sublattice of finite index equal to $i\left(\tau, \Gamma_{\tau}\right)$.

If $\sigma \in \Sigma$ and $\tau \leq \sigma$ the image $\sigma(\tau)$ of $\sigma$ in $N(\tau)_{\mathbf{R}}=N_{\mathbf{R}} /\left(N_{\tau}\right)_{\mathbf{R}}$ is a polyhedral cone, rational for the lattice $N\left(\tau, \Gamma_{\tau}\right)$. The set $\Sigma(\tau):=\{\sigma(\tau) \mid \sigma \in \Sigma, \tau \leq \sigma\}$ is a fan in $N(\tau)_{\mathbf{R}}$. If $\sigma(\tau) \in \Sigma(\tau)$ we set $\Gamma_{\sigma(\tau)}:=\Gamma_{\sigma} \cap \tau^{\perp}$. The set $\sigma(\tau) \subset N(\tau)_{\mathbf{R}}$ is the dual cone of the cone spanned by $\Gamma_{\sigma} \cap \tau^{\perp}$ in $M(\tau)_{\mathbf{R}}$. Let us denote by $\Gamma(\tau)$ the set $\left\{\Gamma_{\sigma(\tau)} \mid \sigma(\tau) \in \Sigma(\tau)\right\}$

Lemma 4.10. Let $T_{\Sigma}^{\Gamma}$ be a toric variety. If $\tau \in \Sigma$ the triple $\left(N\left(\tau, \Gamma_{\tau}\right), \Sigma(\tau), \Gamma(\tau)\right)$ defines a toric variety $T_{\Sigma(\tau)}^{\Gamma(\tau)}$. We have a closed embedding $i_{\tau}: T_{\Sigma(\tau)}^{\Gamma(\tau)} \rightarrow T_{\Sigma}^{\Gamma}$. The map

$$
\tau \mapsto i_{\tau}\left(T_{\Sigma(\tau)}^{\Gamma(\tau)}\right)
$$

defines a bijection between the faces of $\Sigma$ and orbit closures of the action of $T^{M}$ on $T_{\Sigma}^{\Gamma}$.

Proof. If $\tau$ is not a face of $\sigma$, for $\sigma \in \Sigma$ then $\operatorname{orb}\left(\tau, \Gamma_{\tau}\right)$ is does not intersect the affine invariant open set $T^{\Gamma_{\sigma}}$; if $\tau \leq \sigma$, for $\sigma \in \Sigma$ the closure of the orbit $\operatorname{orb}\left(\tau, \Gamma_{\tau}\right)$ in the affine open set $T^{\Gamma_{\sigma}}$ is equal to $T^{\Gamma_{\sigma} \cap \tau^{\perp}}$ (see Lemma 3.6).

If $\tau \leq \theta \leq \sigma$ then $\theta(\tau) \leq \sigma(\tau)$ and $\theta^{\perp} \subset \tau^{\perp}$ hence $M\left(\theta, \Gamma_{\sigma}\right)=M\left(\theta(\tau), \Gamma_{\sigma} \cap \tau^{\perp}\right)$ is the sublattice spanned by $\Gamma_{\sigma} \cap \theta^{\perp}$.

If $\tau \leq \sigma, \sigma^{\prime}$ and if $\theta=\sigma \cap \sigma^{\prime}$ then we deduce from condition ii. in Definition 4.1 that:

$$
\Gamma_{\theta} \cap \tau^{\perp}=\Gamma_{\sigma} \cap \tau^{\perp}+M\left(\theta(\tau), \Gamma_{\sigma(\tau)}\right)=\Gamma_{\sigma^{\prime}} \cap \tau^{\perp}+M\left(\theta(\tau), \Gamma_{\sigma^{\prime}(\tau)}\right) .
$$

We obtain that the triple $\left(N\left(\tau, \Gamma_{\tau}\right), \Sigma(\tau), \Gamma(\tau)\right)$ satisfies the axioms in Definition 4.1 with respect to the torus $T^{M\left(\tau, \Gamma_{\tau}\right)}$.

We have also described an embedding $T_{\Sigma(\tau)}^{\Gamma(\tau)} \hookrightarrow T_{\Sigma}^{\Gamma}$ in such a way that the intersection of this variety with any affine chart containing $\operatorname{orb}(\tau, \Gamma)$ is the closure of the orbit $\operatorname{orb}(\tau, \Gamma)$ in the chart. The conclusion follows from Lemma 4.8.

Remark 4.11. The non-singular locus of the toric variety $T_{\Sigma}^{\Gamma}$ is the union of the orbits $\operatorname{orb}(\tau, \Gamma)$ corresponding to regular cones $\tau \in \Sigma$ such their index $i\left(\tau, \Gamma_{\tau}\right)$ is equal to 1 .

\section{Blowing UpS}

The theory of normal toric varieties deals with normalized equivariant blowing ups, i.e., blowing ups of equivariant ideals followed by normalization. In this section we build blowing ups of equivariant ideals in toric varieties.

Let $\sigma$ be a strictly convex rational cone in $N_{\mathbf{R}}$ and $\Gamma$ a subsemigroup of finite type of the lattice $M$ such that $\mathbf{Z} \Gamma=M$ and the saturation of $\Gamma$ in $M$ is equal to $\check{\sigma} \cap M$. For simplicity we assume that the cone $\sigma$ is of dimension $d$ hence $\check{\sigma}$ is strictly convex.

Let us consider a graded ideal $\mathcal{I}$ in $A\left[t^{\Gamma}\right]$, which is necessarily generated by monomials $t^{m_{1}}, \ldots, t^{m_{k}}$. We build the corresponding Newton polyhedron $\mathcal{N}_{\sigma}(\mathcal{I})$, by definition the convex hull in $M_{\mathbf{R}}$ of the $m_{i}+\check{\sigma}$, which is also the convex hull of the set $|\mathcal{I}|$ of exponents 
of monomials belonging to the ideal $\mathcal{I}$ of $A\left[t^{\Gamma}\right]$. It is quite convenient to denote with the same letter $\mathcal{I}$ the set $\left\{m_{1}, \ldots m_{k}\right\}$.

The set $\mathcal{I}$ determines the order function:

$$
\operatorname{ord}_{\mathcal{I}}: \sigma \rightarrow \mathbf{R}, \quad \nu \mapsto \min _{m \in \mathcal{I}}\langle\nu, m\rangle .
$$

The order function $\operatorname{ord}_{\mathcal{I}}$ coincides with the support function $\grave{a}$ la Minkowski of the polyhedron $\mathcal{N}=\mathcal{N}_{\sigma}(\mathcal{I})$ defined as the function $H: N_{\mathbf{R}} \rightarrow \mathbf{R}$ given by $H(\nu)=\min _{m \in \mathcal{N}}\langle\nu, m\rangle$. It is a gauge $\left(\operatorname{ord}_{\mathcal{I}}(\lambda u)=\lambda \operatorname{ord}_{\mathcal{I}}(u)\right.$ for $\left.\lambda>0\right)$ which is piecewise linear. The maximal cones of linearity of the function $\operatorname{ord}_{\mathcal{I}}$ form the $d$-skeleton of the fan $\Sigma(\mathcal{I})$ subdividing $\sigma$. Each such cone $\sigma_{i}$ in the $d$-skeleton of $\Sigma(\mathcal{I})$ is the convex dual of the convex rational cone generated by the vectors $\left(m-m_{i}\right)_{m \in \mathcal{N}_{\sigma}(\mathcal{I})}$, where $m_{i}$ is a vertex of $\mathcal{N}_{\sigma}(\mathcal{I})$. The correspondence $m_{i} \mapsto \sigma_{i}$ is a bijection between the set of vertices $\left\{m_{1}, \ldots, m_{s}\right\} \subset \mathcal{I}$ of the polyhedron $\mathcal{N}_{\sigma}(\mathcal{I})$ and the $d$-skeleton of $\Sigma(\mathcal{I})$, such that

$$
m_{i} \mapsto \sigma_{i} \text { if and only if } \operatorname{ord}_{\mathcal{I}}(\nu)=\left\langle\nu, m_{i}\right\rangle \text { for all } \nu \in \sigma_{i} \text {. }
$$

Note that $\Gamma \subset \check{\sigma} \cap M \subset \check{\sigma}_{i} \cap M$. In each of the cones $\check{\sigma}_{i}$ we consider the semigroup

$$
\Gamma_{i}=\Gamma+\left\langle m_{1}-m_{i}, \ldots, m_{i-1}-m_{i}, m_{i+1}-m_{i}, \ldots, m_{k}-m_{i}\right\rangle \subset \check{\sigma}_{i} \cap M .
$$

By Lemma 1.3, the saturation in $M$ of this semigroup is equal to $\check{\sigma}_{i} \cap M$. We denote by $\Gamma(\mathcal{I})$ the set consisting of the semigroups $\Gamma_{i}$, together with $\Gamma_{i, \tau}$ (defined by equation (2)) for $\tau \leq \sigma_{i}, i=1, \ldots, s$.

Proposition 5.1. The triple $(N, \Sigma(\mathcal{I}), \Gamma(\mathcal{I}))$ defines a toric scheme $B$ over Spec $A$. The inclusions $\Gamma \subset \Gamma_{i}, i=1, \ldots, s$, determine a map of schemes

$$
\pi: B \rightarrow \operatorname{Spec} A\left[t^{\Gamma}\right]
$$

over Spec A, which is the blowing up of the ideal $\mathcal{I}$.

Proof. We prove first that the triple $(N, \Sigma(\mathcal{I}), \Gamma(\mathcal{I}))$ satisfies the compatibility conditions stated in Definition 4.1. By Lemma 3.9 it is enough to check them for the affine open sets corresponding to two vertices, say $m_{1}$ and $m_{2}$, of $\mathcal{N}_{\sigma}(\mathcal{I})$. Then, if $\tau=\sigma_{1} \cap \sigma_{2}$ the condition we have to prove is that $\Gamma_{1, \tau}=\Gamma_{2, \tau}$.

Notice that the vector $m:=m_{2}-m_{1} \in \Gamma_{1}$ belongs to the interior of $\check{\sigma}_{1} \cap \tau^{\perp}$. By Lemma 3.9 and the definitions we get $\Gamma_{1, \tau}=\Gamma_{1}+\mathbf{Z}_{\geq 0}(-m)$ and similarly $\Gamma_{2, \tau}=$ $\Gamma_{2}+\mathbf{Z}_{\geq 0} m$. Then the assertion follows since $\Gamma_{1, \tau}$, which is equal to

$$
\Gamma+\mathbf{Z}\left(m_{2}-m_{1}\right)+\sum_{j=2, \ldots, k} \mathbf{Z}_{\geq 0}\left(m_{j}-m_{1}\right)=\Gamma+\mathbf{Z}\left(m_{1}-m_{2}\right)+\sum_{j=2, \ldots, k} \mathbf{Z}_{\geq 0}\left(m_{j}-m_{2}\right),
$$

is the same semigroup as $\Gamma_{2, \tau}$.

It follows that the scheme $B$ is covered by the affine sets Spec $A\left[t^{\Gamma_{i}}\right]$ for $i=1, \ldots, s$. Since each $\Gamma_{i}$ contains $\Gamma$, there is a natural map $\pi$ : Spec $A\left[t^{\Gamma_{i}}\right] \rightarrow \operatorname{Spec} A\left[t^{\Gamma}\right]$. The sheaf of ideals on $B$ determined by the compositions with $\pi$ of the generators of $\mathcal{I}$ is generated by $t^{m_{i}} \circ \pi$ in the chart $\operatorname{Spec} A\left[t^{\Gamma_{i}}\right]$.

It is not difficult to prove that any semigroup $\Gamma_{i}$ defined by (44), for $i>s$, that is when $m_{i}$ is not a vertex of $\mathcal{N}_{\sigma}(\mathcal{I})$, is of the form $\Gamma_{j, \tau}$ for some $1 \leq j \leq s$ and $\tau \leq \sigma_{j}$. This means that the corresponding affine chart Spec $A\left[t^{\Gamma_{i}}\right]$ of the blowing up of $\mathcal{I}$ is in fact an affine open subset of Spec $A\left[t^{\Gamma_{j}}\right]$, where $m_{j}$ is a vertex of $\mathcal{N}_{\sigma}(\mathcal{I})$.

Remark 5.2. With the above notations let us consider the Rees algebra of $\mathcal{I}$, defined as $R[\mathcal{I}]=\bigoplus_{l \geq 0} \mathcal{I}^{l} s^{l}$. Since each power $\mathcal{I}^{l}$ is a monomial ideal, the term $\mathcal{I}^{l} s^{l}$ is of the form $\mathcal{I}^{l} s^{l}=\oplus_{\gamma \in\left|\mathcal{I}^{l}\right|} t^{\gamma} s^{l}$. Consider also the semigroup $\Gamma_{\mathcal{I}}$ of $M \times \mathbf{Z}$ generated by $(\Gamma \times\{0\}) \cup(|\mathcal{I}| \times\{1\})$. By using the map of semigroups $\Gamma \rightarrow \Gamma_{\mathcal{I}}$, defined by $\gamma \mapsto(\gamma, 0)$, the semigroup algebra $A\left[t^{\Gamma_{\mathcal{I}}}\right]$ has the structure of $A\left[t^{\Gamma}\right]$-algebra. There is a unique 
isomorphism of semigroups algebras $R[\mathcal{I}] \rightarrow A\left[t^{\Gamma_{\mathcal{I}}}\right]$ over $A$, such that $t^{\gamma} s^{l} \mapsto t^{(\gamma, l)}$. This is also an isomorphism of $A\left[t^{\Gamma}\right]$ - graded algebras, when the grading of a monomial $t^{(\gamma, l)}$ is defined to be equal to $l$. The canonical map $\operatorname{Proj}(R[\mathcal{I}]) \rightarrow \operatorname{Spec} A\left[t^{\Gamma}\right]$ is the blowing up of the ideal $\mathcal{I}$. We have given in Proposition 5.1 the combinatorial description of this map as a toric morphism as defined in Section 6. See also Section 11.3 [5].

Corollary 5.3. The blowing-up of an equivariant sheaf of ideals on a toric variety $T_{\Sigma}^{\Gamma}$ is a toric variety. Its description above each equivariant open affine chart of $T_{\Sigma}^{\Gamma}$ is given by Proposition 5.1 .

\section{TORIC MORPHISMS}

Recall that a morphism $\phi: T^{M^{\prime}} \rightarrow T^{M}$ of algebraic tori gives rise to two group homomorphisms

$$
\phi^{*}: M \rightarrow M^{\prime} \text { and } \phi_{*}: N^{\prime} \rightarrow N
$$

between the corresponding lattices of characters and between the corresponding lattices of one-parameter subgroups. The homomorphisms $\phi^{*}$ and $\phi_{*}$ are mutually dual and determine the morphism $\phi: T^{M^{\prime}} \rightarrow T^{M}$ of algebraic tori. Note that $\phi$ is defined algebraically by

$$
k\left[t^{M}\right] \rightarrow k\left[t^{M^{\prime}}\right], \quad t^{m} \mapsto t^{\phi^{*}(m)}, \quad m \in M .
$$

Now suppose that we have two toric varieties $T_{\Sigma}^{\Gamma}$ and $T_{\Sigma^{\prime}}^{\Gamma^{\prime}}$ with respective tori $T^{M}$ and $T^{M^{\prime}}$ defined by the combinatorial data given by the triples $(N, \Sigma, \Gamma)$ and $\left(N^{\prime}, \Sigma^{\prime}, \Gamma^{\prime}\right)$ (see Definition 4.1).

Definition 6.1. The homomorphism $\phi_{*}$ is a map of fans with attached semigroups $(N, \Sigma, \Gamma) \rightarrow\left(N^{\prime}, \Sigma^{\prime}, \Gamma^{\prime}\right)$ if for any $\sigma^{\prime} \in \Sigma^{\prime}$ there exists $\sigma \in \Sigma$ such that $\phi^{*}\left(\Gamma_{\sigma}\right) \subset \Gamma_{\sigma^{\prime}}^{\prime}$.

Note then that $\phi_{*}$ is a map of fans, that is, for any $\sigma^{\prime} \in \Sigma^{\prime}$ there is a cone $\sigma \in \Sigma$ such that image by $\sigma^{\prime}$ by the R-linear extension of $\phi_{*}$ is contained in $\sigma$. See Section 1.5 [26].

Proposition 6.2. Let $\phi: T^{M^{\prime}} \rightarrow T^{M}$ be a morphism of algebraic tori. If $\phi_{*}$ defines a map of fans with attached semigroups $(N, \Sigma, \Gamma) \rightarrow\left(N^{\prime}, \Sigma^{\prime}, \Gamma^{\prime}\right)$ then it gives rise to a morphism: $\bar{\phi}: T_{\Sigma^{\prime}}^{\Gamma^{\prime}} \rightarrow T_{\Sigma}^{\Gamma}$ which extends $\phi: T^{M^{\prime}} \rightarrow T^{M}$ and is equivariant with respect to $\phi$. Conversely, if $f: T_{\Sigma^{\prime}}^{\Gamma^{\prime}} \rightarrow T_{\Sigma}^{\Gamma}$ is an equivariant morphism with respect to $\phi$ then $\phi_{*}$ defines a map of fans with attached semigroups $\left(N^{\prime}, \Sigma^{\prime}, \Gamma^{\prime}\right) \rightarrow(N, \Sigma, \Gamma)$ and $f=\bar{\phi}$. In addition we have a commutative diagram

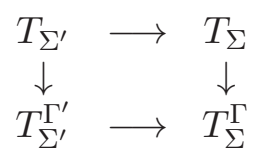

where the vertical arrows are normalizations and the horizontal ones are the toric morphisms which extend $\phi: T^{M^{\prime}} \rightarrow T^{M}$.

Proof. For any $\sigma^{\prime} \in \Sigma^{\prime}$ there exists a cone $\sigma \in \Sigma$ such that the restriction of $\phi^{*}$ determines a semigroup homomorphism $\Gamma_{\sigma} \rightarrow \Gamma_{\sigma^{\prime}}^{\prime}$. The corresponding homomorphism of $k$-algebras $k\left[\Gamma_{\sigma}\right] \rightarrow k\left[\Gamma_{\sigma^{\prime}}^{\prime}\right]$ defines a morphism:

$$
\bar{\phi}_{\sigma^{\prime}, \sigma}: T^{\Gamma_{\sigma^{\prime}}^{\prime}} \rightarrow T^{\Gamma_{\sigma}} \text { given on closed points by } \bar{\phi}_{\sigma^{\prime}, \sigma}(x)=x \circ \phi_{\mid \Gamma_{\sigma}}^{*},
$$

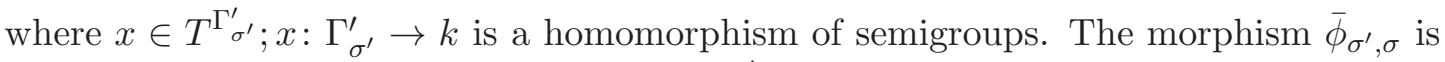
equivariant through $\phi$ since for any $y \in T^{M^{\prime}}, y: M^{\prime} \rightarrow k^{*}$ group homomorphism and any $x \in T^{\Gamma^{\prime}{ }_{\sigma^{\prime}}}$ we get:

$$
\bar{\phi}_{\sigma^{\prime}, \sigma}(y \cdot x)=(y \cdot x) \circ \phi_{\mid \Gamma_{\sigma}}^{*}=\left(y \circ \phi^{*}\right) \cdot\left(x \circ \phi_{\mid \Gamma_{\sigma}}^{*}\right)=\phi(y) \cdot \bar{\phi}_{\sigma^{\prime}, \sigma}(x) .
$$


By gluing-up the affine pieces together we get a morphism $\bar{\phi}: T_{\Sigma^{\prime}}^{\Gamma^{\prime}} \rightarrow T_{\Sigma}^{\Gamma}$ which is equivariant with respect to $\phi$.

For the converse, since $f$ is assumed to be equivariant through $\phi$ the image by $f$ of each orbit of the action of $T^{M^{\prime}}$ on $T_{\Gamma^{\prime}}^{\Sigma^{\prime}}$ is contained in one orbit of the action of $T^{M}$ on $T_{\Sigma}^{\Gamma}$. If $\tau^{\prime} \leq \sigma^{\prime}$ and $\sigma^{\prime} \in \Sigma^{\prime}$ then the orbit $\operatorname{orb}\left(\sigma^{\prime}, \Gamma_{\sigma^{\prime}}^{\prime}\right)$ is contained in the closure of $\operatorname{orb}\left(\tau^{\prime}, \Gamma_{\tau^{\prime}}^{\prime}\right)$ by Proposition 3.6. Then there exist $\sigma, \tau \in \Sigma$ such that

$$
f\left(\operatorname{orb}\left(\sigma^{\prime}, \Gamma_{\sigma^{\prime}}^{\prime}\right)\right) \subset \operatorname{orb}\left(\sigma, \Gamma_{\sigma}\right) \text { and } f\left(\operatorname{orb}\left(\tau^{\prime}, \Gamma_{\tau^{\prime}}^{\prime}\right)\right) \subset \operatorname{orb}\left(\tau, \Gamma_{\tau}\right) .
$$

Since $f$ is continuous $\operatorname{orb}\left(\sigma, \Gamma_{\sigma}\right)$ must be contained in the closure of $\operatorname{orb}\left(\tau, \Gamma_{\tau}\right)$, hence $\tau$ is a face of $\sigma$ by Proposition 3.6 and Lemma 3.5. By (11) it follows that $f\left(T^{\Gamma_{\sigma^{\prime}}^{\prime}}\right) \subset T^{\Gamma_{\sigma}}$. The restriction $f_{\mid T^{\Gamma^{\prime} \sigma^{\prime}}}: T^{\Gamma_{\sigma^{\prime}}^{\prime}} \rightarrow T^{\Gamma_{\sigma}}$ is equivariant with respect to $\phi: T^{M^{\prime}} \rightarrow T^{M}$. Hence $f_{\mid T^{\Gamma^{\prime}}{ }^{\prime}}: T^{\Gamma_{\sigma^{\prime}}^{\prime}} \rightarrow T^{\Gamma_{\sigma}}$ is defined algebraically by the homomorphism of $k$-algebras $k\left[t^{\Gamma_{\sigma}}\right] \rightarrow k\left[t^{\Gamma_{\sigma^{\prime}}^{\prime}}\right]$, which is obtained by restriction from the homomorphism of $k$-algebras $k\left[t^{M}\right] \rightarrow k\left[t^{M^{\prime}}\right]$ which maps $t^{m} \mapsto t^{\phi^{*}(m)}$ for $m \in M$. This implies that $\phi^{*}\left(\Gamma_{\sigma}\right) \subset \Gamma_{\sigma^{\prime}}^{\prime}$ and also that $f=\bar{\phi}$.

Since $\phi_{*}$ is a map of fans it defines a toric morphism between the normalizations of $T_{\Sigma^{\prime}}^{\Gamma^{\prime}}$ and $T_{\Sigma}^{\Gamma}$. Finally, it is easy to check that the diagram above is commutative.

It is sometimes useful to consider morphisms of toric varieties which send the torus of the source into a non dense orbit of the target: Let $(N, \Sigma, \Gamma)$ and $\left(N^{\prime}, \Sigma^{\prime}, \Gamma^{\prime}\right)$ be two triples defining toric varieties $T_{\Sigma}^{\Gamma}$ and $T_{\Sigma^{\prime}}^{\Gamma^{\prime}}$. Let $\tau$ be a cone of $\Sigma$. Suppose that we have a morphism of algebraic tori $\phi: T^{M^{\prime}} \rightarrow T^{M\left(\tau, \Gamma_{\tau}\right)}$ such that $\phi_{*}: N^{\prime} \rightarrow N\left(\tau, \Gamma_{\tau}\right)$ defines a map of fans with attached semigroups $\left(N^{\prime}, \Sigma^{\prime}, \Gamma^{\prime}\right) \rightarrow\left(N\left(\tau, \Gamma_{\tau}\right), \Sigma(\tau), \Gamma(\tau)\right)$. Then by Proposition 6.2 and Lemma 3.5 we have a toric morphism

$$
\bar{\phi}: T_{\Sigma^{\prime}}^{\Gamma^{\prime}} \rightarrow T_{\Sigma(\tau)}^{\Gamma(\tau)} .
$$

Let us denote by $n: T_{\Sigma} \rightarrow T_{\Sigma}^{\Gamma}$ the normalization map and by $\bar{i}_{\tau}: T_{\Sigma(\tau)} \rightarrow T_{\Sigma}$ the closed embedding of the closure of $\operatorname{orb}(\tau)$ in $T_{\Sigma}$. The following Proposition is consequence of Proposition 6.2 and Lemma 3.5

Proposition 6.3. The composite of $\bar{\phi}$ with the closed embedding $i_{\tau}: T_{\Sigma(\tau)}^{\Gamma(\tau)} \hookrightarrow T_{\Sigma}^{\Gamma}$ lifts to the normalization of $T_{\Sigma}^{\Gamma}$, i.e., there exists a toric morphism $\psi: T_{\Sigma^{\prime}}^{\Gamma^{\prime}} \rightarrow T_{\Sigma(\tau)}$ such that $i_{\tau} \circ \bar{\phi}=n \circ \bar{i}_{\tau} \circ \psi$ if and only if there is a lattice homomorphism $\varphi^{*}: M(\tau) \rightarrow M^{\prime}$ such that $\varphi_{\mid M\left(\tau, \Gamma_{\tau}\right)}^{*}=\phi^{*}$ and then $\psi=\bar{\varphi}$.

Example 6.4. By Proposition 6.3 the map $u \mapsto(u, 0,0)$, which parametrizes the singular locus of the Whitney umbrella $\left\{x_{1}^{2} x_{2}-x_{3}^{2}=0\right\}$ does not lift to the normalization while $u \mapsto\left(u^{2}, 0,0\right)$ does.

\section{Abstract toric varieties}

We recall the usual definition of toric variety.

Definition 7.1. A toric variety $X$ is an irreducible (separated) algebraic variety equipped with an action of an algebraic torus $T$ embedded in $X$ as a Zariski open set such that the action of $T$ on $X$ is morphism which extends the action of $T$ over itself by multiplication.

As stated in Proposition 3.7 any affine toric variety is the spectrum of certain semigroup algebra. Gel'fand, Kapranov, and Zelevinsky have defined and studied those projective toric varieties which are equivariantly embedded in the projective space, which is viewed as a toric variety, see [12], Chapter 5 . 
The following Theorem, which is consequence of a more general result of Sumihiro, provides the key to establish a combinatorial description of normal toric varieties.

Theorem 7.2. (see [32]) Any normal toric variety $X$ has a finite covering by $T$ invariant affine normal toric varieties.

The statement of Theorem 7.2 does not hold if the normality assumption is dropped.

Example 7.3. Let $C \subset \mathbf{P}_{\mathbf{C}}^{2}$ be the projective nodal cubic with equation $y^{2} z-x^{2}(x+z)$. It is a rational curve with a node singularity at $P=(0: 0: 1)$ and only one point $Q=(0: 1: 0)$ at the line of infinity $z=0$. The curve $C$ is rational and has a parametrization $\pi: \mathbf{P}_{\mathbf{C}}^{1} \rightarrow C$ such that $\pi(0)=\pi(\infty)=P$ and $\pi(1)=Q$. Then we have that $\pi_{\mid \mathbf{C}^{*}}: \mathbf{C}^{*} \rightarrow C \backslash\{P\}$ is an isomorphism. The multiplicative action of $\mathbf{C}^{*}$ on $\mathbf{P}_{\mathbf{C}}^{1}$ corresponds by $\pi$ to the group law action on the cubic hence it is algebraic. It follows that $C$ is a toric variety with respect to Definition 7.1 . Notice that $C$ is the only open set containing $P$ which is invariant by the action of $\mathbf{C}^{*}$. This example is also a projective toric curve which does not admit any equivariant embedding in the projective space (see 25] page 4 and [12] Chapter 5, Remark 1.6).

Definition 7.4. An action of a group on an algebraic variety $X$ is good if $X$ is covered by a finite number of affine open subsets which are invariant by the action.

We modify the abstract definition of toric varieties as follows:

Definition 7.5. A toric variety $X$ is an irreducible separated algebraic variety equipped with a good action of an algebraic torus $T$ embedded in $X$ as a Zariski open set such that the action of $T$ on $X$ extends the action of $T$ on itself by multiplication.

Theorem 7.6. If $X$ is a toric variety in the sense of Definition 7.5 with torus $T$, then there exists a triple $(N, \Sigma, \Gamma)$ as in Definition 4.1 and an isomorphism $\varphi: T \rightarrow T^{M}$ such that the pair $(T, X)$ is equivariantly isomorphic to $\left(T^{M}, T_{\Sigma}^{\Gamma}\right)$ with respect to $\varphi$.

Proof. We denote by $M$ the lattice of characters of the torus $T$ hence $T=T^{M}$ and $N$ is the dual lattice of $M$.

By Proposition 3.7 an affine $T^{M}$-invariant open subset is of the form $T^{\Gamma_{\sigma}}$ where $\Gamma_{\sigma}$ is a subsemigroup of finite type of $M$ such that $\mathbf{Z} \Gamma_{\sigma}=M$, and $\sigma \subset N_{\mathbf{R}}$ is the dual cone of $\check{\sigma}=\mathbf{R}_{\geq 0} \Gamma_{\sigma} \subset M_{\mathbf{R}}$. By Lemma 3.10 the open affine $T^{M}$-invariant subsets of $T^{\Gamma_{\sigma}}$ are $T^{\Gamma_{\tau}}$, for $\tau \leq \sigma$, where $\Gamma_{\tau}=\Gamma_{\sigma}+M\left(\tau, \Gamma_{\sigma}\right)$.

By definition $X$ is covered by a finite number of $T^{M}$-invariant affine open subsets of the form $\left\{T^{\Gamma_{\sigma}}\right\}_{\sigma \in \Sigma}$. We can assume that if $\sigma \in \Sigma$ and if $\tau \leq \sigma$ then $\tau \in \Sigma$. We are going to show that $\Sigma$ is a fan in $N_{\mathbf{R}}$, hence $T^{\Gamma_{\sigma}} \neq T^{\Gamma_{\sigma^{\prime}}}$ if $\sigma \neq \sigma^{\prime}$.

We have that for any $\sigma, \sigma^{\prime} \in \Sigma$ the intersection $T^{\Gamma_{\sigma}} \cap T^{\Gamma_{\sigma^{\prime}}}$ is an affine open subset of the separated variety $X$ (see Chapter 2 of [18]). It is also a $T^{M}$-invariant affine subset of both $T^{\Gamma_{\sigma}}$ and $T^{\Gamma_{\sigma^{\prime}}}$, hence it is of the form $T^{\Gamma_{\tau}}$. By Lemma 3.10 we obtain two inclusion of semigroups $\Gamma_{\sigma} \rightarrow \Gamma_{\tau}$ and $\Gamma_{\sigma^{\prime}} \rightarrow \Gamma_{\tau}$. Since $X$ is separated the diagonal map $T^{\Gamma_{\tau}} \rightarrow T^{\Gamma_{\sigma}} \times T^{\Gamma_{\sigma^{\prime}}}$ is a closed embedding (see Chapter 2 of [18]). Algebraically, this implies the surjectivity of the homomorphism

$$
k\left[t^{\Gamma_{\sigma}}\right] \otimes_{k} k\left[t^{\Gamma_{\sigma^{\prime}}}\right] \rightarrow k\left[t^{\Gamma_{\tau}}\right] \text {, determined by } t^{\gamma} \otimes t^{\gamma^{\prime}} \mapsto t^{\gamma+\gamma^{\prime}} .
$$

It follows that the homomorphism of semigroups $\Gamma_{\sigma} \times \Gamma_{\sigma^{\prime}} \rightarrow \Gamma_{\tau},\left(\gamma, \gamma^{\prime}\right) \mapsto \gamma+\gamma^{\prime}$ is surjective. This proves that $\Gamma_{\tau}=\Gamma_{\sigma}+\Gamma_{\sigma^{\prime}}$ thus

$$
\mathbf{R}_{\geq 0} \Gamma_{\tau}=\check{\tau}=\mathbf{R}_{\geq 0}\left(\Gamma_{\sigma}+\Gamma_{\sigma^{\prime}}\right)=\check{\sigma}+\check{\sigma^{\prime}}
$$

By duality we deduce that $\tau=\sigma \cap \sigma^{\prime}$. By Proposition 3.10 we obtain

$$
\Gamma_{\tau}=\Gamma_{\sigma}+M\left(\tau, \Gamma_{\sigma}\right)=\Gamma_{\sigma^{\prime}}+M\left(\tau, \Gamma_{\sigma^{\prime}}\right) .
$$


In conclusion, $\Sigma$ is a fan in $N_{\mathbf{R}}$ and if $\Gamma:=\left\{\Gamma_{\sigma} \mid \sigma \in \Sigma\right\}$ the triple $(N, \Sigma, \Gamma)$ satisfies the compatibility properties of Definition 4.1 and the variety $T_{\Sigma}^{\Gamma}$ is $T^{M}$-equivariantly isomorphic to $X$.

The following corollary is consequence of Proposition 6.2 and Theorem 7.6.

Corollary 7.7. The category whose objects are the triples $(N, \Sigma, \Gamma)$ of Definition 4.1 and whose morphisms are the maps of fans with attached semigroups of Definition 6.1 is equivalent to the category whose objects are the toric varieties of Definition 7.5 and whose morphisms are those equivariant morphism which extend morphisms of the corresponding algebraic tori; see Proposition 6.2.

\section{INVERTIBLE SHEAVES ON TORIC VARIETIES}

In this section we describe how some of the classical results in the study of equivariant invertible sheaves on a normal toric variety extend to the general case.

Let $T_{\Sigma}^{\Gamma}$ denote a toric variety defined by the triple $(N, \Sigma, \Gamma)$. Recall that if $\sigma \in \Sigma$ we denote by $T_{\sigma}=T^{\check{\sigma} \cap M}$ the normalization of the chart $T^{\Gamma_{\sigma}}$ and by $T_{\Sigma}$ the normalization of $T_{\Sigma}^{\Gamma}$.

A support function $h:|\Sigma| \rightarrow \mathbf{R}$ is a continuous function such that for each $\sigma \in \Sigma$ the restriction $h_{\mid \sigma}: \sigma \rightarrow \mathbf{R}$ is linear. We say that $h$ is integral with respect to $N$ if $h(|\Sigma| \cap N) \subset \mathbf{Z}$. We denote by $\operatorname{SF}(N, \Sigma)$ the set of support functions integral with respect to $N$. If $h$ is a support function integral with respect to $N$ then for any $\sigma \in \Sigma$ there exists $m_{\sigma} \in M$ such that

$$
h(\nu)=\left\langle\nu, m_{\sigma}\right\rangle, \quad \text { for all } \nu \in \sigma .
$$

Notice that by continuity we have that

$$
m_{\tau}=m_{\sigma} \bmod M(\tau)=M \cap \tau^{\perp}, \text { for } \tau \leq \sigma, \sigma \in \Sigma .
$$

The set $\left\{m_{\sigma} \mid \sigma \in \Sigma\right\}$ determines $h$ but may not be uniquely determined.

Definition 8.1. A support function for the triple $(N, \Sigma, \Gamma)$ is a support function $h$ : $|\Sigma| \rightarrow \mathbf{R}$ integral with respect to $N$ which in addition has the compatibility property

$$
m_{\tau}=m_{\sigma} \bmod M\left(\tau, \Gamma_{\tau}\right), \text { for } \tau \leq \sigma, \sigma \in \Sigma .
$$

We denote by $\operatorname{SF}(N, \Sigma, \Gamma)$ the additive group of support functions for the triple $(N, \Sigma, \Gamma)$. It is a subgroup of $\operatorname{SF}(N, \Sigma)$. A vector $m \in M$ defines an element of $\mathrm{SF}(N, \Sigma, \Gamma)$ hence we have a homomorphism $M \rightarrow \mathrm{SF}(N, \Sigma, \Gamma)$, which is injective if the support of $\Sigma$ spans $N_{\mathbf{R}}$ as a real vector space.

Any $h \in \mathrm{SF}(N, \Sigma)$ determines $T^{M}$-invariant Cartier divisor $D_{h}$ on $T_{\Sigma}$ by

$$
D_{h \mid T_{\sigma}}=\operatorname{div}\left(t^{-m_{\sigma}}\right) \text { for } \sigma \in \Sigma,
$$

where $\operatorname{div}(g)$ denotes the principal Cartier divisor of the rational function $g$ on an irreducible variety. Notice that $D_{h}$ is independent of the possible choices of different Cartier data $\left\{m_{\sigma} \mid \sigma \in \Sigma\right\}$ defining $h$. If $\sigma, \sigma^{\prime} \in \Sigma, \tau=\sigma \cap \sigma^{\prime}$ then $T_{\tau}=T_{\sigma} \cap T_{\sigma^{\prime}}$ and (5) guarantees that $t^{-m_{\sigma}+m_{\sigma^{\prime}}}$ and $t^{m_{\sigma}-m_{\sigma^{\prime}}}$ are both regular functions on $T_{\tau}$. Any $T^{M}$-invariant Cartier divisor on $T_{\Sigma}$ is of the form $D_{h}$ for $h \in \operatorname{SF}(N, \Sigma)$, i.e., it is defined by Cartier data.

Lemma 8.2. If $h \in S F(N, \Sigma)$ is defined by the Cartier data $\left\{m_{\sigma} \mid \sigma \in \Sigma\right\}$ then it defines a $T^{M}$-invariant Cartier divisor on $T_{\Sigma}^{\Gamma}$ if and only if (6) holds, that is, if and only if $h \in S F(N, \Sigma, \Gamma)$. 
Proof. The condition to determine a Cartier divisor is that for any $\sigma, \sigma^{\prime} \in \Sigma, \tau=\sigma \cap \sigma^{\prime}$ the transition function $t^{-m_{\sigma}+m_{\sigma^{\prime}}}$ is an invertible regular function on $T^{\Gamma_{\tau}}=T^{\Gamma_{\sigma}} \cap T^{\Gamma_{\sigma^{\prime}}}$. By Lemma 3.9 this is equivalent to (6) .

We have shown that the group $\operatorname{CDiv}_{T^{M}}\left(T_{\Sigma}^{\Gamma}\right)$ of $T^{M}$-invariant Cartier divisors on $T_{\Sigma}^{\Gamma}$ can be seen as a subset of $\operatorname{CDiv}_{T^{M}}\left(T_{\Sigma}\right)$. The set $\left\{\operatorname{div}\left(t^{m}\right)\right\}_{m \in M}$ is a subgroup of $\mathrm{CDiv}_{T^{M}}\left(T_{\Sigma}^{\Gamma}\right)$ consisting of principal Cartier divisors.

The map

$$
\mathrm{SF}(N, \Sigma, \Gamma) \longrightarrow \operatorname{CDiv}_{T^{M}}\left(T_{\Sigma}^{\Gamma}\right), \quad h \mapsto D_{h} .
$$

is a group isomorphism. The inverse map sends a Cartier divisor $D$ on $T_{\Sigma}^{\Gamma}$, given by the Cartier data $\left\{m_{\sigma} \mid \sigma \in \Sigma\right\}$, to the function

$$
h_{D}:=|\Sigma| \rightarrow \mathbf{R}, \quad h_{D}(\nu)=\left\langle\nu, m_{\sigma}\right\rangle \text { if } \nu \in \sigma .
$$

A Cartier divisor on $T_{\Sigma}$ determines an invertible sheaf $\mathcal{O}_{T_{\Sigma}}(D)$. If $U$ is an affine open set in which $D=\operatorname{div}\left(g_{U}\right)$ for some rational function $g_{U}$ then the set of sections $H^{0}\left(U, \mathcal{O}_{T_{\Sigma}}(D)\right)$ consists of those rational functions $f$ such that $f g_{U}$ is a regular function on $U$.

We denote by $\mathcal{O}_{T_{\Sigma}^{\Gamma}}$ the structure sheaf on the toric variety $T_{\Sigma}^{\Gamma}$. The invertible sheaf of a $T^{M}$-invariant Cartier divisor $D$ on $T_{\Sigma}^{\Gamma}$ is the sheaf of $\mathcal{O}_{T_{\Sigma}^{\Gamma}}$-modules $\mathcal{O}_{T_{\Sigma}^{\Gamma}}(D)$. By (77) the set of sections of this sheaf on $T^{\Gamma_{\sigma}}$ is

$$
H^{0}\left(T^{\Gamma_{\sigma}}, \mathcal{O}_{T_{\Sigma}^{\Gamma}}(D)\right)=t^{m_{\sigma}} k\left[t^{\Gamma_{\sigma}}\right] .
$$

We denote by $P_{D}^{\Gamma}$ the following subset of $M$ :

$$
P_{D}^{\Gamma}:=\bigcap_{\sigma \in \Sigma} m_{\sigma}+\Gamma_{\sigma}
$$

The set of global sections of the sheaf $\mathcal{O}_{T_{\Sigma}^{\Gamma}}(D)$ is equal to

$$
H^{0}\left(T_{\Sigma}^{\Gamma}, \mathcal{O}_{T_{\Sigma}^{\Gamma}}(D)\right)=\bigcap_{\sigma \in \Sigma} t^{m_{\sigma}} k\left[t^{\Gamma_{\sigma}}\right]=\bigoplus_{m \in P_{D}^{\Gamma}} k t^{m} .
$$

Remark 8.3. As in the normal case, a $T^{M}$-invariant Cartier divisor $D$ defines an equivariant line bundle $\mathcal{L}_{D}$ whose sections coincide with those of the invertible sheaf $\mathcal{O}_{T_{\Sigma}^{\Gamma}}(D)$. See [26], Chapter 2.

The Picard group $\operatorname{Pic}(X)$ of a variety $X$ consists of the isomorphism classes of invertible sheaves in $X$.

Lemma 8.4. Suppose that $|\Sigma|=N_{\mathbf{R}}$. For any Cartier divisor $D$ on the toric variety $T_{\Sigma}^{\Gamma}$ we have an $\mathcal{O}_{T_{\Sigma}^{\Gamma}}$-module isomorphism $\mathcal{O}_{T_{\Sigma}^{\Gamma}}(D) \cong \mathcal{O}_{T_{\Sigma}^{\Gamma}}\left(D_{h}\right)$ for some $h \in \operatorname{SF}(N, \Sigma, \Gamma)$. The following are equivalent for $h \in \mathrm{SF}(N, \Sigma, \Gamma)$.

i. $h \in M$

ii. $D_{h}$ is a principal Cartier divisor.

iii. $\mathcal{L}_{D_{h}}$ is a trivial line bundle.

iv. The sheaf $\mathcal{O}_{T_{\Sigma}^{\Gamma}}\left(D_{h}\right)$ is isomorphic to $\mathcal{O}_{T_{\Sigma}^{\Gamma}}$ as $\mathcal{O}_{T_{\Sigma}^{\Gamma}}-$ module.

Proof. See Proposition 2.4 of [26].

Proposition 8.5. Suppose that $|\Sigma|=N_{\mathbf{R}}$. Then we have canonical isomorphisms

$$
\operatorname{SF}(N, \Sigma, \Gamma) / M \rightarrow \operatorname{Pic}\left(T_{\Sigma}^{\Gamma}\right) \rightarrow \operatorname{CDiv}_{T^{M}}\left(T_{\Sigma}^{\Gamma}\right) /\left\{\operatorname{div}\left(t^{m}\right)\right\}_{m \in M},
$$

from which we deduce a canonical injection $\operatorname{Pic}\left(T_{\Sigma}^{\Gamma}\right) \rightarrow \operatorname{Pic}\left(T_{\Sigma}\right)$. 
Proof. This follows by using the same arguments as in Corollary 2.5 26].

If $\rho$ belongs to the 1 -skeleton $\Sigma(1)$ of the fan $\Sigma$ we denote by $\nu_{\rho}$ the primitive integral vector for the lattice $N$ in the ray $\rho$, that is the generator of the semigroup $\rho \cap N$. We associate to $h \in \mathrm{SF}(N, \Sigma)$ the polyhedron

$$
P_{h}:=\left\{m \in M_{\mathbf{R}} \mid\left\langle\nu_{\rho}, m\right\rangle \geq h\left(\nu_{\rho}\right), \rho \in \Sigma(1)\right\} .
$$

Recall that

$$
P_{l h}=l P_{h} \text { and } P_{h}+P_{h^{\prime}}=P_{h+h^{\prime}}
$$

for any integer $l \geq 1$ and $h, h^{\prime} \in \operatorname{SF}(N, \Sigma)$.

Proposition 8.6. Suppose that $|\Sigma|=N_{\mathbf{R}}$. The following are equivalent for $h \in$ $S F(N, \Sigma, \Gamma)$ defining a Cartier divisor $D=D_{h}$.

i. The $\mathcal{O}_{T_{\Sigma}^{\Gamma}}$-module $\mathcal{O}_{T_{\Sigma}^{\Gamma}}(D)$ is generated by its global sections.

ii. $h$ is upper convex, i.e., $h(\nu)+h\left(\nu^{\prime}\right) \leq h\left(\nu+\nu^{\prime}\right)$ for all $\nu, \nu^{\prime} \in N_{\mathbf{R}}$.

iii. The polytope $P_{h}$ has vertices $\left\{m_{\sigma} \mid \sigma \in \Sigma\right\}$.

If these conditions hold the convex hull of the set $P_{D}^{\Gamma}$ is the polytope $P_{h}$ and $h$ is the support function of the polytope $P_{h}$.

Proof. The proof follows as in the normal case (see Theorem 2.7 [26]).

If $|\Sigma|=N_{\mathbf{R}}$ the support function $h \in \mathrm{SF}(N, \Sigma, \Gamma)$, defined by the Cartier data $\left\{m_{\sigma} \mid \sigma \in \Sigma\right\}$, is strictly upper convex if it is upper convex and in addition

$$
h(\nu)=\left\langle\nu, m_{\sigma}\right\rangle \text { if and only if } \nu \in \sigma \text {, for } \sigma \in \Sigma .
$$

Suppose that $h \in \mathrm{SF}(N, \Sigma, \Gamma)$ satisfies the equivalent conditions of Proposition 8.6. Set $D=D_{h}$. If $P_{D}^{\Gamma}=\left\{u_{1}, \ldots, u_{s}\right\}$ we have a morphism

$$
\Phi_{D}: T_{\Sigma}^{\Gamma} \longrightarrow \mathbf{P}_{k}^{s-1}, \quad \Phi_{D}=\left(t^{u_{1}}: \cdots: t^{u_{s}}\right)
$$

(defined in homogeneous coordinates of $\mathbf{P}_{k}^{s-1}$ ). The morphism $\Phi_{D}$ is equivariant with respect the map of tori $\Phi_{\mid T^{M}}: T^{M} \rightarrow T^{M^{\prime}}$, where $T^{M^{\prime}}$ denotes the torus of $\mathbf{P}_{k}^{s-1}$ with respect to the fixed coordinates.

Proposition 8.7. Suppose that $|\Sigma|=N_{\mathbf{R}}$. The following are equivalent for $h \in$ $S F(N, \Sigma, \Gamma)$ defining a Cartier divisor $D=D_{h}$.

i. $D$ is very ample.

ii. $h$ is strictly upper convex and for all $\sigma \in \Sigma(d)$ the set $\left\{m-m_{\sigma} \mid m \in P_{D}^{\Gamma}\right\}$ generates the semigroup $\Gamma_{\sigma}$.

Proof. Suppose that $h$ is not strictly upper convex. Then there exists $d$-dimensional cones $\sigma, \sigma^{\prime} \in \Sigma$ such that $\tau=\sigma \cap \sigma^{\prime}$ is of dimension $d-1$ and $m_{\sigma}=m_{\sigma^{\prime}}$. This implies that the section defined by $t^{m_{\sigma}}$ in the open set $U=T^{\Gamma_{\sigma}} \cup T^{\Gamma_{\sigma^{\prime}}}$ is nowhere vanishing.

By definition there exists $1 \leq i \leq s$ such that $m_{\sigma}=u_{i}$.

The restriction of $\Phi_{D}$ to $U$ factors through the affine open set $\mathbf{C}^{s-1}$, where the $i$-th homogeneous coordinate does not vanish. It is of the form:

$$
\Phi_{\mid U}: U \rightarrow \mathbf{C}^{s-1}, \text { with } \Phi_{\mid U}=\left(t^{u_{1}-m_{\sigma}}, \ldots, t^{u_{i-1}-m_{\sigma}}, t^{u_{i+1}-m_{\sigma}}, \ldots, t^{u_{s}-m_{\sigma}}\right) .
$$

By Lemma 3.5 the closure of the orbit $\operatorname{orb}(\tau, \Gamma)$ is a complete one-dimensional toric variety contained in $U$. The restriction $\Phi_{\sqrt{\operatorname{orb}(\tau, \Gamma)}}$ must be constant hence $\Phi$ is not an embedding. This implies that if $D_{h}$ is very ample $h$ is strictly upper convex.

Suppose that $h$ is strictly upper convex. If $\sigma \in \Sigma$ is a $d$-dimensional cone then $m_{\sigma}$ belongs to $\left\{u_{i}\right\}_{i=1}^{s}$, say $m_{\sigma}=u_{s}$. The restriction of $\Phi$ to $T^{\Gamma_{\sigma}}$ factors though the affine 
open set of $\mathbf{P}_{k}^{s-1}$ where the last homogeneous coordinate does not vanish. It is described algebraically by the homomorphism of $k$-algebras:

$$
k\left[y_{1}, \ldots, y_{s-1}\right] \rightarrow k\left[t^{\Gamma}\right], \quad y_{i} \mapsto t^{u_{i}-m_{\sigma}}, i=1, \ldots, s-1 .
$$

This maps defines a closed immersion if and only if it is surjective. This happens if and only if the set of vectors $\left\{u_{i}-m_{\sigma}\right\}_{1 \leq i \leq s-1}$ generate the semigroup $\Gamma_{\sigma}$.

Proposition 8.8. Suppose that $|\Sigma|=N_{\mathbf{R}}$. The following are equivalent for $h \in$ $S F(N, \Sigma, \Gamma)$.

i. $D_{h}$ is ample

ii. $h$ is strictly upper convex.

Proof. If $D$ is ample then $l D$ is very ample for $l \gg 0$. Since $l D=D_{l h}$ it follows that $h$ is strictly upper convex if $l h$ is and the assertion holds by Proposition 8.7.

Conversely suppose that $h$ is strictly upper convex. We prove that $l D_{h}$ is very ample for $l \gg 0$. By Proposition 8.7 it is sufficient to prove that there exists an integer $l \gg 0$ such that for each $d$-dimensional cone $\sigma \in \Sigma$ the semigroup $\Gamma_{\sigma}$ is generated by $\left\{m-l m_{\sigma} \mid m \in P_{D_{l h}}^{\Gamma}\right\}$.

If $\sigma^{\prime} \in \Sigma, \operatorname{dim} \sigma^{\prime}=d, \tau=\sigma^{\prime} \cap \sigma$ we have that $\Gamma_{\tau}=\Gamma_{\sigma}+\mathbf{Z}_{\geq 0}(-u)$ for any $u \in \Gamma_{\sigma}$ in the relative interior of the cone $\tau^{\perp} \cap \check{\sigma}$ (see Lemma 3.9). For instance we take $u=m_{\sigma^{\prime}}-m_{\sigma}$. We obtain similarly that $\Gamma_{\tau}=\Gamma_{\sigma^{\prime}}+\mathbf{Z}_{\geq 0}(u)$.

If $\gamma \in \Gamma_{\sigma}$ then $\gamma$ belongs to $\Gamma_{\tau}$ and there exists $\gamma^{\prime} \in \Gamma_{\sigma^{\prime}}$ and an integer $p \geq 0$ such that $\gamma=\gamma^{\prime}+p u$. If $l \geq p$ we obtain:

$$
l m_{\sigma^{\prime}}+\gamma^{\prime}+(l-p)\left(m_{\sigma}-m_{\sigma^{\prime}}\right)=l m_{\sigma}+\gamma .
$$

If $l$ is big enough, a formula of the form (14) holds for any $\gamma$ in a finite set $G_{\sigma}$ of generators of $\Gamma_{\sigma}$ (where $p$ and $\gamma^{\prime}$ vary with $\gamma$ ) and for any cone $\sigma^{\prime} \in \Sigma(d)$. Since $\gamma^{\prime}$ and $m_{\sigma}-m_{\sigma^{\prime}}$ belong to $\Gamma_{\sigma^{\prime}}$ this implies $t^{l m_{\sigma}+\gamma}$ defines a section in $H^{0}\left(T^{\Gamma_{\sigma^{\prime}}}, \mathcal{O}_{T_{\Sigma}^{\Gamma}}\left(D_{l h}\right)\right)$ (see (8) ) for any cone $\sigma^{\prime} \in \Sigma(d)$. We deduce that for any $\gamma \in G_{\sigma}$ the vector $l m_{\sigma}+\gamma$ belongs to the set $P_{D_{l h}}^{\Gamma}$ and $t^{l m_{\sigma}+\gamma}$ defines a global section of $\mathcal{O}_{T_{\Sigma}^{\Gamma}}\left(D_{l h}\right)$.

Remark 8.9. Let $\mathcal{A}=\left\{u_{1}, \ldots, u_{l}\right\}$ be a subset of a lattice $M$ such that $\mathbf{Z} \mathcal{A}=M$, i.e., $\mathcal{A}$ spans $M$ as a lattice. Gel'fand, Kapranov, and Zelevinsky [12] define a projective toric variety $X_{\mathcal{A}}$ as the closure of the image of the map

$$
\varphi_{\mathcal{A}}=\left(t^{u_{1}}: \ldots: t^{u_{l}}\right): T^{M} \rightarrow \mathbf{P}_{k}^{l-1} .
$$

Let us explain how their definition fits with our notion of projective toric variety. Let $P$ be the convex hull of $\mathcal{A}$ in $M_{\mathbf{R}}$ and $\Sigma$ the dual fan of $P$. Each $\sigma \in \Sigma$ of maximal dimension determines a vertex $m_{\sigma}$ of $P$, which is necessarily an element of $\mathcal{A}$. We associate to $\sigma$ the semigroup $\Gamma_{\sigma}:=\sum \mathbf{Z}_{\geq 0}\left(u_{i}-m_{\sigma}\right)$. If $\tau \leq \sigma$ we define $\Gamma_{\tau}$ by (2). The set $\Gamma:=\left\{\Gamma_{\theta} \mid \theta \in \Sigma\right\}$ is well-defined and the triple $(N, \Sigma, \Gamma)$ defines a toric variety $T_{\Sigma}^{\Gamma}$ (the argument is the same as the one used in the proof of Proposition 5.1). The support function $h$ of $P$ belongs to $\operatorname{SF}(N, \Sigma, \Gamma)$ and is strictly upper convex. If $D=D_{h}$ we deduce from the definitions that $\mathcal{A} \subset P_{D}^{\Gamma}$. By Proposition 8.7 the Cartier divisor $D$ is very ample, and the morphism (13) is an equivariant embedding of $T_{\Sigma}^{\Gamma}$ in the projective space. It follows that $X_{\mathcal{A}}$ and $T_{\Sigma}^{\Gamma}$ are isomorphic toric varieties. Notice that the embedding defined by (13) may be degenerate, that is, the image may lie in a proper linear subspace.

Remark 8.10. If $F=\sum_{i=1}^{s} c_{i} t^{u_{i}} \in k\left[t^{M}\right]$ is a polynomial with $c_{1} \ldots c_{s} \neq 0$, then $F$ defines a global section of $\mathcal{O}_{T_{\Sigma}^{\Gamma}}(D)$ such that the closure of $\{F=0\} \cap T^{M}$ in $T_{\Sigma}^{\Gamma}$ does not meet any zero-dimensional orbit of $T_{\Sigma}^{\Gamma}$. 


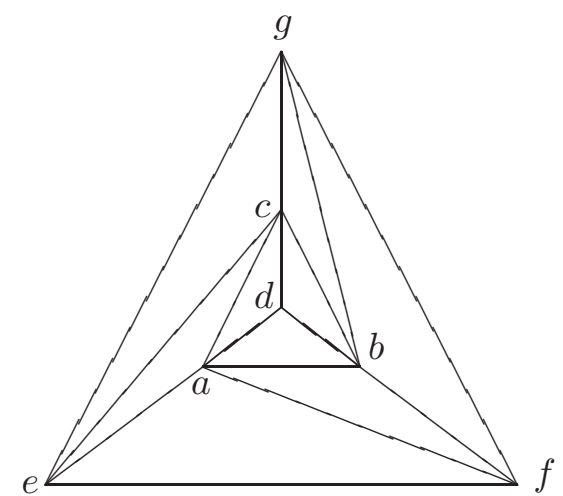

FiguRE 1. The cones of $\Sigma$ subdividing the first orthant in Example 8.12

Proposition 8.11. Suppose that $|\Sigma|=N_{\mathbf{R}}$. Then the toric variety $T_{\Sigma}^{\Gamma}$ is projective if and only if its normalization $T_{\Sigma}$ is projective.

Proof. Suppose that $T_{\Sigma}$ is projective. Then there exists a strictly upper convex function $h \in S F(N, \Sigma)$. By definition, there exists an integer $k_{0} \geq 1$ such that $k_{0} h \in$ $S F(N, \Sigma, \Gamma)$. It follows that $T_{\Sigma}^{\Gamma}$ is projective by Proposition 8.8 ,

If $T_{\Sigma}^{\Gamma}$ is projective there exists a strictly upper convex function $h \in S F(N, \Sigma, \Gamma)$. Since $S F(N, \Sigma, \Gamma) \subset S F(N, \Sigma)$ it follows that $T_{\Sigma}$ is projective.

Example 8.12. We give an example of complete non-normal toric variety $T_{\Sigma}^{\Gamma}$, which is non projective. We recall first the Example 6.1.17 of [5] of a complete smooth toric variety $T_{\Sigma}$, which is non-projective. The maximal faces of the fan $\Xi$ associated to $\left(\mathbf{P}^{1}\right)^{3}$ are the eight orthants of $\mathbf{R}^{3}$. We denote the canonical basis of $\mathbf{Z}^{3}$ by $e, f, g$ and we set $N=\mathbf{Z}^{3}$. In term of this basis we consider the vectors $a=(2,1,1), b=(1,2,1)$, $c=(1,1,2)$ and $d=(1,1,1)$. We define from $\Xi$ a complete fan $\Sigma$, by subdividing the first orthant $\mathbf{R}_{\geq 0}^{3}=\mathbf{R}_{\geq 0} e+\mathbf{R}_{\geq 0} f+\mathbf{R}_{\geq 0} g$, adding the rays defined by $a, b, c, d$ in the way indicated in the Figure 1 .

Let us denote by $a^{*}, c^{*}, d^{*}$ (resp. $\left.a^{\prime}, c^{\prime}, e^{\prime}\right)$ the dual basis of $a, c, d$ (resp. of $\left.a, c, e\right)$. In term of these basis of $M$ we introduce the following non-saturated semigroups:

$$
\begin{aligned}
& \Gamma_{a c d}:=a^{*} \mathbf{Z}_{\geq 0}+c^{*} \mathbf{Z}_{\geq 0}+\left(a^{*}+d^{*}\right) \mathbf{Z}_{\geq 0}+\left(c^{*}+d^{*}\right) \mathbf{Z}_{\geq 0}+2 d^{*} \mathbf{Z}_{\geq 0}, \\
& \Gamma_{a c}:=a^{*} \mathbf{Z}_{\geq 0}+c^{*} \mathbf{Z}_{\geq 0}+\left(a^{*}+d^{*}\right) \mathbf{Z}_{\geq 0}+\left(c^{*}+d^{*}\right) \mathbf{Z}_{\geq 0}+2 d^{*} \mathbf{Z}, \\
& \Gamma_{a c e}:=a^{\prime} \mathbf{Z}_{\geq 0}+c^{\prime} \mathbf{Z}_{\geq 0}+\left(a^{\prime}+e^{\prime}\right) \mathbf{Z}_{\geq 0}+\left(c^{\prime}+e^{\prime}\right) \mathbf{Z}_{\geq 0}+2 e^{\prime} \mathbf{Z}_{\geq 0}
\end{aligned}
$$

Let us denote by $\sigma_{a c d}$ the cone $\mathbf{R}_{\geq 0} a+\mathbf{R}_{\geq 0} c+\mathbf{R}_{\geq 0} d$. We use a similar notation to define the cones $\sigma_{a c e}$ and $\sigma_{a c}$. Let us define a semigroup $\Gamma_{\sigma}$ associated to each cone $\sigma \in \Sigma$ by:

$$
\Gamma_{\sigma}:=\left\{\begin{array}{lllll}
\Gamma_{a c d} & \text { if } & \sigma & = & \sigma_{a c d} \\
\Gamma_{a c e} & \text { if } & \sigma & = & \sigma_{a c e} \\
\Gamma_{a c} & \text { if } & \sigma & = & \sigma_{a c} \\
\check{\sigma} \cap M & & & \text { otherwise. }
\end{array}\right.
$$

Then the triple $(N, \Sigma, \Gamma)$ satisfies the conditions in the definition 4.1; It is immediate that $\mathbf{Z} \Gamma_{\sigma}=M$ and $\mathbf{R}_{\geq 0} \Gamma_{\sigma}=\check{\sigma}$, for any $\sigma \in \Sigma$. We check the compatibility conditions among those semigroups defining different charts. First, if $\tau \neq \sigma_{a c}$ is a proper face of $\sigma_{a c d}$ then we get that $\Gamma_{a c d}+M\left(\tau, \Gamma_{a c d}\right)=\check{\tau} \cap M$ is a regular semigroup. The same assertion holds replacing acd by ace. In these cases the compatibility conditions are the same as in the normal case. It remains to check what happens when $\tau=\sigma_{a c}$ is the common face of $\sigma_{a c d}$ and $\sigma_{a c e}$. One gets that $\Gamma_{a c d}+M\left(\tau, \Gamma_{a c d}\right)=\Gamma_{a c}$ while $\Gamma_{a c e}+M\left(\tau, \Gamma_{a c e}\right)$ is the semigroup $\Gamma_{a c}^{\prime}$ generated by $a^{\prime}, c^{\prime}, a^{\prime}+e^{\prime}, c^{\prime}+e^{\prime}, \pm 2 e^{\prime}$. Since 
$c^{\prime}=c^{*}, a^{\prime}=a^{*}+d^{*}$ and $e^{\prime}=-d^{*}$ it follows that $\Gamma_{a c}=\Gamma_{a c}^{\prime}$, hence all the compatibility conditions hold. By Proposition 8.11 we deduce that the complete toric variety $T_{\Sigma}^{\Gamma}$ is non-normal and non-projective.

\section{Part II: Local uniformization of maximal rank monomial valuations on toric varieties by Semple-Nash modifications}

In this Part we prove that a maximal rank monomial valuation dominating a point of a toric variety admits a canonical local uniformization by a finite number of iterated blowing ups of logarithmic jacobian ideals. Recall that, as shown below, if $k$ is an algebraically closed field of characteristic zero the blowing up of the logarithmic jacobian ideal of an affine toric variety $T^{\Gamma}$ coincides with the Semple-Nash modification. This fact, originally due to Gérard Gonzalez Sprinberg in the normal case ([14]), is our starting point.

The sequence of logarithmic jacobian blowing-ups of a toric variety $T_{\Sigma^{(0)}}^{\Gamma^{(1)}}$ is a sequence of toric varieties $T_{\Sigma^{(i)}}^{\Gamma^{(i+1)}}$ defined by a sequence $\Sigma^{(i)}$ of refinements (or subdivisions) of $\Sigma$ with attached families $\Gamma^{(i+1)}$ of semigroups. The center of a monomial valuation of maximal rank $d$ on the toric variety $T_{\Sigma^{(i)}}^{\Gamma^{(i+1)}}$ is a zero dimensional orbit corresponding to a $d$-dimensional cone $\tau^{(i)}$ of the fan $\Sigma^{(i)}$. The main result of this part shows that the affine toric variety $T_{\tau^{(i)}}^{\Gamma^{(i+1)}}$ becomes smooth after finitely many iterations of logarithmic jacobian blowing-ups (see Theorem 12.35).

In most proofs of resolution the strategy is to attach to points an invariant which takes its minimal value only for regular points and then show that it can be made to decrease by successive blowing-ups. Our strategy is different: we show that the very nature of the blowing-up of the logarithmic jacobian ideals forces the cones distinguished by the valuation in the successive refinements $\Sigma^{(i)}$ of $\Sigma$ to stabilize for $i$ large enough, meaning that they are not subdivided in the $\Sigma^{(j)}$ for $j \geq i$. If one can stabilize the cones of maximal dimension the logarithmic jacobian blowing-ups are finite morphisms from then on, and it is easy to show that they resolve in finitely many steps (see Proposition 12.20). This stabilization is not measured by the constancy of some local invariant. The basic idea is to show stabilization by extending it from lower-dimensional cones to higher-dimensional ones, so that if one really insists on having an invariant, it should be the minimal codimension of stable faces of the cone $\tau^{(i)}$ picked by the valuation; it is at most $d-1$ since edges are stable, and if it is zero, we are essentially done.

Here is a quick description of the structure of the proof: first we study the problem with respect to the monomial valuation associated to a vector $\nu \in \sigma \cap N$, where $\sigma \in \Sigma(d)$. For each $j$ such a vector determines a unique cone $\theta^{(j)} \in \Sigma^{(j)}$ containing $\nu$ in its relative interior, and the first observation is that this sequence $\left(\theta^{(j)}\right)_{j \geq 0}$ stabilizes for $j \geq j_{1}$ say; the limit $\theta^{(\infty)}=\theta^{\left(j_{1}\right)}$ is by definition a stable cone of $\Sigma^{\left(j_{1}\right)}$. This implies that the chart $T_{\theta^{(\infty)}}^{\Gamma^{(j)}}$ is non-singular for $j \gg 0$ (see Propositions 12.20 and 12.22).

One of the difficulties is that the logarithmic jacobian blowing-up of the variety does not induce the logarithmic jacobian blowing-up of its lower-dimensional orbit closures. A key point in the proof is that given a stable cone $\eta$, a nested sequence of cones $\zeta^{(j)} \in \Sigma^{(j)}$ containing $\eta$ as a codimension one face necessarily stabilizes (Proposition 12.25). This uses the fact that for every one dimensional orbit closure associated to a stable cone of codimension one, the effect of the ambient blowing-up is very similar that of its logarithmic jacobian blowing-up (see Claim 12.26). A monomial valuation of maximal rank defines a nested sequence of $d$-dimensional cones $\tau^{(j)} \in \Sigma^{(j)}$. We show first that this sequence contains a stable cone $0 \neq \eta \leq \tau^{(j)}$ for $j \gg 0$. If $\eta \neq \tau^{(j)}$ 
we analyze the the blowing up of the logarithmic jacobian ideal on the orbit closure associated to $\eta$ on the chart picked up by the valuation and we prove that after finitely many iterations we obtain a stable cone $\eta \leq \theta \leq \tau^{(j)}$ of smaller codimension.

In Sections 13 and 14 we give an interpretation of the main result in terms of the Zariski-Riemann space of a fan, introduced by Ewald and Ishida (10]).

The recent paper [1] suggests that it would be interesting to develop an approach from a computational viewpoint to the iteration of Semple-Nash modification.

\section{The Semple-Nash modification: preliminaries}

In [27, Semple introduced the Semple-Nash modification of an algebraic variety and asked whether a finite number of iterations would resolve the singularities of the variety. The same question was apparently rediscovered by Chevalley and Nash in the 1960's, and studied notably by Nobile (see 24]), Gonzalez-Sprinberg (see 16 and 14]), Hironaka (see 19]), and Spivakovsky (see 29]). The best consequence so far of all this work is the Theorem, due to Spivakovsky, stating that by iterating the operation consisting of the Semple-Nash modification followed by normalization one eventually resolves singularities of surfaces over an algebraically closed field of characteristic zero.

Let $X$ be a reduced algebraic variety or analytic space, which we may assume of pure dimension $d$ for simplicity. Whenever we speak of the Semple-Nash modification, we assume that we are working over an algebraically closed field $k$ of characteristic zero. Consider the Grassmanian $g: \operatorname{Grass}_{d} \Omega_{X}^{1} \rightarrow X$; it is a proper algebraic map, which has the property that its fiber over a point of $x$ is the Grassmanian of $d$-dimensional subspaces of the Zariski tangent space $E_{X, x}$. The map $g$ is characterized by the fact that $g^{*} \Omega_{X}^{1}$ has a locally free quotient of rank $d$ and $g$ factorizes in a unique manner every map to $X$ with this property. Let $X^{o}$ denote the non-singular part of $X$, which is $d$ dimensional and dense in $X$ by our assumptions. Since the restriction $\Omega_{X}^{1} \mid X^{o}$ is locally free the map $g$ has an algebraic section over $X^{o}$ and the Semple-Nash modification is defined as the closure $N X$ of the image of this section, endowed with the natural projection $n_{X}: N X \rightarrow X$ induced by $g$. The map $n_{X}$ is proper and is an isomorphism over $X^{o}$; it is a modification. Like the Grassmanian of $\Omega_{X}^{1}$, it is defined up to a unique $X$-isomorphism.

A local description can be given for a chart $X \mid U$ of $X$ embedded in affine space $\mathbf{A}^{N}(k)$ by taking the closure in $(X \mid U) \times \mathbf{G}(N, d)$ of the graph of the Gauss map $\gamma:(X \mid U)^{o} \rightarrow \mathbf{G}(N, d)$ sending each non-singular point to the class of its tangent space in the Grassmanian of $d$-dimensional vector subspaces in $\mathbf{A}^{N}(k)$. For any point $x \in X$ the fiber $n_{X}^{-1}(x)$ is the subset of $\mathbf{G}(N, d)$ consisting of limit positions at $x \in X$ of tangent spaces to $X$ along sequences of non-singular points tending to $x$. In this guise, the Semple-Nash modification appears in a complex-analytic framework in the paper 39. of Hassler Whitney in connection with equisingularity problems.

Proposition 9.1. (Nobile), see 24] and 33. Let $X$ be a reduced equidimensional space; if the map

$$
n_{X}: N X \rightarrow X
$$

is an isomorphism, the space $X$ is non-singular.

For the convenience of the reader, we sketch the proof found in 33:

If the map $n_{X}$ is an isomorphism, the sheaf $\Omega_{X}^{1}$ has a locally free quotient of rank $d$. The problem is local, so it is enough to prove that the existence of a surjective map $\phi: \Omega_{X, x}^{1} \rightarrow \mathcal{O}_{X, x}^{d}$ implies, in characteristic zero, that $\mathcal{O}_{X, x}$ is regular. Passing to the completion and tensoring $\Omega_{X, x}^{1}$ by $\hat{\mathcal{O}}_{X, x}$ we may assume that $\mathcal{O}_{X, x}$ is complete. We consider the linear map $e: \mathcal{O}_{X, x}^{d} \rightarrow \mathcal{O}_{X, x}$ sending the first basis vector to 1 and the 
others to 0 . The composition of $e$ with the map $\phi$ gives a surjective map, so that there has to be an element $h \in \mathcal{O}_{X, x}$ such that the image of $d h$ in $\mathcal{O}_{X, x}$ by $e \circ \phi$ is equal to 1 , and then the $k$-derivation $D: \mathcal{O}_{X, x} \rightarrow \mathcal{O}_{X, x}$ corresponding to $e \circ \phi$ is such that $D h=1$. In characteristic zero one can formally integrate this non vanishing vector field using the formal expansion of $\exp (-h D)$ to get an isomorphism $\mathcal{O}_{X, x} \simeq \mathcal{O}_{1}[[h]]$ where $\mathcal{O}_{1} \simeq \mathcal{O}_{X, x} /(h)$. By construction $\mathcal{O}_{1}$ satisfies the same assumptions as $\mathcal{O}_{X, x}$ in one less dimension. By induction we are reduced to dimension zero, but a reduced zero dimensional complete equicharacteristic local ring is $k$ in our case. We refer to 33 for details, and to 24] for the original proof.

Remark 9.2. We will see below in Section 11, Proposition 11.3, the characteristic-free version of this statement, which is that if the blowing-up of the logarithmic jacobian ideal is an isomorphism, the toric variety is smooth. Note that the Semple-Nash modification is defined in any characteristic but its being an isomorphism does not imply regularity in positive characteristic; it is the case for $y^{p}-x^{q}=0$ with $(p, q)=1$ in characteristic $p$. See [24].

\section{The Semple-Nash modification in the toric CASE}

The following is an extension to the case of not necessarily normal toric varieties of a result of Gonzalez-Sprinberg ([14]; a summary of this work appeared in [15]) which was revisited by Lejeune-Jalabert and Reguera in the appendix to 23 .

Let $X$ be an affine toric variety over an algebraically closed field $k$. Using the notations of Section 3 we write its ring

$$
R=k\left[U_{1}, \ldots, U_{r}\right] / P,
$$

where $P$ is a prime binomial ideal $\left(U^{m^{\ell}}-U^{n^{\ell}}\right)_{\ell \in \mathbf{L}}$ of the polynomial ring $k\left[U_{1}, \ldots, U_{r}\right]$. Let $d$ be the dimension of $X$ and denote by $\mathcal{L} \subset \mathbf{Z}^{r}$ the lattice generated by the differences $\left(m^{\ell}-n^{\ell}\right)_{\ell \in \mathbf{L}}$; by [8], it is a direct factor of $\mathbf{Z}^{r}$ since $X$ is irreducible and $k$ is algebraically closed. Setting $c=r-d$, we may identify $\mathbf{L}$ with $\{1, \ldots, L\}$ with $L=|\mathbf{L}|$ in such a way that the lattice generated by $\left(m^{1}-n^{1}, \ldots, m^{c}-n^{c}\right)$ has rank $c$. The quotient $\mathbf{Z}^{r} / \mathcal{L}$ is isomorphic to $\mathbf{Z}^{d}$ and we have an exact sequence

$$
0 \rightarrow \mathcal{L} \stackrel{\psi}{\rightarrow} \mathbf{Z}^{r} \rightarrow \mathbf{Z}^{d} \rightarrow 0 .
$$

Our affine toric variety $X$ is Speck $\left[t^{\Gamma}\right]$, where $\Gamma$ is the semigroup generated in $\mathbf{Z}^{d}$ by the images $\gamma_{1}, \ldots, \gamma_{r}$ of the basis vectors of $\mathbf{Z}^{r}$. The logarithmic jacobian ideal of $X$ is the ideal of $R=k\left[t^{\Gamma}\right]$ generated by the images of the products $U_{i_{1}} \ldots U_{i_{d}}$ such that $\operatorname{Det}\left(\gamma_{i_{1}}, \ldots, \gamma_{i_{d}}\right) \neq 0$.

Proposition 10.1. (Generalizing [14] [15] and [23]) Let $X$ be an affine toric variety over an algebraically closed field of characteristic zero. The Semple-Nash modification of $X$ is isomorphic to the blowing-up its logarithmic jacobian ideal.

Proof. Keeping the notations just introduced, a straightforward computation using logarithmic differentials shows that the jacobian determinant $J_{K, \mathbf{L}^{\prime}}$ of rank $c=r-d$ of the generators $\left(U^{m^{\ell}}-U^{n^{\ell}}\right)_{\ell \in\{1, \ldots, L\}}$ of our prime binomial ideal $P \subset k\left[U_{1}, \ldots, U_{r}\right]$, associated to a sequence $K=\left(k_{1}, \ldots, k_{c}\right)$ of distinct elements of $\{1, \ldots, r\}$ and a subset $\mathbf{L}^{\prime} \subseteq\{1, \ldots, L\}$ of cardinality $c$, satisfies the congruence

$$
U_{k_{1}} \ldots U_{k_{c}} \cdot J_{K, \mathbf{L}^{\prime}} \equiv\left(\prod_{\ell \in \mathbf{L}^{\prime}} U^{m^{\ell}}\right) \operatorname{Det}_{K, \mathbf{L}^{\prime}}((\langle m-n\rangle)) \bmod . P,
$$


where $(\langle m-n\rangle)$ is the matrix of the vectors $\left(m^{\ell}-n^{\ell}\right)_{\ell \in\{1, \ldots, L\}}$, and $\operatorname{Det}_{K, \mathbf{L}^{\prime}}$ indicates the minor in question. By Lemma 6.3 of [34], the rank of the image in $k^{r \times L}$ of the matrix $(\langle m-n\rangle)$ is equal to $c$.

By (24, proof of Th.1) the Nash modification of $X$ is isomorphic to the blowing up in $X$ of the ideal generated as $K=\left(k_{1}, \ldots, k_{c}\right)$ runs through the sets of $c$ distinct elements of $(1, \ldots, r)$ by the elements $J_{K, \mathbf{L}_{0}}$ satisfying the congruences

$$
U_{k_{1}} \ldots U_{k_{c}} \cdot J_{K, \mathbf{L}_{0}} \equiv\left(\prod_{\ell \in \mathbf{L}_{0}} U^{m^{\ell}}\right) \operatorname{Det}_{K, \mathbf{L}_{0}}((\langle m-n\rangle)) \bmod . P
$$

where $\mathbf{L}_{0}=(1, \ldots, c)$ is, after renumbering of $\{1, \ldots, L\}$, a subset such that these jacobian determinants are not all zero; such subsets exist since the $J_{K, \mathbf{L}^{\prime}}$ are not all zero. Remark the necessity that $J_{K, \mathbf{L}_{0}}=0$ whenever the determinant on the right side is zero.

Remark also that by [3], we may not suppose that the first $c$ binomials define a complete intersection.

Now for each $K$ let us multiply both sides by $U_{i_{1}} \ldots U_{i_{d}}$, where $I=\left(i_{1}, \ldots, i_{d}\right)=$ $\{1, \ldots, r\} \backslash K$. We obtain for each $K$ the equality:

$$
U_{1} \ldots U_{r} . J_{K, \mathbf{L}_{0}} \equiv U_{i_{1}} \ldots U_{i_{d}}\left(\prod_{\ell \in \mathbf{L}_{0}} U^{m^{\ell}}\right) \operatorname{Det}_{K, \mathbf{L}_{0}}((\langle m-n\rangle)) \bmod . P .
$$

Taking exterior powers for the map $\psi$ in the sequence (15) gives an injection

$$
0 \rightarrow \stackrel{r-d}{\Lambda} \stackrel{r-d}{\Lambda} \stackrel{r-d}{\longrightarrow} \stackrel{r-d}{\Lambda} \mathbf{Z}^{r}
$$

whose image is a primitive vector in ${ }^{r-d} \mathbf{Z}^{r}$ since it is a direct factor.

Let $\mathcal{L}_{0} \subset \mathcal{L}$ be the lattice generated by the differences $\left(m^{1}-n^{1}, \ldots, m^{c}-n^{c}\right)$, that is, corresponding to the first $c$ binomial equations. The image of its $(r-d)$-th exterior power is a non-zero multiple of the primitive vector ${ }^{r-d} \Lambda$; all the $c \times c$ minors of the matrix $(\langle m-n\rangle)$ involving vectors $m^{\ell}-n^{\ell}$ with $\ell>c$ are rationally dependent upon those which do not. Consider now the $d$-th exterior power of the map dual to the surjection $\mathbf{Z}^{r} \rightarrow \mathbf{Z}^{d} \rightarrow 0$ of (15):

$$
0 \rightarrow \stackrel{d}{\Lambda} \check{\mathbf{Z}}^{d} \rightarrow \stackrel{d}{\Lambda} \check{\mathbf{Z}}^{r}
$$

The image of $\stackrel{d}{\Lambda} \check{\mathbf{Z}}$ is a primitive vector in $\stackrel{d}{\Lambda} \check{\mathbf{Z}}^{r}$.

By the natural duality isomorphism between $\stackrel{d}{\Lambda} \check{\mathbf{Z}}^{r}$ and $\stackrel{r-d}{\Lambda} \mathbf{Z}^{r}$ (see [2] $\S 11$, No. 11, Prop. 12) deduced from the pairings

$$
\stackrel{d}{\Lambda} \check{\mathbf{Z}}^{r} \otimes \stackrel{d}{\Lambda} \mathbf{Z}^{r} \rightarrow \mathbf{Z}, \stackrel{d}{\Lambda} \mathbf{Z}^{r} \otimes \stackrel{r-d}{\Lambda} \mathbf{Z}^{r} \rightarrow \mathbf{Z},
$$

this vector correspond to the image of $\Lambda^{r-d} \mathcal{L}$ in such a way that the coordinate which corresponds to the determinant of the vectors $\gamma_{i_{1}}, \ldots, \gamma_{i_{d}}$ in $\mathbf{Z}^{d}$ is a rational multiple of the determinant $\operatorname{Det}_{K, \mathbf{L}_{0}}((\langle m-n\rangle))$, which is non-zero since our base field is of characteristic zero.

Equation 16 now shows that the ideal of $R$ generated by the $J_{K, \mathbf{L}_{0}}$ differs from the ideal generated by the images of the products $U_{i_{1}} \ldots U_{i_{d}}$ such that $\operatorname{Det}\left(\gamma_{i_{1}}, \ldots, \gamma_{i_{d}}\right) \neq 0$ only by the product by invertible ideals, so that these two ideals determine isomorphic blowing ups, which proves the Proposition. 
Remark 10.2. The proof found in 23 is valid in the non-normal case; the proof given here makes explicit the connection of the logarithmic jacobian ideal with the usual one.

Remark 10.3. The isomorphism of Proposition 1.8 carries the logarithmic jacobian ideal of $k\left[t^{\Gamma \times \Gamma^{\prime}}\right]$ onto the tensor product of the logarithmic jacobian ideals of the factors.

Remark 10.4. In the one-dimensional case the logarithmic jacobian ideal is the maximal ideal corresponding to the closed orbit. It is a classical fact that iterating the blowing-up of the singular point resolves the singularities of any branch.

\section{The SHEAF OF LOGARITHMIC JACOBIAN IDEALS ON A TORIC VARIETY}

Let the pair $(\Sigma, \Gamma)$ define a toric variety $T_{\Sigma}^{\Gamma}$ as in Definition 4.1 .

On the affine open set $T^{\Gamma_{\sigma}}, \sigma \in \Sigma$ we consider the ideal $\mathcal{J}_{\sigma}$ of $k\left[t^{\Gamma_{\sigma}}\right]$ generated by monomials of the form $t^{\alpha}$, where $\alpha$ belongs to the set

$$
\left|\mathcal{J}_{\sigma}\right|=\left\{\alpha_{1}+\cdots+\alpha_{d} \mid \alpha_{1}, \ldots, \alpha_{d} \in \Gamma_{\sigma} \text { and } \alpha_{1} \wedge \cdots \wedge \alpha_{d} \neq 0\right\} .
$$

As we saw in Proposition 10.1 the ideal $\mathcal{J}_{\sigma}$ is called the logarithmic jacobian ideal of $T^{\Gamma_{\sigma}}$.

Remark 11.1. If $\gamma_{1}, \ldots, \gamma_{r}$ are generators of the semigroup $\Gamma_{\sigma}$ then the monomials $t^{\alpha}$, for $\alpha$ in

$$
\left\{\gamma_{i_{1}}+\cdots+\gamma_{i_{d}} \mid \gamma_{i_{1}} \wedge \cdots \wedge \gamma_{i_{d}} \neq 0,1 \leq i_{1}, \ldots, i_{d} \leq r\right\},
$$

generate the ideal $\mathcal{J}_{\sigma}$. Abusing notation we denote the set (17) with the same letter $\mathcal{J}_{\sigma}$, whenever the set of generators of $\Gamma_{\sigma}$ is clear from the context.

Proposition 11.2. The family $\left\{\mathcal{J}_{\sigma} \mid \sigma \in \Sigma\right\}$ defines a $T^{M}$-invariant sheaf of ideals $\mathcal{J}$ on $T_{\Sigma}^{\Gamma}$, which is called the sheaf of logarithmic jacobian ideals of $T_{\Sigma}^{\Gamma}$.

Proof. It is sufficient to check that if $\tau \leq \sigma, \sigma \in \Sigma$, then the ideal $\mathcal{J}_{\tau}$ coincides with the extension $\mathcal{J}_{\sigma} k\left[t^{\Gamma_{\tau}}\right]$, induced by the inclusion $k\left[t^{\Gamma_{\sigma}}\right] \hookrightarrow k\left[t^{\Gamma_{\tau}}\right]$ defined by $\Gamma_{\sigma} \subset \Gamma_{\tau}$.

By Lemma 3.9 if $m \in \Gamma_{\sigma}$ belongs to the relative interior of the cone $\check{\sigma} \cap \tau^{\perp}$ then we have that $\Gamma_{\tau}=\Gamma_{\sigma}+\mathbf{Z}_{\geq 0}(-m)$.

If $\gamma_{1}, \ldots, \gamma_{r}$ are generators of $\Gamma_{\sigma}$ then $\gamma_{1}, \ldots, \gamma_{r},-m$ are generators of $\Gamma_{\tau}$. This implies the inclusion $\mathcal{J}_{\sigma} \subset \mathcal{J}_{\tau}$. By Remark 11.1 an exponent $\alpha$ in $\mathcal{J}_{\tau}$ which does not belong to the set $\mathcal{J}_{\sigma}$ is of the form: $\alpha=\gamma_{i_{1}}+\cdots+\gamma_{i_{d-1}}-m$, with $\gamma_{i_{1}} \wedge \cdots \wedge \gamma_{i_{d-1}} \wedge(-m) \neq$ 0 . Then, the element $\beta:=\gamma_{i_{1}}+\cdots+\gamma_{i_{d-1}}+m$ belongs to $\mathcal{J}_{\sigma}$ and we obtain that: $t^{\alpha}=t^{-2 m} t^{\beta} \in \mathcal{J}_{\sigma} k\left[t^{\Gamma_{\tau}}\right]$, and $\mathcal{J}_{\sigma} k\left[t^{\Gamma_{\tau}}\right]=\mathcal{J}_{\tau}$.

Proposition 11.3. The toric variety $T_{\Sigma}^{\Gamma}$ is non-singular if and only if the blowing up of the logarithmic jacobian ideal is an isomorphism.

Proof. We only have to prove that if the blowing up of the logarithmic jacobian ideal of an affine toric variety is an isomorphism the variety is smooth. We deal first with the case of a semigroup $\Gamma$ such that the cone $\check{\sigma}$ generated by $\Gamma$ is strictly convex, or equivalently that the cone $\sigma$ is of dimension $d=\operatorname{rank} M \geq 1$. In this situation the semigroup $\Gamma$ has a unique minimal system of generators $\gamma_{1}, \ldots, \gamma_{d}, \gamma_{d+1}, \ldots$ (see [9], Chapter V, Lemma 3.5, page 155. The result is proved there for $\check{\sigma} \cap M$ but the same argument applies to $\Gamma$ ). If there are more than $d$ generators, we may assume that the first $d$ generators are linearly independent. Then $\gamma_{d+1}$ is linearly dependent on the previous ones which gives us another element $m=\gamma_{1}+\cdots+\gamma_{i-1}+\gamma_{d+1}+\gamma_{i+1}+\cdots+\gamma_{d}$ of our ideal. Our assumption ensures that $m-m^{(1)}=\gamma_{d+1}-\gamma_{i}$ (or its opposite) is in $\Gamma$, which contradicts the assumption of minimality since the appearance of $\gamma_{d+1}$ in the right hand side of an expression $\gamma_{d+1}-\gamma_{i}=\sum a_{k} \gamma_{k}, a_{k} \in \mathbf{N}$, would contradict the strict convexity of the cone $\check{\sigma}$ by implying either that some positive multiple of $-\gamma_{d+1}$ 
is in $\Gamma$ or that $-\gamma_{i}$ is in $\Gamma$. The same argument works for $\gamma_{i}-\gamma_{d+1}$. Therefore $\Gamma$ has $d$ independent generators which generate $M$ and $k\left[t^{\Gamma}\right]$ is a polynomial ring.

If the dimension of $\sigma$ is $<d$ we deduce from the assumption and Proposition 12.22. ii) below that $M(\sigma, \Gamma)=M(\sigma)$. Then we reduce to the case $\operatorname{dim} \sigma=d$ by Lemma 12.18 below. Neither of those two results uses this proposition.

Lemma 11.4. There is a continuous piecewise linear function $\operatorname{ord}_{\mathcal{J}}:|\Sigma| \rightarrow \mathbf{R}$ such that for each $\tau \in \Sigma$ the function $\operatorname{ord}_{\mathcal{J}_{\tau}}$ is the restriction of $\operatorname{ord}_{\mathcal{J}}$ to $\tau$.

Proof. This follows from the definition of $\operatorname{ord}_{\mathcal{J}_{\sigma}}$ (see (3) ), by using that $\mathcal{J}$ is a sheaf of monomial ideals.

Remark 11.5. Note that Lemma 11.4 holds more generally if we replace $\mathcal{J}$ by any sheaf of monomial ideals $\mathcal{I}$ on $T_{\Sigma}^{\Gamma}$.

The will need the following lemma in Section 12

Lemma 11.6. Let $\theta^{\perp} \cap \Gamma$ be a face of the finitely generated semigroup $\Gamma \subset M$. The logarithmic jacobian ideal $\tilde{\mathcal{J}}$ of the image $\tilde{\Gamma}$ of $\Gamma$ in the lattice $M / M(\theta)$ is equal to the image of the logarithmic jacobian ideal $\mathcal{J}$ of $\Gamma$.

Proof Let us denote by $\tilde{\gamma}_{i}$ the images in $M / M(\theta)$ of the generators $\gamma_{i}$ of $\Gamma$ and by $p$ the rank of the lattice $M / M(\theta)$. If $\tilde{\gamma}_{i_{1}}, \ldots, \tilde{\gamma}_{i_{p}}$ are linearly independent in $M / M(\theta)$, then $\gamma_{i_{1}}, \ldots, \gamma_{i_{p}}$ must be linearly independent from $\theta^{\perp}$. Remark that since $\theta^{\perp} \cap \Gamma$ is a face it must contain $d-p$ generators of $\Gamma$ which are linearly independent, since $\theta^{\perp} \cap \Gamma$ spans the rank $d-p$ lattice $M(\theta, \Gamma)$. Choosing linearly independent generators $\gamma_{i_{p+1}}, \ldots, \gamma_{i_{d}} \in \theta^{\perp} \cap \Gamma$ of $\Gamma$ gives us a generator $\gamma_{i_{1}}+\cdots+\gamma_{i_{p}}+\gamma_{i_{p+1}}+\cdots+\gamma_{i_{d}}$ of $\mathcal{J}$ whose image is $\tilde{\gamma}_{i_{1}}+\cdots+\tilde{\gamma}_{i_{p}}$, showing that $\tilde{\mathcal{J}}$ is contained in the image of $\mathcal{J}$. If we now take $d$ independent generators $\gamma_{i_{1}}, \ldots, \gamma_{i_{p}}, \gamma_{i_{p+1}}, \ldots, \gamma_{i_{d}}$ of $\Gamma$, since they generate $M$, there must exist $d-p$ independent elements in their images, say $\tilde{\gamma}_{i_{1}}, \ldots, \tilde{\gamma}_{i_{p}}$. Then the image $\tilde{\gamma}_{i_{1}}+\cdots+\tilde{\gamma}_{i_{p}}+\tilde{\gamma}_{i_{p+1}}+\cdots+\tilde{\gamma}_{i_{d}}$ belongs to the logarithmic jacobian ideal $\tilde{\mathcal{J}}$, which shows that the image of $\mathcal{J}$ is equal to $\tilde{\mathcal{J}}$.

\section{ITERATING THE BLOWING-UP OF THE LOGARITHMIC JACOBIAN IDEAL}

Let $\Gamma \subset M$ a finitely generated subsemigroup of a rank $d$ lattice $M$ such that $\mathbf{Z} \Gamma=M$. We assume in addition that the convex rational cone $\check{\sigma}:=\mathbf{R}_{>0} \Gamma$, which is $d$-dimensional since $\mathbf{Z} \Gamma=M$, is strictly convex, which is equivalent to saying that the dual cone $\sigma \subset N_{\mathbf{R}}$ is strictly convex of dimension $d$. The semigroup $\Gamma$ determines the affine toric variety $T^{\Gamma}=\operatorname{Speck}\left[t^{\Gamma}\right]$. We fix a finite set of generators $\gamma_{1}, \ldots, \gamma_{r}$ of $\Gamma$. We consider the set

$$
\mathcal{J}:=\left\{\gamma_{i_{1}}+\cdots+\gamma_{i_{d}} \mid \gamma_{i_{1}} \wedge \ldots \wedge \gamma_{i_{d}} \neq 0,1 \leq i_{1}, \ldots, i_{d} \leq r\right\}
$$

defining the logarithmic jacobian ideal of $T^{\Gamma}$.

The Newton polyhedron $\mathcal{N}_{\sigma}(\mathcal{J})$ of the monomial ideal $\mathcal{J}$ (see Section 5 ), is contained in the interior of $\check{\sigma}$, since the elements of $\mathcal{J}$ are sums of $d$-linearly independent elements in the $d$-dimensional cone $\check{\sigma}$. The set $\mathcal{J}$ determines the order function defined by (3). The maximal cones $\tau \subset \sigma$ of linearity of the function ord $\mathcal{J}$ form the $d$-skeleton of a fan $\Sigma$ supported on $\sigma$. The map

$$
\tau \mapsto m \text { if } \operatorname{ord}_{\mathcal{J}}(\nu)=\langle\nu, m\rangle \text { for all } \nu \in \tau .
$$

is a bijection between the set $\Sigma(d)$ of $d$-dimensional cones of $\Sigma$ and the set of vertices of the polyhedron $\mathcal{N}_{\sigma}(\mathcal{J})$. 
We now consider the blowing up of the monomial ideal $\mathcal{J}$. A cone $\tau^{(1)} \in \Sigma(d)$ determines a vertex $m^{(1)}$ of $\mathcal{N}_{\sigma}(\mathcal{J})$ by (18) and also the finitely generated semigroup

$$
\Gamma_{\tau^{(1)}}^{(2)}:=\Gamma+\sum_{m \in \mathcal{J}} \mathbf{Z}_{\geq 0}\left(m-m^{(1)}\right) \subset \check{\tau}^{(1)} \cap M .
$$

In view of the description recalled above of $\Sigma(d)$ in terms of $\mathcal{N}_{\sigma}(\mathcal{J})$, the cone $\mathbf{R}_{\geq 0} \Gamma_{\tau^{(1)}}^{(2)}$ is $\check{\tau}^{(1)}$. The affine toric variety $T_{\tau^{(1)}}^{\Gamma^{(2)}}$ is a chart of the blowing up of $\mathcal{J}$ and this toric variety is covered by charts of this form (see Section 5 ). The semigroup $\Gamma_{\tau^{(1)}}^{(2)}$ is generated by $\left\{\gamma_{1}, \ldots, \gamma_{r}\right\} \cup\left\{m-m^{(1)}\right\}_{m \in \mathcal{J}}$.

Lemma 12.1. If we choose a representation $m^{(1)}=\gamma_{i_{1}}+\cdots+\gamma_{i_{d}}$ as a sum of linearly independent vectors in $\Gamma$ then the semigroup $\Gamma_{\tau^{(1)}}^{(2)}$ is generated by

$$
\left\{\gamma_{i_{1}}, \ldots, \gamma_{i_{d}}\right\} \cup\left\{\gamma_{l}-\gamma_{i_{s}} \mid 1 \leq s \leq d, 1 \leq l \leq r, l \neq i_{s}, \gamma_{l} \wedge \bigwedge_{j=1, \ldots, d}^{j \neq i_{s}} \gamma_{i_{j}} \neq 0\right\} .
$$

Proof. To simplify the notations we can assume that $i_{s}=s$ for $s=1, \ldots, d$. First notice that if $\gamma_{l}, 1 \leq l \leq r$, has the property that $\gamma_{l} \wedge \bigwedge_{i=1, \ldots, d}^{i \neq i_{0}} \gamma_{i}$ then the vector $m^{\prime}=\gamma_{l}+\sum_{i=1, \ldots, d}^{i \neq i_{0}} \gamma_{i}$ belongs to $\mathcal{J}$ hence $m^{\prime}-m^{(1)}=\gamma_{l}-\gamma_{i_{0}}$ is a generator of $\Gamma_{\tau^{(1)}}^{(2)}$. Since $\gamma_{1}, \ldots, \gamma_{d} \in \Gamma_{\tau^{(1)}}^{(2)}$ by construction we get that $\gamma_{l} \in \Gamma_{\tau^{(1)}}^{(2)}$ is not in the minimal set of generators of the semigroup $\Gamma_{\tau^{(1)}}^{(2)}$.

Suppose now that $\gamma_{p_{1}}, \ldots, \gamma_{p_{d}}$ are linearly independent vectors. We denote by $s_{0}$ the integer such that $\gamma_{p_{1}}, \ldots, \gamma_{p_{s_{0}}} \in\{1, \ldots, d\}$ and $\gamma_{p_{s_{0}+1}}, \ldots, \gamma_{p_{d}} \in\{d+1, \ldots, r\}$. We can permute the vectors $\gamma_{p_{s_{0}+1}}, \ldots, \gamma_{p_{d}}$ in such a way that the $i$-th coefficient of the expansion of $\gamma_{p_{i}}$ in terms of the basis $\gamma_{1}, \ldots, \gamma_{d}$ of $N_{\mathbf{Q}}$, is non-zero for $i=s_{0}+1, \ldots, d$ (otherwise we would get that $\gamma_{p_{1}} \wedge \cdots \wedge \gamma_{p_{d}}=0$ contrary to the assumption). Then the vector $m=\sum_{i=1}^{d} \gamma_{p_{i}}$ belongs to $\mathcal{J}$ and we deduce that $m-m^{(1)}=\sum_{i=s_{0}+1}^{d}\left(\gamma_{p_{i}}-\gamma_{i}\right)$ hence the vectors (19) generate the semigroup $\Gamma_{\tau^{(1)}}^{(2)}$.

We denote also by $\mathcal{J}_{\tau^{(1)}}^{(2)}$ the finite subset of $\Gamma_{\tau^{(1)}}^{(2)}$ corresponding to the monomials generating the logarithmic jacobian ideal of $T_{\tau^{(1)}}^{\Gamma^{(2)}}$, by the same symbol this last ideal of $k\left[t_{\tau^{(1)}}^{\Gamma^{(2)}}\right]$, and by ord $\mathcal{J}_{\tau^{(1)}}^{(2)}: \tau^{(1)} \rightarrow \mathbf{R}$ the corresponding order function.

Remark 12.2. On the chart $T_{\tau^{(1)}}^{\Gamma^{(2)}}$ the pull back of the ideal $\mathcal{J}$ by the blowing up of $\mathcal{J}$ is the principal ideal $t^{m^{(1)}} k\left[t_{\tau^{(1)}}^{\Gamma^{(2)}}\right]=t^{\mathcal{J}} k\left[t_{\tau^{(1)}}^{\Gamma^{(2)}}\right]$. The Newton polyhedron

$$
\mathcal{N}_{\tau^{(1)}}(\mathcal{J}):=\mathcal{J}+\check{\tau}^{(1)}=m^{(1)}+\check{\tau}^{(1)}
$$

of $t^{\mathcal{J}} k\left[t_{\tau^{(1)}}^{\Gamma^{(2)}}\right]$ is principal, i.e., it has only one vertex $m^{(1)}$.

Lemma 12.3. There is a continuous piecewise linear function $\operatorname{ord}_{\mathcal{J}^{(2)}}: \sigma \rightarrow \mathbf{R}$ such that for each $\tau^{(1)} \in \Sigma(d)$ the function ord $_{\mathcal{J}_{\tau^{(1)}}^{(2)}}$ is the restriction of ord $\mathcal{J}^{(2)}$ to $\tau^{(1)}$.

Proof. This follows from Lemma 11.4.

As above, the maximal cones $\tau \subset \sigma$ of linearity of the function ord $\mathcal{J}^{(2)}$ form the $d$-skeleton of a fan $\Sigma^{(2)}$ supported on $\sigma$ and subdividing the fan $\Sigma$. In particular, if 
$\tau^{(2)} \in \Sigma^{(2)}(d)$ is contained in $\tau^{(1)} \in \Sigma(d)$ then we denote by $m^{(2)}$ the vertex of the Newton polyhedron $\mathcal{N}_{\tau^{(1)}}\left(\mathcal{J}_{\tau^{(1)}}^{(2)}\right)$ of $\mathcal{J}_{\tau^{(1)}}^{(2)}$ such that

$$
\operatorname{ord}_{\mathcal{J}^{(2)}}(\nu)=\left\langle\nu, m^{(2)}\right\rangle \text { for all } \nu \in \tau^{(2)} \text {. }
$$

By iterating this construction we obtain a sequence of piecewise linear functions $\operatorname{ord}_{\mathcal{J}^{(j)}}$ on $\sigma$, together with the corresponding fans $\Sigma^{(j)}$, with $\mathcal{J}=\mathcal{J}^{(1)}$ and $\Sigma^{(1)}=\Sigma$, and such that $\Sigma^{(j)}$ is a subdivision of $\Sigma^{(j-1)}$ for all $j \geq 2$.

By definition a cone $\tau^{(j)} \in \Sigma^{(j)}(d)$ is contained in a unique cone $\tau^{(l)} \in \Sigma^{(l)}(d)$, for $0 \leq l \leq j-1$, where we set $\tau^{(0)}:=\sigma$. Then we have unique vectors $m^{(l)} \in M$ such that

$$
\operatorname{ord}_{\mathcal{J}^{(l)}}(\nu)=\left\langle\nu, m^{(l)}\right\rangle \text { for all } \nu \in \tau^{(j)} \text { and } 1 \leq l \leq j .
$$

The cone $\tau^{(j)}$ corresponds to a chart of the blowing up of the logarithmic jacobian ideal $\mathcal{J}_{\tau^{(j-1)}}^{(j)}$ of $k\left[t_{\tau^{(j-1)}}^{\Gamma^{(j)}}\right]$. This chart is the affine toric variety defined by the semigroup

$$
\Gamma_{\tau^{(j)}}^{(j+1)}=\Gamma_{\tau^{(j-1)}}^{(j)}+\sum_{m \in \mathcal{J}_{\tau^{(j-1)}}^{(j)}} \mathbf{Z}_{\geq 0}\left(m-m^{(j)}\right) .
$$

By induction this procedure also provides a system of generators of each semigroup $\Gamma_{\tau^{(j)}}^{(j+1)}$. We use also the notation $\mathcal{J}_{\tau^{(j)}}^{(j+1)}$ to refer to the finite set of generators of the logarithmic jacobian ideal of $k\left[t_{\tau}^{\Gamma_{\tau}^{(j)}}\right]$ (see Remark 11.1). The following inclusions, for $j \geq 2$, are consequence of the definitions:

$$
\Gamma_{\tau^{(j-1)}}^{(j)} \subset \Gamma_{\tau^{(j)}}^{(j+1)}, \quad k\left[t_{\tau^{(j-1)}}^{\Gamma^{(j)}}\right] \subset k\left[t_{\tau^{(j)}}^{\Gamma^{(j+1)}}\right], \quad \mathcal{J}_{\tau^{(j-1)}}^{(j)} k\left[t_{\tau^{(j)}}^{\Gamma^{(j+1)}}\right] \subset \mathcal{J}_{\tau^{(j)}}^{(j+1)} k\left[t_{\tau^{(j)}}^{\Gamma^{(j+1)}}\right] .
$$

By (20) we have that

$$
\operatorname{ord}_{\mathcal{J}^{(j+1)}}(\nu) \leq \operatorname{ord}_{\mathcal{J}^{(j)}}(\nu) \text { for all } \nu \in \sigma \text {. }
$$

Remark 12.4. For $1 \leq l \leq j$ we deduce from Remark 12.2 that $\mathcal{J}_{\tau^{(l-1)}}^{(l)} k\left[t_{\tau^{\Gamma^{(l)}}}^{(l+1)}\right]=$ $t^{m^{(l)}} k\left[t_{\tau^{(l)}}^{\Gamma^{(l+1)}}\right]$, hence the Newton polyhedron $\mathcal{N}_{\tau^{(l)}}\left(\mathcal{J}_{\tau^{(l-1)}}^{(l)}\right)=\mathcal{J}_{\tau^{(l-1)}}^{(l)}+\check{\tau}^{(l)}=m^{(l)}+\check{\tau}^{(l)}$ has only one vertex $m^{(l)}$.

Notation 12.5. We denote the Newton polyhedron $\mathcal{N}_{\tau^{(j-1)}}\left(\mathcal{J}_{\tau^{(j-1)}}^{(j)}\right) \operatorname{simply}$ by $\mathcal{N}\left(\mathcal{J}_{\tau^{(j-1)}}^{(j)}\right)$ since there is no risk of confusion.

Proposition 12.6. The following assertions are equivalent:

i. $\tau^{(j)}=\tau^{(j-1)}$

ii. The blowing up of the ideal $\mathcal{J}_{\tau^{(j-1)}}^{(j)}$ of $T_{\tau^{\Gamma^{(j-1)}}}^{(j)}$ is a finite morphism.

Proof. The hypothesis i. is equivalent to the following fact: the semigroups $\Gamma_{\tau^{(j-1)}}^{(j)}$ and $\Gamma_{\tau^{(j)}}^{(j+1)}$ have the same saturation in the lattice $M$; it is equal to $\check{\tau}^{(j-1)} \cap M=$ $\check{\tau}^{(j)} \cap M$. This is equivalent to the following geometric statement: the composite of the normalization of $T_{\tau^{(j)}}^{\Gamma^{(j+1)}}$ with the blowing up of the logarithmic jacobian ideal of $T_{\tau^{(j-1)}}^{\Gamma^{(j)}}$ is the normalization map of $T_{\tau^{(j-1)}}^{\Gamma^{(j)}}$ and therefore this blowing up is finite. Conversely, if ii. holds, the blowing up morphism $T_{\tau^{(j)}}^{\Gamma^{(j+1)}} \rightarrow T_{\tau^{(j-1)}}^{\Gamma^{(j)}}$ induces an isomorphism of the normalizations, from which i. follows in view of Remark 4.6.

Remark 12.7. The conditions of the Lemma are also equivalent to the fact that the Newton polyhedron of the ideal $\mathcal{J}_{\tau^{(j-1)}}^{(j)}$ has only one vertex $m^{(j)}$. 
Definition 12.8. For any integer $j \geq 1$ we introduce a function

$$
f^{(j)}:\{\tau \subset \sigma \mid 0 \neq \tau \text { convex rational polyhedral cone }\} \rightarrow \mathbf{Z}_{\geq 1} \text {. }
$$

If $\nu_{1}, \ldots, \nu_{s}$ are the primitive integral vectors for the lattice $N$ which span the edges of $\tau$, then the value of $f^{(j)}(\tau)$ is defined by

$$
f^{(j)}(\tau):=\sum_{i=1}^{s} \operatorname{ord}_{\mathcal{J}^{(j)}}\left(\nu_{i}\right) .
$$

Remark 12.9. Notice that if $0 \neq \tau$ is any rational polyhedral cone contained in $\tau^{(j)} \in$ $\Sigma^{(j)}(d)$ then $f^{(j)}(\tau)=\sum_{i=1}^{s}\left\langle\nu_{i}, m^{(j)}\right\rangle$ and if $\tau$ is of dimension $d$ then $f^{(j)}(\tau) \geq d$. Moreover, by (21) we obtain that

$$
f^{(j)}(\tau) \leq f^{(j-1)}(\tau)
$$

Lemma 12.10. The following conditions are equivalent for $j \geq 1$ :

i. The equality $f^{(j)}\left(\tau^{(j-1)}\right)=d$ holds.

ii. The cone $\tau^{(j-1)}$ is regular for the lattice $N$ and $\Gamma_{\tau^{(j-1)}}^{(j)}=\check{\tau}^{(j-1)} \cap M$.

iii. The toric variety $T_{\tau_{\tau}^{\Gamma^{(j-1)}}}^{(j)}$ is smooth.

Note that if the conditions of the Lemma are satisfied, the polyhedron $\mathcal{N}_{\tau^{(j-1)}}\left(\mathcal{J}_{\tau^{(j-1)}}^{(j)}\right)$ has only one vertex $m^{(j)}$.

Proof. It is clear that ii. and iii. are equivalent. It is enough to prove the result for $j=1$. Suppose first that i. holds. By hypothesis the fan $\Sigma^{(1)}$ is the fan consisting of the faces of $\sigma$. If $\nu_{1}, \ldots, \nu_{s}$ are the primitive vectors for the lattice $N$ which span the cone $\sigma$ then $\left\langle\nu_{i}, m\right\rangle>0, i=1, \ldots, s$ since $m=m^{(1)}$ belongs to the interior of $\check{\sigma}$. Since $f(\sigma)=d=\sum_{i=1}^{s}\left\langle\nu_{i}, m\right\rangle$ we get that $s=d$ and $\left\langle\nu_{i}, m\right\rangle=1$.

By definition of $\mathcal{J}$ the vector $m$ is sum of $d$ generators of $\Gamma$ which are linearly independent, say $m=\gamma_{1}+\cdots+\gamma_{d}$. Since $\sum_{j=1}^{d}\left\langle\nu_{i}, \gamma_{j}\right\rangle=1$ for $i=1, \ldots, d$ we obtain that, up to relabeling the $\nu_{i}$, the vectors $\nu_{1}, \ldots, \nu_{d}$ in $N_{\mathbf{R}}$ form the dual basis of $\gamma_{1}, \ldots, \gamma_{d}$ in $M_{\mathbf{R}}$. Finally, notice that the parallelogram generated by the primitive vectors $\gamma_{1}, \ldots, \gamma_{d}$ in $M_{\mathbf{R}}$ contains no integral points different from the vertices. It follows that $\gamma_{1}, \ldots, \gamma_{d}$ form a basis of $M$.

Conversely, if ii. holds then we check from the definitions that i. holds.

Proposition 12.11. Suppose that $\tau^{(j)} \in \Sigma^{(j)}(d)$ is contained in $\tau^{(j-1)} \in \Sigma^{(j-1)}(d)$. The following equalities are equivalent:
i. $f^{(j)}\left(\tau^{(j)}\right)=f^{(j-1)}\left(\tau^{(j)}\right)$,
ii. $m^{(j)}=m^{(j-1)}$.

Proof. Notice that if $m^{(j)}=m^{(j-1)}$ then i. follows by Remark 12.9

Suppose that the equality i. holds. By Remark 12.4 we have that

$$
\mathcal{N}_{\tau^{(j-1)}}\left(\mathcal{J}_{\tau^{(j-2)}}^{(j-1)}\right)=m^{(j-1)}+\check{\tau}^{(j-1)} \text { and } \mathcal{N}_{\tau^{(j)}}\left(\mathcal{J}_{\tau^{(j-1)}}^{(j)}\right)=m^{(j)}+\check{\tau}^{(j)} .
$$

Since $\tau^{(j)}$ is contained in $\tau^{(j-1)}$ we get that $\check{\tau}^{(j-1)} \subset \check{\tau}^{(j)}$ and then $\mathcal{N}_{\tau^{(j)}}\left(\mathcal{J}_{\tau^{(j-2)}}^{(j-1)}\right)=$ $m^{(j-1)}+\check{\tau}^{(j)}$. By (20) we get

$$
m^{(j-1)}+\check{\tau}^{(j)} \subset m^{(j)}+\check{\tau}^{(j)} .
$$

Let $\nu_{1}, \ldots, \nu_{s}$ be the primitive integral vectors for the lattice $N$ which span the cone $\tau^{(j)}$. The vector $\nu:=\sum_{i=1}^{s} \nu_{i}$ belongs to the interior of the cones $\tau^{(j)}$ and $\tau^{(j-1)}$. By Remark 12.9 and the hypothesis we deduce

$$
f^{(j-1)}\left(\tau^{(j)}\right)=\left\langle\nu, m^{(j-1)}\right\rangle=f^{(j)}\left(\tau^{(j)}\right)=\left\langle\nu, m^{(j)}\right\rangle .
$$


This equality and the inclusion (23) imply that $m^{(j-1)}=m^{(j)}$.

Proposition 12.12. There exists an integer $l \geq 1$ such that for any cone $\tau \in \Sigma^{(l)}(d)$ if $f^{(1)}(\tau)>d$ then $f^{(1)}(\tau)>f^{(l)}(\tau)$.

Proof. Let us assume that the assertion of the Proposition does not hold. This implies that there exists a infinite sequence of convex polyhedral cones

$$
\sigma=\tau^{(0)} \supset \tau^{(1)} \supseteq \tau^{(2)} \supseteq \cdots \supseteq \tau^{(j)} \supseteq \cdots,
$$

such that $\tau^{(j)} \in \Sigma^{(j)}(d)$ and

$$
f^{(j)}\left(\tau^{(j)}\right)=f^{(1)}\left(\tau^{(j)}\right)>d, \text { for all } j \geq 2 .
$$

By Remark 12.9 we have that $f^{(j)}\left(\tau^{(j)}\right)=f^{(j-1)}\left(\tau^{(j)}\right)$ for all $j \geq 2$. Proposition 12.11 implies then that $m^{(j)}=m^{(j-1)}$ for all $j \geq 2$.

Claim 12.13. There exists a strictly increasing sequence $\left(i_{j}\right)_{j \geq 1}$ of integers $\geq 0$ such that $\tau^{\left(i_{j}\right)} \neq \tau^{\left(i_{j}+1\right)}$, that is, the inclusion $\tau^{\left(i_{j}\right)} \supset \tau^{\left(i_{j}+1\right)}$ is strict, for $j \geq 1$.

Proof of the claim. Assume that the claim does not hold. This implies that $\tau^{(j)}=$ $\tau^{(j-1)}$ for all $j \geq 1$. By Proposition 12.6 the blowing up of the ideal $\mathcal{J}_{\tau^{(j-1)}}^{(j)}$ of $T^{\Gamma_{\tau^{(j-1)}}^{(j)}}$ is a finite morphism, dominated by the normalization of $T^{\Gamma}$, for all $j \geq 1$. It follows that for $j \gg 0$ the variety $T_{\tau^{(j-1)}}^{\Gamma^{(j)}}$ is normal. By Proposition 11.3, this variety is also smooth. By Lemma12.10 it follows that $f^{(j)}\left(\tau^{(j)}\right)=d$ for $j \gg 0$. This is a contradiction with (25).

Let us fix a representation for $m=m^{(1)}$ in terms of the generators of $\Gamma$ :

$$
m=\gamma_{1}+\cdots+\gamma_{d} \text { with } \gamma_{1} \wedge \cdots \wedge \gamma_{d} \neq 0,
$$

(up to an eventual relabeling of the generators $\left\{\gamma_{i}\right\}_{i=1}^{r}$ of the semigroup $\Gamma$ ).

By Claim 12.13 we can suppose without loss of generality that $i_{1}=0$, that is, the Newton polyhedron $\mathcal{N}_{\sigma}(\mathcal{J})$ has at least two different vertices $m$ and $n$.

Lemma 12.14. Given one of the $\gamma_{j}$ which appear in the decomposition of $m$, say $\gamma_{d}$, for any $j \geq 0$ the vector $n_{j}:=n-j \gamma_{d}$ has the property that

$$
n_{j} \in \mathcal{J}_{\tau^{(j)}}^{(j+1)} \text { and }\left(n_{j}-m\right) \wedge \gamma_{1} \wedge \cdots \wedge \gamma_{d-1} \neq 0 .
$$

Proof. We prove the assertion by induction on $j$. Notice that for $j=0$ the vector $n_{0}=n$ belongs to $\mathcal{J}$ by hypothesis. We suppose by induction that $n_{l} \in \mathcal{J}_{\tau^{(l)}}^{(l+1)}$, $1 \leq l \leq j$

We prove first that:

$$
\left(n_{l}-m\right) \wedge \gamma_{1} \wedge \cdots \wedge \gamma_{d-1} \neq 0, \text { for } 0 \leq l \leq j .
$$

Assume on the contrary that (26) does not hold for some $0 \leq l \leq j$. After relabeling the vectors $\gamma_{1}, \ldots, \gamma_{d-1}$ if necessary, we have an expansion of the form:

$$
n_{l}-m=a_{1} \gamma_{1}+\cdots+a_{h} \gamma_{h} \text { with } h \leq d-1,
$$

and in addition the coefficients of (27) are non-zero rational numbers which are not of the same sign, that is,

$$
\left\{\begin{array}{l}
a_{i}>0 \text { for } i=1, \ldots, s \\
a_{i}<0 \text { for } i=s+1, \ldots, h .
\end{array}\right.
$$

Indeed, if all coefficients $a_{i}$ in (27) are $\geq 0$ we obtain that

$$
n=m+a_{1} \gamma_{1}+\cdots+a_{h} \gamma_{h}+l \gamma_{d} \subset m+\check{\sigma},
$$


contradicting that $n \neq m$ is a vertex of $\mathcal{N}_{\sigma}(\mathcal{J})$. In particular, we have that $n_{l} \neq m$. Similarly, if all the coefficients $a_{i}$ are smaller than zero we get that

$$
m=n_{l}-a_{1} \gamma_{1}-\cdots-a_{h} \gamma_{h} \subset n_{l}+\check{\sigma} \subset n_{l}+\check{\tau}^{(l)} .
$$

This implies that $m$ is not a vertex of the Newton polyhedron $\mathcal{N}_{\tau^{(l)}}\left(\mathcal{J}_{\tau^{(l)}}^{(l+1)}\right)$, since $n_{l} \in \mathcal{J}_{\tau^{(l)}}^{(l+1)}$ and $n_{l} \neq m$.

If $\alpha \in \mathbf{R}$ we denote by $\lceil\alpha\rceil$ the smallest integer $p$ such that $\alpha \leq p$.

Claim 12.15. If $q:=\sum_{i=1}^{s}\left\lceil a_{i}\right\rceil, 0 \leq p \leq q$ and $b_{i}$ are integers such that $0 \leq b_{i} \leq\left\lceil a_{i}\right\rceil$, $i=1, \ldots, s$ and $\sum_{i=1}^{s} b_{i}=p$ then the vector $\beta_{l, p}:=n_{l}-\sum_{i=1}^{s} b_{i} \gamma_{i}$ belongs to $\mathcal{J}_{\tau^{(l+p)}}^{(l+p+1)}$.

Proof of the claim. We prove the assertion by induction on $p$. For $p=0$ we have $\beta_{l, 0}=n_{l}$ hence the assertion holds by assumption. Suppose that $\beta_{l, p} \in \mathcal{J}_{\tau^{(l+p)}}^{(l+p+1)}$ for $0 \leq p<q$. The vector

$$
\beta_{l, p}-m=n_{l}-m-\sum_{i=1}^{s} b_{i} \gamma_{i}=\sum_{i=1}^{s}\left(a_{i}-b_{i}\right) \gamma_{i}+\sum_{i=s+1}^{h} a_{i} \gamma_{i}
$$

belongs to $\Gamma_{\tau^{(l+p+1)}}^{(l+p+2)}$. Since $p<q$ there is a strictly positive coefficient in this expansion of $\beta_{l, p}-m$, say $a_{1}-b_{1}$, for instance. We get $\left(\beta_{l, p}-m\right) \wedge \gamma_{2} \wedge \cdots \wedge \gamma_{d} \neq 0$, hence the vector

$$
\beta_{l, p+1}:=\beta_{l, p}-m+\gamma_{2}+\cdots+\gamma_{d}=\beta_{l, p}-\gamma_{1}
$$

belongs to $\mathcal{J}_{\tau^{(l+p+1)}}^{(l+p+2)}$.

By Claim 12.15 the expansion

$$
\beta_{l, q}-m=\sum_{i=1}^{s}\left(a_{i}-\left\lceil a_{i}\right\rceil\right) \gamma_{i}+\sum_{i=s+1}^{h} a_{i} \gamma_{i}
$$

has only coefficients $\leq 0$ and $\beta_{l, q} \in \mathcal{J}_{\tau^{(l+q+1)}}^{(l+q+2)}$. We get also that $m \neq \beta_{l, q}$ since the coefficients $a_{s+1}, \ldots, a_{h}$ are non-zero and the vectors $\gamma_{1}, \ldots, \gamma_{h}$ are linearly independent. We deduce from this that

$$
m=\beta_{l, q}-\sum_{i=1}^{s}\left(a_{i}-\left\lceil a_{i}\right\rceil\right) \gamma_{i}-\sum_{i=s+1}^{h} a_{i} \gamma_{i} \in \beta_{l, q}+\check{\sigma} \subset \beta_{l, q}+\check{\tau}^{(j+q+1)} .
$$

This contradicts the assumption, $m$ being a vertex of the Newton polyhedron of $\mathcal{J}_{\tau^{(l+q+1)}}^{(l+q+2)}$.

Finally, we have proven that (26) holds hence

$$
n_{j+1}=\left(n_{j}-m\right)+\gamma_{1}+\cdots+\gamma_{d-1} \in \mathcal{J}_{\tau^{(j+1)}}^{(j+2)} .
$$

This concludes the induction in the proof of Lemma 12.14.

The cone

$$
\tau^{(\infty)}=\bigcap_{l \geq 1} \tau^{(l)}=\bigcap_{j \geq 1} \tau^{\left(i_{j}\right)}
$$

is a closed convex subset of $\sigma$ different from 0 . A vector $0 \neq w \in \tau^{(\infty)}$ defines a monomial valuation $\omega$ of the field of fractions of $k\left[t^{\Gamma}\right]$, which verifies that if $0 \neq \sum a_{\gamma} t^{\gamma} \in k\left[t^{\Gamma}\right]$ then $\omega\left(\sum a_{\gamma} t^{\gamma}\right)=\min _{a_{\gamma} \neq 0}\langle w, \gamma\rangle$. By definition this valuation is non-negative in the subrings $k\left[t_{\tau}^{\Gamma_{\tau}^{(j)}(j-1)}\right]$ for all $j \geq 1$. Notice that the vector $w \in N_{\mathbf{R}}$ is not necessarily an element of $N_{\mathbf{Q}}$ and it may lie in a face of $\sigma$ (different from 0 ). We remark that for all 
$j \geq 1$ we have that $\min \left\{\langle w, \gamma\rangle \mid \gamma \in \mathcal{J}_{\tau^{(j-1)}}^{(j)}\right\}=\langle w, m\rangle$ since $w$ takes non negative values on $\mathcal{J}_{\tau^{(j)}}^{(j+1)}$ which contains the set $\left\{\gamma-m \mid \gamma \in \mathcal{J}_{\tau^{(j-1)}}^{(j)}\right\}$.

Since $\gamma_{1} \ldots, \gamma_{d}$ span $M_{\mathbf{R}}$ at least one of the vectors $\gamma_{i}$ verifies that $\left\langle w, \gamma_{i}\right\rangle \neq 0$. Suppose for instance that $\left\langle w, \gamma_{d}\right\rangle>0$.

By Lemma 12.14, for any integer $j \geq 0$ the vector $n_{j}=n-j \gamma_{d}$ belongs to $\mathcal{J}_{\tau^{(j)}}^{(j+1)} \subset$ $\Gamma_{\tau^{(j)}}^{(j+1)}$. This implies that

$$
\omega\left(t^{n_{j}}\right)=\left\langle w, n_{j}\right\rangle=\langle w, n\rangle-j\left\langle w, \gamma_{d}\right\rangle
$$

becomes strictly negative for $j$ large enough. This is a contradiction since $t^{n_{j}} \in k\left[t_{\tau^{(j)}}^{\Gamma^{(j+1)}}\right]$ and the valuation $\omega$ is non negative on the ring $k\left[t_{\tau^{(j)}}^{\Gamma^{(j+1)}}\right]$.

Corollary 12.16. With the previous notations, given any sequence of the form 24) if $T^{\Gamma}$ is not smooth it is not possible that $m^{(1)}=m^{(j)}$ for all $j \geq 2$.

Proof. This is now a consequence of Proposition 12.11.

\section{Definition 12.17.}

i. If $0 \neq \eta \subset \sigma$ is a cone we denote by $\nu_{\eta}$ the sum of the primitive vectors, for the lattice $N$ in the edges of the cone $\eta$.

ii. A cone $\eta \subset \sigma$ is stable if there is an integer $I \geq 1$ such that $\eta \in \Sigma^{(j)}$ for all $j \geq I$.

iii. The stability problem for the toric variety $T^{\Gamma}$ consists of determining if the sequence of fans $\left(\Sigma^{(j)}\right)_{j \geq 0}$ stabilizes, that is $\Sigma^{(j)}=\Sigma^{(j+1)}$ for $j \gg 0$.

iv. If $\theta \in \Sigma^{(l)}$ the stability problem for the cone $\theta$ consists of determining if the sequence of fans, $\left\{\theta^{(j)} \in \Sigma^{(j)} \mid \theta^{(j)} \subset \theta\right\} j \geq l$, stabilizes.

For instance, if $\rho \in \Sigma^{(j)}$ is of dimension one then $\rho$ is stable.

Lemma 12.18. If $\theta \in \Sigma^{(j-1)}$ is a cone of codimension $>0$ and $M\left(\theta, \Gamma_{\theta}^{(j)}\right)=M(\theta)$ (see Notation 4.9), then the stability problem for the cone $\theta \subset N_{\mathbf{R}}$ is equivalent to a stability problem for the cone $\theta$, viewed in $\left(N_{\theta}\right)_{\mathbf{R}}$, with respect to the sequence of iterated Semple-Nash modifications of another toric variety of dimension equal to $\operatorname{dim} \theta$.

Proof. The sublattice $M(\theta)=M \cap \theta^{\perp}$ of $M$ is obviously saturated, so that it is a direct summand of $M$. Let us consider a sublattice $M^{\prime}$ of $M$ such that $M=M(\theta) \oplus M^{\prime}$. Such a sublattice $M^{\prime}$ is spanned by vectors $v_{q_{0}+1}, \ldots, v_{d}$, completing a basis $v_{1}, \ldots, v_{q_{0}}$ of $M(\theta)$ to a basis of $M$. Any $\gamma \in M$ can be written in a unique way as $\gamma=\alpha_{1}(\gamma)+\alpha_{2}(\gamma)$ with $\alpha_{1}(\gamma) \in M(\theta)$ and $\alpha_{2}(\gamma) \in M^{\prime}$. The restriction $\beta: M^{\prime} \mapsto M / M(\theta)$ of the canonical map $M \rightarrow M / M(\theta)$ to the sublattice $M^{\prime} \subset M$ is an isomorphism. The image $\tilde{\Gamma}_{\theta}^{(j)}$ of $\Gamma_{\theta}^{(j)}$ by the canonical map $M \mapsto M / M(\theta)$ is a semigroup of finite type, generates the lattice $M / M(\theta)$ and spans a strictly convex cone $\mathbf{R}_{\geq 0} \tilde{\Gamma}_{\theta}^{(j)}$.

The semigroup $\Gamma_{\theta}^{(j)} \cap \theta^{\perp}$, which is the minimal face of $\Gamma_{\theta}^{(j)}$, is a rank $q_{0}$ lattice by Lemma 3.9. Using the equality $\Gamma_{\theta}^{(j)} \cap \theta^{\perp}=M(\theta)$ one checks directly that the map

$$
\Gamma_{\theta}^{(j)} \longrightarrow M(\theta) \times \tilde{\Gamma}_{\theta}^{(j)}, \quad \gamma \mapsto\left(\alpha_{1}(\gamma), \beta \circ \alpha_{2}(\gamma)\right)
$$

is a semigroup isomorphism. The map (28) determines an isomorphism $T^{\Gamma_{\theta}^{(j)}} \stackrel{\simeq}{\longrightarrow}$ $\operatorname{orb}\left(\theta, \Gamma_{\theta}^{(j)}\right) \times T^{\tilde{\Gamma}_{\theta}^{(j)}}$. In particular, the variety $T^{\Gamma_{\theta}^{(j)}}$ is smooth if and only if $T^{\tilde{\Gamma}_{\theta}^{(j)}}$ is smooth.

According to Remark 10.3 and Lemma 11.6 the blowing up of logarithmic jacobian ideals commutes with the splitting defined by (28). The assertion follows from this since 
the cone $\theta$, viewed in the $\mathbf{R}$-linear subspace $\left(N_{\theta}\right)_{\mathbf{R}}$ it spans in $N_{\mathbf{R}}$, is the dual cone of $\mathbf{R}_{\geq 0} \tilde{\Gamma}_{\theta}^{(j)}$.

Remark 12.19. Geometrically, we see that the semigroup $\tilde{\Gamma}_{\theta}^{(j)}$ in Lemma 12.18 corresponds to the toric variety of dimension $\operatorname{dim} \theta$ which is a transverse linear section of $T^{\Gamma_{\theta}^{(j)}}$ at the point $(1, \ldots, 1)$ of the orbit corresponding to $\theta$.

Proposition 12.20. If $\theta$ is a stable cone then there is an integer $I \geq 1$ such that the variety $T^{\Gamma_{\theta}^{(j)}}$ is smooth for all $j \geq$ I. If $T^{\Gamma_{\theta}^{(j)}}$ is smooth the cone $\theta$ is stable.

Proof. By Lemma 12.18 and ii. of Proposition 12.22 below, we can assume that $\operatorname{codim} \theta=0$. The blowing up of the ideal $\mathcal{J}_{\theta}^{(j)}$ of $T^{\Gamma_{\theta}^{(j)}}$ is a finite morphism, dominated for all $j \gg 0$ by the normalization of $T^{\Gamma_{\theta}^{(j)}}$, which is equal to that of $T^{\Gamma_{\theta}^{(1)}}$ (see Proposition 12.6). It follows that for $j \gg 0$ the map $T^{\Gamma_{\theta}^{(j+1)}} \rightarrow T^{\Gamma_{\theta}^{(j)}}$ is an isomorphism. By Proposition 11.3, this variety is smooth. The second assertion follows directly from the definitions.

Remark 12.21. By Proposition12.20 the stability problem is equivalent to the problem of determining if the iteration of the blowing ups of logarithmic jacobian ideals eventually resolves the singularities of the toric variety $T^{\Gamma}$.

Proposition 12.22. Given $0 \neq \nu \in \sigma \cap N$, for any $j \geq 1$ there exists a unique cone $\theta^{(j)} \in \Sigma^{(j)}$ such that $\nu \in \operatorname{int}\left(\theta^{(j)}\right)$. Then for $j \gg 0$ we have:

i. If $q_{0}=\operatorname{codim} \theta^{(j-1)}$ and if $m^{(j)} \in \mathcal{J}_{\theta^{(j-1)}}^{(j)}$ is such that $\operatorname{ord}_{\mathcal{J}^{(j)}}(\nu)=\left\langle\nu, m^{(j)}\right\rangle$, then for any representation

$$
m^{(j)}=\gamma_{1}^{(j)}+\cdots+\gamma_{d}^{(j)}
$$

as a sum of linearly independent elements in the semigroup $\Gamma_{\theta^{(j-1)}}^{(j)}$, there are exactly $q_{0}$ of them in $M\left(\theta^{(j-1)}\right)$.

ii. $M\left(\theta^{(j-1)}, \Gamma_{\theta^{(j-1)}}^{(j)}\right)=M\left(\theta^{(j-1)}\right)=M\left(\theta^{(j)}\right)$ (cf. Notation 4.9).

Moreover, the sequence of cones $\left(\theta^{(j)}\right)$ stabilizes.

Proof. Notice that the vector $\nu$ is in a subdivision of $\theta^{(j)}$ induced by $\Sigma^{(j+1)}$, so that we have $\theta^{(j+1)} \subseteq \theta^{(j)}$. The sequence $\left(\operatorname{codim}_{N_{\mathbf{R}}} \theta^{(j)}\right)_{j}$ is increasing, thus there exists an integer $0 \leq q_{0} \leq d-1$ such that $\operatorname{codim} \theta^{(j)}=q_{0}$, for $j \gg 0$.

Since $\nu \in \operatorname{int}\left(\theta^{(j)}\right) \cap N$, we have the equalities

$$
\Gamma_{\theta^{(j-1)}}^{(j)} \cap\left(\theta^{(j-1)}\right)^{\perp}=\Gamma_{\theta^{(j-1)}}^{(j)} \cap \nu^{\perp}=M\left(\theta^{(j-1)}, \Gamma_{\theta}^{(j)}\right) .
$$

The lattice $M\left(\theta^{(j-1)}, \Gamma_{\theta^{(j-1)}}^{(j)}\right)$ is a sublattice of finite index $i\left(\theta^{(j-1)}, \Gamma_{\theta^{(j-1)}}^{(j)}\right)$ of $M\left(\theta^{(j-1)}\right)$ (see Notation 4.9 and Lemma 3.9) . By definition $M\left(\theta^{(j-1)}\right)$ is a saturated subsemigroup of $M$, and it is also a rank $q_{0}$ lattice. By (20) we deduce that $M\left(\theta^{(j-1)}\right)=M\left(\theta^{(j)}\right)$ and $M\left(\theta^{(j-1)}, \Gamma_{\theta}^{(j)}\right) \subset M\left(\theta^{(j)}, \Gamma_{\theta}^{(j+1)}\right)$. Then, the sequence of indices $\left(i\left(\theta^{(j-1)}, \Gamma_{\theta^{(j-1)}}^{(j)}\right)\right)_{j}$ stabilizes, hence $M\left(\theta^{(j-1)}, \Gamma_{\theta}^{(j)}\right)=M\left(\theta^{(j)}, \Gamma_{\theta}^{(j+1)}\right)$ for $j \gg 0$. The lattice $M\left(\theta^{(j-1)}, \Gamma_{\theta}^{(j)}\right)$ is a priori a sublattice of finite index of $M\left(\theta^{(j-1)}\right)$.

We deal first with the proof of (i). Up to relabeling the vectors we can assume that those $\gamma_{i}^{(j)}$ appearing in (29), which belong to $M\left(\theta^{(j-1)}\right)$ are $\gamma_{1}^{(j)} \ldots, \gamma_{s}^{(j)}$ for $0 \leq s \leq q_{0}$. By (30) these vectors belong to $M\left(\theta^{(j-1)}, \Gamma_{\theta^{(j-1)}}^{(j)}\right)$. Suppose that $s \neq q_{0}$. Since, the images of the $\gamma_{i}^{(j)}, i=s+1, \ldots, d$, generate a rank $d-q_{0}$ sublattice of $M / M\left(\theta^{(j-1)}\right)$, we get that $d-q_{0}$ of them, say for $i=q_{0}+1, \ldots, d$, are linearly independent modulo 
$M\left(\theta^{(j-1)}\right)$. We can find vectors $\tilde{\gamma}_{s+1}^{(j)}, \ldots, \tilde{\gamma}_{q_{0}}^{(j)} \in \Gamma_{\theta^{(j-1)}}^{(j)} \cap \nu^{\perp}$, such that $\gamma_{1}^{(j)} \wedge \ldots \wedge \gamma_{s}^{(j)} \wedge$ $\tilde{\gamma}_{s+1}^{(j)} \wedge \ldots \wedge \tilde{\gamma}_{q_{0}}^{(j)} \neq 0$. Then the vector

$$
m^{\prime}:=\gamma_{1}^{(j)}+\cdots+\gamma_{s}^{(j)}+\tilde{\gamma}_{s+1}^{(j)}+\cdots+\tilde{\gamma}_{q_{0}}^{(j)}+\gamma_{q_{0}+1}^{(j)}+\cdots+\gamma_{d}^{(j)}
$$

verifies that $m^{\prime} \in \mathcal{J}_{\theta^{(j-1)}}^{(j-1)}$. Since $0=\left\langle\nu, \tilde{\gamma}_{j}^{(j)}\right\rangle\left\langle\left\langle\nu, \gamma_{j}^{(j)}\right\rangle\right.$, for $s+1 \leq j \leq q_{0}$ we would have $\left\langle\nu, m^{\prime}\right\rangle\left\langle\left\langle\nu, m^{(j)}\right\rangle\right.$, a contradiction.

Suppose that (ii) does not hold. Then, since $\Gamma_{\theta^{(j-1)}}^{(j)}$ generates the lattice $M$, there exist $\gamma, \gamma^{\prime} \in \Gamma_{\theta^{(j-1)}}^{(j)}$ such that $\gamma-\gamma^{\prime} \in M\left(\theta^{(j-1)}\right) \backslash M\left(\theta^{(j-1)}, \Gamma_{\theta^{(j-1)}}^{(j)}\right)$. In view of (30) we get $0 \neq\langle\nu, \gamma\rangle=\left\langle\nu, \gamma^{\prime}\right\rangle$. Since $\gamma_{q_{0}+1}^{(j)}, \ldots, \gamma_{d}^{(j)}$ define linearly independent vectors in the lattice $M / M\left(\theta^{(j-1)}\right)$ which is of rank $d-q_{0}$, there exists an integer $q_{0}+1 \leq i_{0} \leq d$ such that $\gamma\left(\right.$ resp. $\left.\gamma^{\prime}\right)$ together with $\gamma_{q_{0}+1}^{(j)}, \ldots, \gamma_{i_{0}-1}^{(j)}, \gamma_{i_{0}+1}^{(j)}, \ldots, \gamma_{d}^{(j)}$ are linearly independent modulo $M\left(\theta^{(j-1)}\right)$. Suppose without loss of generality that $i_{0}=d$. Then the vectors $\tilde{\gamma}:=\gamma+\sum_{i=1}^{d-1} \gamma_{i}^{(j)}$ and $\tilde{\gamma}^{\prime}:=\gamma^{\prime}+\sum_{i=1}^{d-1} \gamma_{i}^{(j)}$ belong to $\mathcal{J}_{\theta^{(j-1)}}^{(j)}$, hence $\tilde{\gamma}-m^{(j)}=\gamma-\gamma_{d}^{(j)}$ and $\tilde{\gamma}^{\prime}-m^{(j)}=\gamma^{\prime}-\gamma_{d}^{(j)}$ are both elements of $\Gamma_{\theta^{(j)}}^{(j+1)}$. Since $\left\langle\nu, \gamma_{d}^{(j)}\right\rangle>0$, it follows that $\tilde{\gamma}-\tilde{\gamma}^{\prime}=\gamma-\gamma^{\prime}$ and $\langle\nu, \tilde{\gamma}\rangle=\left\langle\nu, \tilde{\gamma}^{\prime}\right\rangle\left\langle\langle\nu, \gamma\rangle=\left\langle\nu, \gamma^{\prime}\right\rangle\right.$. By repeating this construction, since $\nu \in \sigma \cap N$, in a finite number of steps we reduce to the case when $\langle\nu, \gamma\rangle=\left\langle\nu, \gamma^{\prime}\right\rangle=0$. As we remarked before this implies that $\gamma-\gamma^{\prime} \in M\left(\theta^{(j-1)}, \Gamma_{\theta^{(j-1)}}^{(j)}\right)$, a contradiction.

By Lemma 12.18 and ii. we can assume that $q_{0}=0$. By (21) the sequence of positive integers $\left(\left\langle\nu, m^{(j)}\right\rangle\right)_{j \geq 1}$ is decreasing, hence it stabilizes. Suppose that the sequence $\left(\theta^{(j)}\right)$ does not stabilize. By Proposition 12.20 this implies that the toric variety $T^{\Gamma_{\theta^{(j-1)}}^{(j)}}$ is not smooth for any $j \geq 1$.

Let us fix an integer $j_{1} \gg 0$. By Proposition 12.12 there exists a smallest integer $j_{2}>$ $j_{1}$ such that $m^{\left(j_{2}\right)} \neq m^{\left(j_{1}\right)}$. We consider a representation of $m^{\left(j_{2}\right)} \in \mathcal{J}_{\theta^{\left(j_{2}-1\right)}}^{\left(j_{2}\right)}$ of the form (29). The vector $m^{\left(j_{1}\right)}-m^{\left(j_{2}\right)}$ belongs to the semigroup $\Gamma_{\theta^{\left(j_{2}\right)}}^{\left(j_{2}+1\right)}$ since $m^{\left(j_{1}\right)} \in \mathcal{J}_{\theta^{\left(j_{2}-1\right)}}^{\left(j_{2}\right)}$ by (20). We obtain that $\left\langle\nu, m^{\left(j_{1}\right)}-m^{\left(j_{2}\right)}\right\rangle=0$, hence $m^{\left(j_{1}\right)}-m^{\left(j_{2}\right)} \in \Gamma_{\theta^{\left(j_{2}\right)}}^{\left(j_{2}+1\right)} \cap \nu^{\perp}$. Since $\nu \in \operatorname{int} \theta^{\left(j_{2}\right)}$ and $\operatorname{dim} \theta^{\left(j_{2}\right)}=d$ we deduce also that $\Gamma_{\theta^{\left(j_{2}\right)}}^{\left(j_{2}+1\right)} \cap \nu^{\perp}=\{0\}$, a contradiction, which ends the proof of Proposition 12.22 .

If $\eta \subset N_{\mathbf{R}}$ is a rational convex polyhedral cone the duality between the lattices $N$ and $M$ induces a duality between the lattices $N_{\eta}$ and $M_{\eta}=M / M(\eta)$ and also a duality between $N(\eta)=N / N_{\eta}$ and $M(\eta)=M \cap \eta^{\perp}$ (cf. Notations 4.9).

If $0 \neq \eta$ is a stable cone one can consider for $j \gg 0$ the orbit closure $T_{\Sigma^{(j-1)}}^{\Gamma^{(j)}}(\eta)$ associated to $\eta \in \Sigma^{(j-1)}$. By convenience we recall the notations to describe this toric variety in this case. See Lemma 4.10 and Notations 4.9.

Notation 12.23. If $\eta \in \Sigma^{(j-1)}$ is a stable cone the variety $T_{\Sigma^{(j-1)}}^{\Gamma^{(j)}}(\eta)$ is covered by charts defined by the semigroups $\Gamma_{\theta^{(j-1)}}^{(j)} \cap \eta^{\perp}$, for $\eta \leq \theta^{(j-1)}$ and $\theta^{(j-1)} \in \Sigma^{(j-1)}$. Notice that the semigroup $\Gamma_{\theta^{(j-1)}}^{(j)} \cap \eta^{\perp}$ is a face of $\Gamma_{\theta^{(j-1)}}^{(j)}$, and it spans the lattice $M\left(\eta, \Gamma_{\eta}^{(j)}\right)=\Gamma_{\eta}^{(j)} \cap \eta^{\perp}$ by Lemma 3.9. By Proposition 12.22 ii. this lattice is equal to $M(\eta)$ if $j \gg 0$. The fan $\Sigma^{(j-1)}(\eta)$ consists of the images $\theta^{(j-1)}(\eta)$ of cones $\theta^{(j-1)} \in \Sigma^{(j-1)}$ in $N(\eta)_{\mathbf{R}}$. The cone $\theta^{(j-1)}(\eta)$ is the dual cone of $\mathbf{R}_{\geq 0}\left(\Gamma_{\theta^{(j-1)}}^{(j)} \cap \eta^{\perp}\right)$. We denote by $\mathcal{J}_{\theta^{(j-1)}}^{(j)}(\eta)$ the logarithmic jacobian ideal of $k\left[t^{\Gamma_{\theta(j-1)}^{(j)} \cap \eta^{\perp}}\right]$.

The following technical lemma will be useful. 
Lemma 12.24. Let $0 \neq \eta$ be a stable cone of codimension $d_{0}<d$. We denote by

$$
\pi: M_{\mathbf{R}} \rightarrow M_{\mathbf{R}} / M(\eta)_{\mathbf{R}}, \quad \alpha \mapsto \tilde{\alpha}
$$

the canonical projection. If $\left(\theta^{(j)}\right)_{j}$ is a sequence such that

$$
\theta^{(j)} \in \Sigma^{(j)}, \theta^{(j)} \supset \theta^{(j+1)} \text { and } \eta \leq \theta^{(j)},
$$

then for $j \gg 0$ we have:

i. $\pi\left(\Gamma_{\theta^{(j-1)}}^{(j)}\right)=\pi\left(\Gamma_{\theta^{(j)}}^{(j+1)}\right)$ and the semigroup $\tilde{\Gamma}_{\eta}:=\pi\left(\Gamma_{\theta^{(j-1)}}^{(j)}\right)$ is generated by a basis $\tilde{e}_{d_{0}+1}, \ldots, \tilde{e}_{d}$ of $M / M(\eta)$.

ii. If $m^{(j)}$ is the vertex of the polyhedron $\mathcal{N}\left(\mathcal{J}_{\theta^{(j-1)}}^{(j)}\right)$ such that

$$
\operatorname{ord}_{\mathcal{J}^{(j)}}(\nu)=\left\langle\nu, m^{(j)}\right\rangle, \quad \forall \nu \in \theta^{(j)},
$$

then for any representation of the form (29) of $m^{(j)}$ as a sum of linearly independent vectors in $\Gamma_{\theta^{(j-1)}}^{(j)}$ then exactly $d_{0}$ of the $\gamma_{i}^{(j)}$ belong to $\Gamma_{\theta^{(j-1)}}^{(j)} \cap \eta^{\perp}$, say for $i=1, \ldots, d_{0}$, while $\left\langle\nu_{\eta}, \gamma_{i}^{(j)}\right\rangle=1$ for $i=d_{0}+1, \ldots, d$.

iii. The vector $m^{(j)}$ belongs to the face $\mathcal{F}_{\theta^{(j-1)}}^{(j)}$ of $\mathcal{N}\left(\mathcal{J}_{\theta^{(j-1)}}^{(j)}\right)$ determined by $\nu_{\eta}$ (cf. Definition 12.17). The face $\mathcal{F}_{\theta^{(j-1)}}^{(j)}$ is the Minkowski sum

$$
\mathcal{N}\left(\mathcal{J}_{\theta^{(j-1)}}^{(j)}(\eta)\right)+\mathcal{P}_{\theta^{(j-1)}}^{(j)},
$$

where $\mathcal{P}_{\theta^{(j-1)}}^{(j)}$ is the convex hull of the set $\bigcup \delta_{i_{d_{0}+1}}^{(j)}+\cdots+\delta_{i_{d}}^{(j)}+\left(\check{\theta}^{(j-1)} \cap \eta^{\perp}\right)$, for $\delta_{i_{d_{0}+1}}^{(j)}, \ldots, \delta_{i_{d}}^{(j)} \in \Gamma_{\theta^{(j-1)}}^{(j)}$ such that $\delta_{i_{d_{0}+1}}^{(j)} \wedge \cdots \wedge \delta_{i_{d}}^{(j)} \neq 0$, and $\left\langle\nu_{\eta}, \delta_{i_{l}}^{(j)}\right\rangle=1$ for $l=d_{0}+1, \ldots, d$.

Proof. Since $\eta$ is a stable cone we get that $M(\eta)=M\left(\eta, \Gamma_{\eta}^{(j)}\right)$, by applying Proposition 12.22 to the constant sequence of cones $\eta_{j}:=\eta$. By Lemma 12.18 the cone $\eta \subset\left(N_{\eta}\right)_{\mathbf{R}}$ is a stable cone for the semigroup $\pi\left(\Gamma_{\eta}^{(j)}\right)$ for $j \geq j_{0} \gg 0$. By Proposition 12.20 the sequence of semigroups $\left(\pi\left(\Gamma_{\eta}^{(j)}\right)\right)_{j \geq j_{0}}$ stabilizes and $\pi\left(\Gamma_{\eta}^{(j)}\right)$ is generated by a basis of $M / M(\eta)$, for $j \gg 0$. By Lemma 3.9 we have that $\Gamma_{\eta}^{(j)}=\Gamma_{\theta^{(j-1)}}^{(j)}+\mathbf{Z}_{\geq 0}\left(-u_{j}\right)$, for any $u_{j} \in \Gamma_{\theta^{(j-1)}}^{(j)}$ which belongs to $\operatorname{int}\left(\check{\theta}^{(j-1)} \cap \eta^{\perp}\right)$. We deduce that $\pi\left(\Gamma_{\theta^{(j-1)}}^{(j)}\right)=\pi\left(\Gamma_{\eta}^{(j)}\right)$ for $j \gg 0$.

Since $\eta \leq \theta^{(j)}$ we get from (32) that $\operatorname{ord}_{\mathcal{J}^{(j)}}\left(\nu_{\eta}\right)=\left\langle\nu_{\eta}, m^{(j)}\right\rangle$. This implies that $m^{(j)}$ belongs to $\mathcal{F}_{\theta^{(j-1)}}^{(j)}$. Notice also that $m^{(j)}$ belongs to $\mathcal{J}_{\eta}^{(j)}$. By Proposition $12.22 \mathrm{i}$., for any representation of $m^{(j)}$ of the form (29),$d_{0}$ of the $\gamma_{i}^{(j)}$ belong to $\eta^{\perp}$, say for $i=1, \ldots, d_{0}$. By Lemma 11.6 the vectors $\pi\left(\gamma_{i}^{(j)}\right)=\tilde{\gamma}^{(j)}, i=d_{0}+1, \ldots, d$ are linearly independent elements of $\Gamma_{\eta}$, such that $\tilde{m}^{(j)}=\sum_{i} \tilde{\gamma}_{i}^{(j)}$ belongs to the logarithmic jacobian ideal $\tilde{\mathcal{J}}_{\eta}$ of $k\left[t^{\tilde{\Gamma}_{\eta}}\right]$. We know that $\langle\nu, m\rangle=\langle\nu, \tilde{m}\rangle$ for any $m \in M$ and $\nu \in \eta$, thus the vector $\tilde{m}^{(j)}$ is in the face of $\mathcal{N}\left(\tilde{\mathcal{J}}_{\eta}\right)$ determined by $\nu_{\eta}$. By i. the semigroup $\tilde{\Gamma}_{\eta}$ is regular, hence we deduce that $\tilde{m}^{(j)}=\sum_{i=d_{0}+1}^{d} \tilde{e}_{i}$ and, up to relabeling, $\tilde{e}_{i}=\tilde{\gamma}_{i}^{(j)}, i=d_{0}+1, \ldots, d$. This ends the proof of ii. and also shows that $m^{(j)} \in \mathcal{N}\left(\mathcal{J}_{\theta^{(j-1)}}^{(j)}(\eta)\right)+\mathcal{P}_{\theta^{(j-1)}}^{(j)}$.

We have also shown the equalities

$$
\operatorname{ord}_{\mathcal{J}^{(j)}}\left(\nu_{\eta}\right)=\left\langle\nu_{\eta}, m^{(j)}\right\rangle=\left\langle\nu_{\eta}, \tilde{m}^{(j)}\right\rangle=d-d_{0} .
$$

Finally, we remark that the argument given above applies more generally for any vector $m \in \mathcal{J}_{\theta^{(j-1)}}^{(j)}$ in the face $\mathcal{F}_{\theta^{(j-1)}}^{(j)}$. This implies that iii. holds. 
Proposition 12.25. If $0 \neq \eta$ is a stable cone and if $\left(\theta^{(j)}\right)_{j}$ is a sequence of cones of the form (31) such that $\theta^{(j)}$ contains $\eta$ as a face of codimension one, then

$$
M\left(\theta^{(j-1)}, \Gamma_{\theta^{(j-1)}}^{(j)}\right)=M\left(\theta^{(j-1)}\right) \text { for } j \gg 0,
$$

and the sequence of cones $\left(\theta^{(j)}\right)_{j \geq I}$ stabilizes.

Proof. We denote by $q_{0}$ the integer $\operatorname{codim}_{N_{\mathbf{R}}} \theta^{(j)}$ for $j \gg 0$ and by $d_{0}$ the codimension of $\eta$. Notice that $0 \leq q_{0}<d-1$ since $0 \neq \eta \leq \theta^{(j)}$.

We deal first with the proof of (33). By the argument given in the proof of Proposition 12.22 we get $M\left(\theta^{(j-1)}\right)=M\left(\theta^{(j)}\right)$ and $M\left(\theta^{(j-1)}, \Gamma_{\theta^{(j-1)}}^{(j)}\right)=M\left(\theta^{(j)}, \Gamma_{\theta^{(j)}}^{(j+1)}\right)$ for $j \gg 0$. We recall that for $j \gg 0$ the lattice spanned by the face $\Gamma_{\theta^{(j-1)}}^{(j)} \cap \eta^{\perp}$ is equal to $M(\eta)$ (see Notations 12.23).

If (33) were not true then there exists $\gamma, \gamma^{\prime} \in \Gamma_{\theta^{(j-1)}}^{(j)}$ such that $\gamma-\gamma^{\prime} \in M\left(\theta^{(j-1)}\right) \backslash$ $M\left(\theta^{(j-1)}, \Gamma_{\theta^{(j-1)}}^{(j)}\right)$. Notice then that $\left\langle\nu_{\eta}, \gamma\right\rangle=\left\langle\nu_{\eta}, \gamma^{\prime}\right\rangle$ since $M\left(\theta^{(j-1)}\right) \subset M(\eta)$ by duality.

By Lemma 12.24, if $m^{(j)} \in \mathcal{J}_{\theta^{(j-1)}}^{(j)}$ is such that $\operatorname{ord}_{\mathcal{J}^{(j)}}(\nu)=\left\langle\nu, m^{(j)}\right\rangle$ for any $\nu \in \theta^{(j)}$ then for any representation of $m^{(j)}$ of the form (29),$d_{0}$ of the $\gamma_{i}^{(j)}$ belong to $M(\eta)$.

By applying the argument of Proposition 12.22 we can assume, replacing $j$ by a bigger number, that $\left\langle\nu_{\eta}, \gamma\right\rangle=0$, that is $\gamma$ and $\gamma^{\prime}$ belong to the face $\Gamma_{\theta^{(j-1)}}^{(j)} \cap \eta^{\perp}$.

By hypothesis $\eta \leq \theta^{(j-1)}$ is a face of codimension one. The image of $\check{\theta}^{(j-1)} \cap \eta^{\perp}$ in $M(\eta)_{\mathbf{R}} / M\left(\theta^{(j-1)}\right)_{\mathbf{R}}$ is the dual cone of the image $\bar{\theta}^{(j-1)}$ of $\theta^{(j-1)}$ in $\left(N_{\theta^{(j-1)}}\right)_{\mathbf{R}} /\left(N_{\eta}\right)_{\mathbf{R}}$. Notice that the lattice $N_{\theta^{(j-1)}} / N_{\eta}$ and the cone $\bar{\theta}^{(j-1)}$ are independent of $j$ for $j \gg 0$. We denote by $\bar{\nu}$ the generator of the semigroup $\bar{\theta}^{(j-1)} \cap\left(N_{\theta^{(j-1)}} / N_{\eta}\right)$ and by $\bar{\alpha}$ the class of $\alpha \in M(\eta)$ modulo $M\left(\theta^{(j-1)}\right)$.

Among those $\gamma_{i}^{(j)}$ which belong to $\eta^{\perp}$ there exists at least one, say $\gamma_{d}^{(j)}$, which does not belong to $M\left(\theta^{(j-1)}\right)$, hence $\left\langle\bar{\nu}, \bar{\gamma}_{d}^{(j)}\right\rangle \neq 0$. Notice also that $0 \neq\langle\bar{\nu}, \bar{\gamma}\rangle=\left\langle\bar{\nu}, \bar{\gamma}^{\prime}\right\rangle$. Then, we apply the same argument as in the proof of Proposition $12.22 \mathrm{i}$. to get that $\gamma-\gamma_{d}^{(j)}$ and $\gamma^{\prime}-\gamma_{d}^{(j)}$ belong to $\Gamma_{\theta^{(j)}}^{(j+1)} \cap \eta^{\perp}$ and $\left\langle\bar{\nu}, \bar{\gamma}-\bar{\gamma}_{d}^{(j)}\right\rangle\langle\langle\bar{\nu}, \bar{\gamma}\rangle$. By iterating this procedure, replacing $\gamma$ and $\gamma^{\prime}$ by $\gamma-\gamma_{d}^{(j)}$ and $\gamma-\gamma_{d}^{(j)}$, respectively, we reduce to the case $0=\langle\bar{\nu}, \bar{\gamma}\rangle=\left\langle\bar{\nu}, \bar{\gamma}^{\prime}\right\rangle$. But this implies that $\gamma, \gamma^{\prime}$ belong to $M\left(\theta^{(j-1)}, \Gamma_{\theta^{(j-1)}}^{(j)}\right)$, contradicting the hypothesis. This ends the proof of the equality (33).

We prove now that the sequence $\left(\theta^{(j)}\right)$ stabilizes. Since (33) holds, by Lemma 12.18 we can assume that $q_{0}=0$. Then, by hypothesis the lattice $M(\eta)$ is of rank one. The cone $\check{\theta}^{(j-1)} \cap \eta^{\perp} \subset M(\eta)_{\mathbf{R}}$ is a one dimensional face of $\check{\theta}^{(j-1)}$ for all $j \gg 0$. Since $\check{\theta}^{(j-1)} \subset \check{\theta}^{(j)}$ we get that the cone $\check{\vartheta}:=\check{\theta}^{(j-1)} \cap \eta^{\perp}$ is independent of $j$, for $j \gg 0$.

With notations of Lemma 12.24, the Minkowski sum $\pi^{-1}\left(\tilde{e}_{i}\right) \cap \Gamma_{\theta^{(j-1)}}^{(j)}+\check{\vartheta}$ is an affine one dimensional cone with only one vertex $\gamma_{i}^{(j)}$, which belongs to $\Gamma_{\theta^{(j-1)}}^{(j)}$, for $i=2, \ldots, d$. We denote by $\gamma_{1}^{(j)}$ the smallest generator of the rank one semigroup $\Gamma_{\theta^{(j-1)}}^{(j)} \cap \check{\vartheta}$. Notice that $\gamma_{1}^{(j)}$ is independent of $j$ for $j \gg 0$.

By Lemma 12.24 the vector $m^{(j)}=\gamma_{1}^{(j)}+\cdots+\gamma_{d}^{(j)} \in \mathcal{J}_{\theta^{(j-1)}}^{(j)}$ is the unique vertex of the face $\mathcal{F}_{\theta^{(j-1)}}^{(j)}=m^{(j)}+\check{\vartheta}$. Since $\nu_{\eta} \in \theta^{(j)}$ the cone $\theta^{(j)}$ is dual to the cone spanned by $\left\{\gamma-m^{(j)} \mid \gamma \in \mathcal{J}_{\theta^{(j-1)}}^{(j)}\right\}$.

Claim 12.26. The semigroup $\Gamma_{\theta^{(j-1)}}^{(j)} \cap \eta^{\perp}$ is regular for $j \gg 0$. 
Proof of the claim. By Proposition 12.22 the semigroup $\Gamma_{\theta^{(j-1)}}^{(j)} \cap \eta^{\perp}=\Gamma_{\theta^{(j-1)}}^{(j)} \cap \check{\vartheta}$ generates the group $M(\eta)$, for $j \gg 0$. If it has only one generator the result follows directly. Assume that it has at least two generators. Let us denote by $\delta>\gamma_{1}^{(j)}$ the second element by order of size. The element $n^{(j)}=\gamma_{2}^{(j)}+\cdots+\gamma_{1}^{(j)}+\delta$ belongs to $\mathcal{J}_{\theta^{(j-1)}}^{(j)}$ so that $n^{(j)}-m^{(j)}=\delta-\gamma_{1}^{(j)}$ is in $\Gamma_{\theta^{(j)}}^{(j+1)}$. We see that after finitely many steps the smallest generator of our semigroup has decreased, so in the end we reach the generator of the regular semigroup $\check{\vartheta} \cap M$, which proves the result. This is similar to the resolution process for one dimensional affine toric varieties by Semple-Nash modifications.

By Lemma 12.24 and Claim 12.26 the elements $\gamma_{1}^{(j)}, \ldots, \gamma_{d}^{(j)}$ form a basis of $M$.

The expansion of $\gamma \in \Gamma_{\theta^{(j-1)}}^{(j)}$ in terms of the basis $\gamma_{1}^{(j)}, \ldots, \gamma_{d}^{(j)}$ is of the form

$$
\gamma=a_{1} \gamma_{1}^{(j)}+\cdots+a_{d} \gamma_{d}^{(j)}
$$

where $a_{i} \in \mathbf{Z}_{\geq 0}$ and $a_{d} \in \mathbf{Z}$. If $\gamma$ is of the form (34) then we have $\left\langle\nu_{\eta}, \gamma\right\rangle=\sum_{i=2}^{d} a_{i}$. In particular, we get that $\operatorname{ord}_{\mathcal{J}^{(j)}}\left(\nu_{\eta}\right)=\left\langle\nu_{\eta}, m^{(j)}\right\rangle=d-1$.

We denote by $G^{(j)}$ the minimal generating system of the semigroup $\Gamma_{\theta^{(j-1)}}^{(j)}$, and by $g^{(j)}$ the maximum of $\nu_{\eta}$ on the set $G^{(j)}$.

Notice that the elements $\gamma_{1}^{(j)}, \ldots, \gamma_{d}^{(j)}$ belong to $G^{(j)}$ by our assumptions. If a generator $\gamma \in \Gamma^{(j)}$ is different from $\gamma_{1}^{(j)}, \ldots, \gamma_{d}^{(j)}$ then the coefficient $a_{1}$ in (34) is $<0$, and $\left\langle\nu_{\eta}, \gamma\right\rangle \geq 2$. Otherwise $\gamma$ would be in the semigroup generated by the $\gamma_{i}^{(j)}$, contradicting the minimality of the generating system $G^{(j)}$. We deduce that the equality $g^{(j)}=1$ implies that $G^{(j)}=\left\{\gamma_{i}^{(j)}\right\}_{i=1}^{d}$, hence in this case the semigroup $\Gamma_{\theta^{(j-1)}}^{(j)}$ is regular.

Claim 12.27. If $g^{(j)}>1$ there exists an integer $t_{0} \geq 1$ such that $g^{\left(j+t_{0}\right)}<g^{(j)}$.

Proof of the claim. By Lemma 12.1 the semigroup $\Gamma_{\theta^{(j)}}^{(j+1)}$ is generated by $\gamma_{1}^{(j)}, \ldots, \gamma_{d}^{(j)}$ and vectors of the form $\gamma-\gamma_{l}^{(j)}$, where $\gamma \in G^{(j)}, \gamma \neq \gamma_{l}^{(j)}$ and the $l$-th coordinate of $\gamma$ in the basis $\gamma_{1}^{(j)}, \ldots, \gamma_{d}^{(j)}$ is non-zero (in particular $\left\langle\nu_{\eta}, \gamma\right\rangle \geq 2$ ). We say that vectors of this form are followers of $\gamma$. Notice that $\gamma-\gamma_{1}^{(j)}$ is always a follower of $\gamma$ with $\left\langle\nu_{\eta}, \gamma\right\rangle=\left\langle\nu_{\eta}, \gamma-\gamma_{1}^{(j)}\right\rangle$, while $\left\langle\nu_{\eta}, \gamma-\gamma_{l}^{(j)}\right\rangle\left\langle\left\langle\nu_{\eta}, \gamma\right\rangle\right.$ if $l \neq 1$.

We deduce also that $m^{(j)} \neq m^{(j+1)}$ if and only if there is an element $\gamma \in G^{(j)}$ with $\left\langle\nu_{\eta}, \gamma\right\rangle=2$. Indeed, if $\left\langle\nu_{\eta}, \gamma\right\rangle=2$ then there is $2 \leq i \leq d$ such that $\gamma-\gamma_{i}^{(j-1)}$ is a follower of $\gamma$ with $\left\langle\nu_{\eta}, \gamma-\gamma_{i}^{(j-1)}\right\rangle=1$. This implies that $\gamma_{i}^{(j)}$ is in the semigroup generated by $\gamma-\gamma_{i}^{(j)}$ and $\gamma_{d}^{(j)}$, thus $\gamma_{i}^{(j)}$ does not belong to $G^{(j+1)}$ hence $\gamma_{i}^{(j)} \neq \gamma_{i}^{(j+1)}$ and $m^{(j)} \neq m^{(j+1)}$. The converse is deduced similarly.

Assume that $m^{(j)}=m^{(j+1)}=\cdots=m^{\left(j+t_{0}-1\right)}$ for some $t_{0} \geq 1$. Let $\gamma \in G^{(j)}$ be such that $\left\langle\nu_{\eta}, \gamma\right\rangle=g^{(j)}$. If $\gamma$ is of the form (34) the followers of $\gamma$ after at most $t_{0}$ iterations are $\gamma_{\alpha}=\sum_{i=1}^{d}\left(a_{i}-\alpha_{i}\right) \gamma_{i}^{(j)}$, where $\alpha=\left(\alpha_{1}, \ldots, \alpha_{d}\right) \in \mathbf{Z}_{\geq 0}^{d}$ verify that $\sum_{i=1}^{d} \alpha_{i} \leq t_{0}, \alpha_{i} \leq a_{i}$ for $i=2, \ldots, d$ and $\left\langle\nu_{\eta}, \gamma_{\alpha}\right\rangle=\sum_{i=2}^{d}\left(a_{i}-\alpha_{i}\right) \geq 1$. Those $\gamma_{\alpha}$ with $\left\langle\nu_{\eta}, \gamma_{\alpha}\right\rangle=\left\langle\nu_{\eta}, \gamma\right\rangle$ are precisely $\gamma-l \gamma_{1}^{(j)}$ for $0 \leq l \leq t_{0}$. The elements $\gamma-l \gamma_{1}^{(j)}, l=0, \ldots, t_{0}-1$ do not belong to $G^{\left(j+t_{0}\right)}$ since they are in the semigroup generated by $\gamma_{1}^{(j)}$ and $\gamma-t_{0} \gamma_{1}^{(j)}$.

Assume that $t_{0}>1$ is the smallest integer such that $m^{(j)} \neq m^{\left(j+t_{0}\right)}$. Notice that $t_{0} \leq g^{(j)}-1$, otherwise we would get a follower $\gamma_{\alpha}$ of $\gamma$ with $\left\langle\nu_{\eta}, \gamma_{\alpha}\right\rangle=1$, which is necessarily different from the $\gamma_{i}^{(j)}, i=2, \ldots, d$, and then $m^{(j)} \neq m^{\left(j+g^{(j)}-1\right)}$, a contradiction. 
There exists $0 \neq\left(p_{2}, \ldots, p_{d}\right) \in \mathbf{Z}_{\geq 0}^{d-1}$ such that

$$
\gamma_{i}^{\left(j+t_{0}\right)}=\gamma_{i}^{(j)}-p_{i} \gamma_{1}^{(j)}, i=2, \ldots, d, \text { and } \gamma_{1}^{\left(j+t_{0}\right)}=\gamma_{1}^{(j)} \text {. }
$$

We deduce the expansion

$$
\gamma_{\alpha}=\sum_{i=2}^{d}\left(a_{i}-\alpha_{i}\right) \gamma_{i}^{\left(j+t_{0}\right)}+\left(a_{1}-\alpha_{1}+\sum_{i=2}^{d} p_{i}\left(a_{i}-\alpha_{i}\right)\right) \gamma_{1}^{\left(j+t_{0}\right)} .
$$

If $a_{i} p_{i}=0$ for $i=2, \ldots, d$ we iterate this procedure replacing $\gamma$ by $\gamma-t_{0} \gamma_{1}^{(j)}$. In at most $\left\langle\nu_{\eta}, \gamma\right\rangle-1$ steps we get to the situation where at least one of the $a_{i} p_{i}$ is non-zero, say $a_{d} p_{d} \neq 0$ for simplicity. We prove that $\alpha_{0}=\left(t_{0}-1,0, \ldots, 0,1\right)$ defines a follower of $\gamma$ such that $\gamma-t_{0} \gamma_{1}^{(j)}$ belongs to the semigroup generated by $\gamma_{\alpha_{0}}$ and $\gamma_{i}^{\left(j+t_{0}\right)}, i=1, \ldots, d$, in particular $\gamma-t_{0} \gamma_{1}^{(j)}$ does not belong to $G^{\left(j+t_{0}\right)}$. We check this assertion by verifying that the coefficient of the term $\gamma_{i}^{\left(j+t_{0}\right)}$ in the expansion (35) of $\gamma_{\alpha_{0}}$ is less than or equal to the corresponding coefficient in the expansion (35) of $\gamma-t_{0} \gamma_{1}^{(j)}$ for $i=1, \ldots, d$. If $i=d$ we get a strict inequality. The inequality is trivial for $i=2, \ldots, d-1$. If $i=1$ we have to show the inequality:

$$
a_{1}-\left(t_{0}-1\right)-p_{d}+\sum_{i=2}^{d} p_{i} a_{i} \leq a_{1}-t_{0}+\sum_{i=2}^{d} p_{i} a_{i} .
$$

This inequality is equivalent to $p_{d} \geq 1$, which holds since $a_{d} p_{d} \neq 0$.

Since there is a finite number of $\gamma \in G^{(j)}$ with $\left\langle\nu_{\eta}, \gamma\right\rangle=g^{(j)}$ there exists an integer $t_{2} \geq 0$ such that $g^{\left(j+t_{2}\right)}<g^{(j)}$ as claimed.

Using this claim and induction there exists an integer $t_{1} \geq 1$ such that $g^{\left(j+t_{1}\right)}=1$, hence the semigroup $\Gamma_{\theta^{(j-1)}}^{(j)}$ is regular. This ends the proof of Proposition 12.25.

Definition 12.28. The nested sequence of cones $\left(\theta^{(j)}\right)_{j}$ is distinguished if there exist a stable cone $0 \neq \eta$, an integer $I$, and a sequence of faces of $\theta^{(j)}$ for $j \geq I$,

$$
\eta=\zeta_{0}^{(j)} \leq \zeta_{1}^{(j)} \leq \cdots \leq \zeta_{l_{0}}^{(j)}=\theta^{(j)},
$$

such that $\operatorname{dim} \zeta_{i}^{(j)}=\operatorname{dim} \eta+i$ and $\zeta_{i}^{(j)} \supset \zeta_{i}^{(j+1)}$ for $i=0, \ldots, l_{0}$ and $l_{0} \leq \operatorname{codim} \eta$.

Proposition 12.29. If the sequence $\left(\theta^{(j)}\right)_{j}$ is distinguished then it stabilizes.

Proof. Let $\left(\theta^{(j)}\right)_{j}$ be a distinguished sequence as above. We consider the sequence of faces $\left(\zeta_{1}^{(j)}\right)_{j}$. Since $\eta$ is a face of codimension one of $\zeta_{1}^{(j)}$ we get that the sequence $\left(\zeta_{1}^{(j)}\right)_{j}$ stabilizes by Proposition 12.25. We proceed replacing $\eta=\zeta_{0}^{(j)}$ and $\zeta_{1}^{(j)}$ by $\zeta_{1}^{(j)}$ and $\zeta_{2}^{(j)}$ respectively, in the previous argument, and then the result follows by induction on the length $l_{0}$ of the sequence (36) .

Remark 12.30. If $0 \neq \eta$ is a stable cone, for $j \gg 0$, the blowing up of the logarithmic jacobian ideal induces a proper toric modification $\xi_{\eta}^{(j)}: T_{\Sigma^{(j+1)}}^{\Gamma^{(j+1)}}(\eta) \rightarrow T_{\Sigma^{(j)}}^{\Gamma^{(j)}}(\eta)$. As we explain below, the $\operatorname{map} \xi_{\eta}^{(j)}$ is not necessarily equal to the blowing up of the logarithmic jacobian ideal of $T_{\Sigma^{(j)}}^{\Gamma^{(j)}}(\eta)$.

Proposition 12.31. Given a stable cone $\eta$ of codimension $d_{0}$, a vector $0 \neq w \in N(\eta)$ and a sequence of cones $\theta^{(j)}(\eta) \in \Sigma^{(j)}(\eta)$ such that $\theta^{(j)}(\eta) \supset \theta^{(j+1)}(\eta)$ for $j \gg 0$. Then, if $w \in \operatorname{int}\left(\theta^{(j)}(\eta)\right)$ for $j \gg 0$, we have that

$$
M\left(\theta^{(j-1)}(\eta), \Gamma_{\theta^{(j-1)}}^{(j)} \cap \eta^{\perp}\right)=M\left(\theta^{(j-1)}(\eta)\right) \text { for } j \gg 0,
$$

and the sequence $\theta^{(j)}(\eta)$ stabilizes. 
Proof. We use notations 12.23. We denote by $q_{0}$ the codimension of $\theta^{(j)}(\eta)$ for $j \gg 0$. If $\theta^{(j-1)} \in \Sigma^{(j-1)}$ is a cone such that $\eta \leq \theta^{(j-1)}$ and its image in $N(\eta)$ is equal to $\theta^{(j-1)}(\eta)$ then by definition we get $M\left(\theta^{(j-1)}(\eta), \Gamma_{\theta^{(j-1)}}^{(j)} \cap \eta^{\perp}\right)=M\left(\theta^{(j-1)}, \Gamma_{\theta^{(j-1)}}^{(j)} \cap \theta^{(j-1) \perp}\right) \subset$ $M\left(\eta, \Gamma_{\eta}^{(j)}\right)$ and $M\left(\theta^{(j-1)}(\eta)\right)=M\left(\theta^{(j-1)}\right) \subset M(\eta)$.

We get that $M\left(\theta^{(j-1)}(\eta), \Gamma_{\theta^{(j-1)}}^{(j)} \cap \eta^{\perp}\right)=M\left(\theta^{(j)}(\eta), \Gamma_{\theta^{(j)}}^{(j+1)} \cap \eta^{\perp}\right)$ and $M\left(\theta^{(j-1)}(\eta)\right)=$ $M\left(\theta^{(j)}(\eta)\right)$ for $j \gg 0$. Then, the proof of formula (37) follows similarly as that of (33) and of Proposition 12.22 ii.

We deduce from (37) and Lemma 12.24 that it is enough to prove the result in the case $\operatorname{codim} \theta^{(j)}(\eta)=0$. In this case, the cone $\theta^{(j-1)}(\eta)$ is the image of a cone $\theta^{(j-1)} \in \Sigma^{(j)}(d)$ by the projection $\pi: N_{\mathbf{R}} \rightarrow N(\eta)_{\mathbf{R}}$.

The charts of the blowing up of the logarithmic jacobian ideal of $T_{\theta^{(j-1)}}^{\Gamma^{(j)}}$ which are defined by those $d$ dimensional cones which contain $\eta$, are in bijection with the vertices of the face $\mathcal{F}_{\theta^{(j-1)}}^{(j)}$ of the Newton polyhedron of the logarithmic jacobian ideal $\mathcal{J}_{\theta^{(j-1)}}^{(j)}$ determined by the vector $\nu_{\eta}$. One of these charts $T_{\theta^{(j)}}^{\Gamma^{(j+1)}}$, corresponding to a vertex $m^{(j)}$ of $\mathcal{F}_{\theta^{(j-1)}}^{(j)}$ is such that the image of the cone $\theta^{(j)}$ in $N(\eta)$ is equal to the cone $\theta^{(j)}(\eta)$ in the sequence.

By Lemma 12.24 we can choose a representation $m^{(j)}=\gamma_{1}^{(j)}+\cdots+\gamma_{d}^{(j)}$ as sum of linearly independent elements of $\Gamma_{\theta^{(j-1)}}^{(j)}$, such that

$$
\left\langle\nu_{\eta}, \gamma_{i}^{(j)}\right\rangle=\left\{\begin{array}{lll}
0 & \text { if } \quad 1 \leq i \leq d_{0} \\
1 & \text { if } \quad d_{0}+1 \leq i \leq d .
\end{array}\right.
$$

Then we have that $m^{(j)}=\bar{m}^{(j)}+m^{\prime(j)}$ where

$$
\bar{m}^{(j)}=\gamma_{1}^{(j)}+\cdots+\gamma_{d_{0}}^{(j)} \text { and } m^{\prime(j)}=\gamma_{d_{0}+1}^{(j)}+\cdots+\gamma_{d}^{(j)} .
$$

By hypothesis $w \in N(\eta)$ is in the relative interior of $\theta^{(j)}(\eta)$. We get that $\bar{m}^{(j)}$ is the vertex of the Newton polyhedron of the logarithmic jacobian ideal $\mathcal{J}_{\theta^{(j-1)}}^{(j)}(\eta)$ of $k\left[t^{\Gamma_{\theta^{(j-1)}}^{(j)}}\right]$ determined by $w$ (see Lemma 12.24).

Remark that the chart associated to $\bar{m}^{(j)}$ of the blowing up of the logarithmic jacobian ideal $\mathcal{J}_{\theta^{(j-1)}}^{(j)}(\eta)$ of $k\left[t_{\theta^{(j-1)}}^{\Gamma^{(j)}}\right]$ is defined by the semigroup $S^{(j+1)}$ generated by $\Gamma_{\theta^{(j-1)}}^{(j)} \cap \eta^{\perp}$ and vectors of the form $\gamma-\bar{m}^{(j)}$ for $\gamma$ in $\mathcal{J}_{\theta^{(j-1)}}^{(j)}(\eta)$. The inclusion $S^{(j+1)} \subset \Gamma_{\theta^{(j)}}^{(j+1)} \cap \eta^{\perp}$, may be strict (cf. Remark 12.30). The reason is the following. If $\gamma \in \Gamma_{\theta^{(j-1)}}^{(j)}$ and if $\gamma \wedge \gamma_{1}^{(j)} \wedge \cdots \wedge \gamma_{i-1}^{(j)} \wedge \gamma_{i+1}^{(j)} \wedge \cdots \wedge \gamma_{d}^{(j)} \neq 0$ then $\delta:=\gamma+m^{(j)}-\gamma_{i}^{(j)}$ belongs to $\mathcal{J}_{\theta^{(j-1)}}^{(j)}$ and $\delta-m^{(j)}=\gamma-\gamma_{i}^{(j)} \in \Gamma_{\theta(j)}^{(j+1)}$. If in addition, $\left\langle\nu_{\eta}, \gamma\right\rangle=\left\langle\nu_{\eta}, \gamma_{i}^{(j)}\right\rangle$ we obtain that $\gamma-\gamma_{i}^{(j)}$ belongs to $\Gamma_{\theta^{(j)}}^{(j+1)} \cap \eta^{\perp}$, even if $\gamma \notin \eta^{\perp}$.

Notice that the sequence $\left(\left\langle w, \bar{m}^{(j)}\right\rangle\right)$ is stationary since $w$ belongs to the interior of $\theta^{(j-1)}(\eta)$. There exists $j_{1} \geq 0$, such that the sequence $\left(\left\langle w, \bar{m}^{(j)}\right\rangle\right)$ is constant for $j \geq j_{1}$.

If the sequence $\left(\theta^{(j)}(\eta)\right)$ does not stabilize then the toric variety $T^{\Gamma^{(j)}}{ }^{(j-1)}$ is not smooth for any integer $j$. By Proposition 12.12, if the toric variety $T^{\Gamma_{\theta^{\left(j_{1}-1\right)}}^{\left(j_{1}\right)}}$ is not smooth then there is a smallest $j_{2}>j_{1}$ such that $m^{\left(j_{1}\right)} \neq m^{\left(j_{2}\right)}$. The vector $m^{\left(j_{1}\right)}-m^{\left(j_{2}\right)}$, which can be written as

$$
m^{\left(j_{1}\right)}-m^{\left(j_{2}\right)}=\left(\bar{m}^{\left(j_{1}\right)}-\bar{m}^{\left(j_{2}\right)}\right)+\left(m^{\prime\left(j_{1}\right)}-m^{\prime\left(j_{2}\right)}\right),
$$

belongs to $\Gamma_{\theta^{\left(j_{2}\right)}}^{\left(j_{2}+1\right)}$. By (38) , both terms $\bar{m}^{\left(j_{1}\right)}-\bar{m}^{\left(j_{2}\right)}$ and $m^{\prime\left(j_{1}\right)}-m^{\prime\left(j_{2}\right)}$ belong to the face $\Gamma_{\theta^{\left(j_{2}\right)}}^{\left(j_{2}+1\right)} \cap \eta^{\perp}$ of $\Gamma_{\theta^{\left(j_{2}\right)}}^{\left(j_{2}+1\right)}$, and at least one of them is non-zero. 
If $\bar{m}^{\left(j_{1}\right)}-\bar{m}^{\left(j_{2}\right)} \neq 0$ then we get that $\left\langle w, \bar{m}^{\left(j_{1}\right)}-\bar{m}^{\left(j_{2}\right)}\right\rangle=0$. This implies that $\bar{m}^{\left(j_{1}\right)}-\bar{m}^{\left(j_{2}\right)} \in\left(\Gamma_{\theta^{\left(j_{2}\right)}}^{\left(j_{2}+1\right)} \cap \eta^{\perp}\right) \cap w^{\perp}$, but since $w \in \operatorname{int}\left(\theta^{\left(j_{2}\right)}(\eta)\right)$ the face $\Gamma_{\theta^{\left(j_{2}\right)}}^{\left(j_{2}+1\right)} \cap \eta^{\perp} \cap w^{\perp}$ is reduced to zero, a contradiction.

We deduce that $\bar{m}^{(j)}=\bar{m}^{\left(j_{1}\right)}$ for all $j \geq j_{1}$. Since $w \in \operatorname{int}\left(\theta^{(j)}(\eta)\right)$ determines the vertex $\bar{m}^{(j)}$ of the Newton polyhedron of the logarithmic jacobian ideal $\mathcal{J}_{\theta^{(j-1)}}^{(j)}(\eta)$, we can assume, up to relabeling, that

$$
\gamma_{i}^{(j)}=\gamma_{i}^{\left(j_{1}\right)} \text { for }=1, \ldots, d_{0} \text { and } j>j_{1} .
$$

We denote by $\vartheta$ the cone spanned by the vectors $\gamma_{i}^{\left(j_{1}\right)}, \ldots, \gamma_{d_{0}}^{\left(j_{1}\right)}$. We prove below that the cone $\mathbf{R}_{\geq 0}\left(\Gamma_{\theta(j-1)}^{(j)} \cap \eta^{\perp}\right)$ is equal to $\vartheta$ for any $j \geq j_{1}$. This implies that the sequence of cones $\left(\theta^{(j-1)}(\eta)\right)$ stabilizes since $\mathbf{R}_{\geq 0}\left(\Gamma_{\theta(j-1)}^{(j)} \cap \eta^{\perp}\right)$ is the dual cone of $\theta^{(j-1)}(\eta)$.

Otherwise, there exists a vector $\delta^{(j)} \in \Gamma_{\theta^{(j-1)}}^{(j)} \cap \eta^{\perp}$ such that $\delta^{(j)} \notin \vartheta$. Then, we have an expansion of the form:

$$
\delta^{(j)}=a_{1} \gamma_{1}^{\left(j_{1}\right)}+\cdots+a_{d_{0}} \gamma_{d_{0}}^{\left(j_{1}\right)}, \text { with } a_{i} \in \mathbf{Q},
$$

and with some $a_{i}$ less than zero, say $a_{1}$. Since $\gamma_{1}^{\left(j_{1}\right)}=\gamma_{1}^{(j)}$ by (40), we deduce from the definitions that $\delta^{(j+1)}:=\delta_{j}-\gamma_{1}^{(j)}$ belongs to $\Gamma_{\theta^{(j)}}^{(j+1)} \cap \eta^{\perp}$ and $\delta^{(j+1)} \notin \vartheta$. By induction we get that $\delta^{(j+k)}:=\delta_{1}-k \gamma_{1}^{(j)}$ belongs to $\Gamma_{\theta^{(j+k-1)}}^{(j+k)} \cap \eta^{\perp}$ and $\delta^{(j+k)} \notin \vartheta$, for $k>1$. It follows that $\left\langle w, \delta^{(j+k)}\right\rangle=\left\langle w, \delta_{1}\right\rangle-k\left\langle w, \gamma_{1}^{(j)}\right\rangle$ becomes negative for $k \gg 0$. This cannot happen since $w$ belongs to the interior of $\theta^{(j)}(\eta)$, for all $j \gg 0$. This contradiction ends the proof of Proposition 12.31.

The statement of the main result below is given in terms of certain orders on the lattice $M$.

Definition 12.32. A relation $\leq$ on the lattice $M$ is said to be a preorder if it satisfies the following conditions:

- For any $m, n \in M$ one has either $m \leq n$ or $n \leq m$.

- The inequalities $m \leq n$ and $n \leq p$ imply $m \leq p$.

- If $m \leq n$ holds, then $m+p \leq n+p$ for all $p \in M$.

Definition 12.33. If $N$ is the dual lattice of $M$ and if $\underline{\nu}=\left(\nu_{1}, \ldots, \nu_{s}\right) \in\left(N_{\mathbf{R}}\right)^{s}$, for $s \leq d=\mathrm{rkN}$, one can define a preorder $\leq \underline{\underline{\nu}}$ on $M$ by setting for $n, m \in M$ that

$$
n \leq_{\underline{\nu}} m \Leftrightarrow\left(\left\langle n, \nu_{1}\right\rangle, \ldots,\left\langle n, \nu_{s}\right\rangle\right) \leq_{l e x}\left(\left\langle m, \nu_{1}\right\rangle, \ldots,\left\langle m, \nu_{s}\right\rangle\right),
$$

where $\leq_{l e x}$ denotes the lexicographical order on $\mathbf{R}^{s}$. If $\underline{\nu}=\left(\nu_{1}, \ldots, \nu_{d}\right) \in N^{s}$ we say that $\leq_{\underline{\nu}}$ is a rational preorder; if $s=d$ and $\underline{\nu}$ is an ordered basis of $N$ then the rational preorder $\leq_{\underline{\nu}}$ is an order, we say in this case that $\leq_{\underline{\nu}}$ is a rational order.

If $\leq_{\underline{\nu}}$ is a rational order then the relation (41) means that the set of coordinates of $n$ with respect to the dual basis of $\underline{\nu}$ is less than or equal to, for the lexicographic order, the analogous set of coordinates of $m$. If $\nu_{1}=\cdots=\nu_{s}=0$ then the preorder $\leq_{\underline{\nu}}$ is trivial, that is $m \leq_{\underline{\nu}} n \forall m, n \in M$.

Remark 12.34. A basis $\underline{\nu}$ of $N$ defines similarly a valuation of the field $k\left(t^{M}\right)$ of functions of $k\left[t^{M}\right]$, of maximal rank $d$, with values in the totally ordered group $\mathbf{Z}^{d}$ equipped with the lexicographical order. This valuation is defined as the monomial valuation whose value on a monomial $t^{\gamma}$ is $\left(\left\langle\nu_{1}, \gamma\right\rangle, \ldots,\left\langle\nu_{d}, \gamma\right\rangle\right)$.

Let $\underline{\nu}$ be a basis of the lattice $N$ defining the rational order $\leq_{\underline{\nu}}$ on $M$. We denote by $M_{\geq_{\underline{\nu}} 0}$ the set $\left\{m \in M \mid m \geq_{\underline{\nu}} 0\right\}$. If $\Gamma \subset M_{\geq_{\underline{\nu}} 0}$ then we can use the order $\underline{\nu}$ 
to distinguish a $d$-dimensional cone $\tau^{(j)}$ of the the fan $\Sigma^{(j)}$ defining the $j$-th iterated blowing up of the logarithmic jacobian ideal. First, we relabel the minimal system of generators $G=\left\{\gamma_{1}, \ldots, \gamma_{n}\right\}$ of $\Gamma:=\Gamma^{(1)}$ in such a way that $\gamma_{1}=\min _{\leq_{\underline{\nu}}} G$, and then $\gamma_{i}=\min _{\leq \underline{\nu}}\left\{\gamma \in G \mid \gamma_{1} \wedge \ldots \wedge \gamma_{i-1} \wedge \gamma \neq 0\right\}$ for $i=2, \ldots, d$. The semigroup $\Gamma^{(2)}$ is generated by:

$$
\left\{\gamma_{1}, \ldots, \gamma_{d}\right\} \cup\left\{\gamma_{l}-\gamma_{i} \mid 1 \leq i \leq d<l \leq n, \gamma_{1} \wedge \ldots \wedge \gamma_{i-1} \wedge \gamma_{l} \wedge \gamma_{i+1} \wedge \ldots \wedge \gamma_{d} \neq 0\right\} .
$$

The cone $\check{\tau}^{(1)}$ spanned by $\Gamma^{(2)}$ is strictly convex of dimension $d$ and its dual cone $\tau^{(1)}$ belongs to $\Sigma^{(1)}$. With the notations of the begining of Section 12 this means that $\Gamma^{(2)}=\Gamma_{\tau^{(1)}}^{(2)}$ (see Lemma 12.1). By construction the semigroup $\Gamma^{(2)}$ is contained in $M_{\geq \underline{\underline{\nu}} 0}$, hence we can repeat this procedure replacing $G$ by the minimal set of generators $G^{(1)}=\left\{\gamma_{1}^{(1)}, \ldots, \gamma_{n^{(1)}}^{(1)}\right\}$ of the semigroup $\Gamma^{(2)}$. By repeating this procedure inductively, the order $\underline{\nu}$ defines a nested sequence cones $\left(\tau^{(j)}\right)$ of the form (24) and such that $\Gamma^{(j)}=$ $\Gamma_{\tau^{(j-1)}}^{(j)}$ for all $j \geq 1$.

Theorem 12.35. If $\underline{\nu}$ is a basis of $N$ defining a rational order $\leq_{\underline{\nu}}$ such that $\Gamma \subset M_{\geq_{\underline{\nu}} 0}$ then:

(i) For any $j \geq 0$ there exists a unique d-dimensional cone $\tau^{(j)} \in \Sigma^{(j)}$ such that $\Gamma_{\tau^{(j)}}^{(j+1)} \subset M_{\geq \underline{\underline{\nu}} 0}$.

(ii) There exists an integer $l_{0}$, depending on $\underline{\nu}$, such that $\tau^{(j)}=\tau^{\left(l_{0}\right)}$ for $j \geq l_{0}-1$ and the semigroup $\Gamma_{\tau^{\left(l_{0}-1\right)}}^{\left(l_{l}\right)}$ is regular.

Proof. The first assertion is consequence of definitions and the previous discussions.

Suppose that the second assertion of the Theorem does not hold. Then the sequence of cones $\left(\tau^{(j)}\right)$ does not stabilize by Proposition 12.20, By construction we have that the vector $\nu_{1}$ belongs to $\tau^{(j)} \cap N$ for all $j \geq 0$. By Proposition 12.22 there exists an integer $j_{0} \geq 0$ and a cone $\eta$ such that $\nu_{1} \in \operatorname{int}(\eta)$ and $\eta \leq \tau^{(j)}$ for $j \geq j_{0}$. The cone $\eta$ is stable. If the sequence $\left(\tau^{(j)}\right)$ does not stabilize then there exists an integer $j_{1} \geq j_{0}$ and a stable face $\theta$ such that $\eta \leq \theta \leq \tau^{(j)}$ for $j \geq j_{1}$, $\operatorname{codim} \eta=d_{0}>0$ and any face $\vartheta^{(j)} \leq \tau^{(j)}$ such that $\theta \leq \vartheta^{(j)}$ is not stable.

If $\nu \in N$ we denote by $\bar{\nu}$ the canonical image of $\nu$ in $N(\theta)$. Then there exists an integer $1<s \leq d_{0}+1$ such that $\bar{\nu}_{i}=0$ for $i=1, \ldots, s-1$ and $\bar{\nu}_{s} \neq 0$. By definition of the order $\leq_{\underline{\nu}}$ the linear form $\bar{\nu}_{s}$ is non negative on the semigroup $\Gamma_{\tau^{(j-1)}}^{(j)} \cap \theta^{\perp} \subset \Gamma_{\tau^{(j-1)}}^{(j)}$, that is, the vector $\bar{\nu}_{s}$ is a non-zero element of $\tau^{(j-1)}(\theta) \cap N(\theta)$ for $j>j_{1}$.

The vector $\bar{\nu}_{s}$ belongs to the relative interior of a unique face $\zeta^{(j)}(\theta)$ of $\tau^{(j)}(\theta)$ for $j \geq j_{1}$. By Proposition 12.31 there exists an integer $j_{2} \geq j_{1}$ such that $\zeta^{(j)}(\theta)=\zeta^{\left(j_{2}\right)}(\theta)$ for $j \geq j_{2}$. We denote by $\zeta^{(\infty)}(\theta)$ the cone $\zeta^{\left(j_{2}\right)}(\theta)$ and by $d_{1}>0$ its dimension.

For any $j \geq j_{2}$ we fix a maximal chain of faces

$$
0=\zeta_{0}(\theta) \leq \zeta_{1}(\theta) \leq \cdots \leq \zeta_{d_{1}}(\theta)=\zeta^{(\infty)}(\theta),
$$

with $\operatorname{dim} \zeta_{l}(\theta)=l$ for $l=0, \ldots, d_{1}$. Then we get a chain of faces of $\tau^{(j)}$ containing $\theta$ of the form:

$$
\theta=\zeta_{0}^{(j)} \leq \zeta_{1}^{(j)} \leq \cdots \leq \zeta_{d_{1}}^{(j)}
$$

where $\zeta_{l}^{(j)}$ is a face of codimension one of $\zeta_{l+1}^{(j)}$ for $l=0, \ldots, d_{1}-1$. This implies that the sequence $\left(\zeta_{d_{1}}^{(j)}\right)$ is distinguished. By Proposition 12.29 it follows that this sequence stabilizes in a cone, which contains $\theta$ as a proper face. This contradiction ends the proof of Theorem 12.35. 


\section{The Zariski-Riemann Space of A FAn, ACCORding to EWALD-Ishida ([10])}

In this section we recall some properties of a space which plays for proper birational toric maps of toric varieties the role played for proper birational maps of algebraic varieties by the Zariski-Riemann manifold.

As in loc.cit., we denote by $Z R(M)$ the set of all preorders on $M$ (see Definition 12.32). Given $w \in Z R(M)$ we note by $m \leq_{w} n$ the corresponding preorder and by $L(w)$ the subsemigroup $\left\{m \in M \mid m \geq_{w} 0\right\}$. The set $L^{0}(w)=L(w) \cap(-L(w))$ of the elements that are equivalent to zero for the equivalence relation $m={ }_{w} n$ deduced from $\leq_{w}$ is a saturated sublattice of $M$.

By construction, the preorder $\leq_{w}$ induces a total order on the free abelian group $M / L^{0}(w)$ and the canonical quotient map $\lambda(w): M \rightarrow M / L^{0}(w)$ determines the preorder $w$ from this order, so we see that every preorder on $M$ is given by a total order on a free quotient of $M$. We denote by $o(M)$ the trivial preorder corresponding to the case where the free quotient is the zero group.

The topology on $Z R(M)$ is defined by a basis of open sets

$$
(\mathcal{U}(\theta)=\{w \in Z R(M) \mid \check{\theta} \cap M \subset L(w)\})_{\theta}
$$

where $\theta$ runs through the set of rational polyhedral cones in $N_{\mathbf{R}}$. If $\Sigma$ is a rational fan in $N_{\mathbf{R}}$ the Zariski-Riemann manifold of $\Sigma$ is defined to be

$$
Z R(\Sigma)=\bigcup_{\sigma \in \Sigma} \mathcal{U}(\sigma)
$$

By [10], Proposition 2.10, it depends only on the support $|\Sigma|$ of $\Sigma$, and by Theorem 2.4 of loc.cit., it is quasi-compact.

For each $w \in Z R(M)$, since $M / L^{0}(w)$ is a totally ordered group of finite rational rank, it is of finite (real) rank (or height); there is a maximal sequence of distinct convex subgroups

$$
(0)=\Psi_{s}(w) \subset \Psi_{s-1}(w) \subset \cdots \subset \Psi_{1}(w) \subset M / L^{0}(w)
$$

such that the order on $M / L^{0}(w)$ induces on each of its quotients by the $\Psi_{i}(w)$ an order such that the quotient map is monotonous. The totally ordered quotients $M_{i}=$ $M / \lambda(w)^{-1}\left(\Psi_{i}\right)$ corresponding to these subgroups define via the surjections $\lambda_{i}: M \rightarrow M_{i}$ a sequence

$$
w=w_{0}, w_{1}, \ldots, w_{s-1}, w_{s}=o(M)
$$

of preorders on $M$, with which $w$ is said to be composed, as valuations are composed. The integer $s$ can be called the (real) rank or the height of the preorder. To them corresponds a sequence of distinct subsemigroups of $M$ :

$$
L(w) \subset L\left(w_{1}\right) \subset \cdots \subset L\left(w_{s-1}\right) \subset L\left(w_{s}\right)=M .
$$

and a sequence of distinct subgroups of $M$ :

$$
L^{0}(w) \subset L^{0}\left(w_{1}\right) \subset \cdots \subset L^{0}\left(w_{s-1}\right) \subset L^{0}\left(w_{s}\right)=M .
$$

Compare with $\S 3.1$ of [34]. The semigroups $L\left(w_{i}\right)$ are the analogues in this situation of the valuation rings of a field containing a given valuation ring. It is also shown in [10], Lemma 5.1, that for each nontrivial preorder there is an element $y_{0} \in N_{\mathbf{R}}$ such that $L(w) \subset\left\{m \in M \mid\left\langle m, y_{0}\right\rangle \geq 0\right\}$, which is unique up to multiplication by a positive real number. In fact $\left\{x \in M_{\mathbf{R}} \mid\left\langle x, y_{0}\right\rangle \geq 0\right\}$ is the closure in $M_{\mathbf{R}}$ of the convex closure of $L(w)$. To all preorders with which $w$ is composed correspond the same $y_{0}$. It follows from what we have seen that, with the notations introduced above, $L^{0}\left(w_{s-1}\right)$ is the intersection with the lattice $M$ of $\left\{x \in M_{\mathbf{R}} \mid\left\langle x, y_{0}\right\rangle=0\right\}$.

Each quotient $\tilde{S}_{i}=L^{0}\left(w_{i}\right) / L^{0}\left(w_{i-1}\right)(1 \leq i \leq s)$ is a totally ordered lattice of real rank one whose order is given by an embedding into $\mathbf{R}$. The images in $\mathbf{R}$ of the basis vectors 
of this lattice determine in $T_{i \mathbf{R}}$, where $T_{i}$ is the dual lattice of $\tilde{S}_{i}$, a weight vector which can be represented by an element $y_{i} \in N_{\mathbf{R}}$ as in loc.cit. The preorder $w$ on $M$ appears as the composition with $\lambda(w)$ of the lexicographic product of the rank one orders on the quotients $\left(L^{0}\left(w_{i}\right) / L^{0}\left(w_{i-1}\right)\right)_{1 \leq i \leq s}$. Thus, for each preorder $w$ there exist elements $y_{0}, \ldots, y_{s-1}$ in $N_{\mathbf{R}}$ such that $m \geq_{w} 0$ if and only if the sequence $\left(\left\langle y_{0}, m\right\rangle, \ldots,\left\langle y_{s-1}, m\right\rangle\right)$ is $\geq 0$ for the lexicographic order. Each element $y_{i}$ is defined up to positive homothety and modulo the $\mathbf{R}$-vector subspace of $N_{\mathbf{R}}$ generated by the previous ones. In particular, if $w$ is a rank $d$ preorder it is a rational order with respect to some ordered basis of $M$.

Remark 13.1. As a consequence of the discussion above, any preorder $w$ of $M$ is of the form $\leq_{y}$ for $\underline{y}=\left(y_{0}, \ldots, y_{s-1}\right) \in N_{\mathbf{R}}^{d}$ and any rational preorder $w$ of $M$ is of the form $\leq_{\underline{y}}$ for $\bar{y}=\left(\bar{y}_{0}, \ldots, y_{s-1}\right) \in N^{s}$ with $y_{0}, \ldots, y_{s-1}$ linearly independent over $\mathbf{R}$ (compare with Definition 12.33). By induction on the height one can show that any non-trivial preorder is of the form $\leq_{\underline{y}}$ for $\underline{y^{\prime}}=\left(y_{0}^{\prime}, \ldots, y_{s-1}^{\prime}\right)$ and $y_{0}^{\prime}, \ldots, y_{s-1}^{\prime}$ vectors which are part of a basis of $N$.

Note that we can also, just as for valuations, define the rational rank of a preorder $w$ on $M$ : it is the (rational) rank of the free abelian group $M / L^{0}(w)$. The analogue of the residual transcendence degree is then the rank of $L^{0}(w)$, so that for preorders on $M$, the analogue of Abhyankar's equality rat.rankw $+\operatorname{rank} L^{0}(w)=\operatorname{rank} M$ always holds.

A preorder which is an order, which means $L^{0}(w)=0$ is the analogue of a valuation with algebraic residue field extension, also called zero dimensional valuations (their centers are zero dimensional). If we are speaking of valuations dominating a local ring with algebraically closed residue field, they are the valuations with trivial residue field extension, which the second author calls rational valuations in 34. Note also that since the preorders corresponding to vectors of $N$ are dense in $Z R(M)$ for the topology defined by Ewald-Ishida, while the set of orders is closed: if a preorder $w$ is not an order, one choose a rational cone $\theta$ such that $\check{\theta} \cap M \subset L(w)$ and $\check{\theta} \cap L^{0}(w)$ is a non-zero lattice. Then no preorder in the open set $\mathcal{U}(\theta)$, which contains $w$, can be an order.

It is shown in [28] that the closed subset of $Z R(M)$ consisting of orders on $M$, with the induced topology, is homeomorphic to a Cantor set when the rank of the lattice $M$ is $\geq 2$.

To summarize:

- All preorders are "Abhyankar".

- A preorder given by a weight vector $w \in N$ is the analogue of a divisorial valuation on a noetherian local ring. Such preorders are dense.

- A preorder which is an order is the analogue of a zero dimensional valuation. The set of orders is closed in $Z R(M)$ and homeomorphic to a Cantor set.

- A preorder of maximal (real) rank is a rational order with respect to some basis of $N$ and is the analogue of a valuation of real rank equal to the dimension of the ring.

Ewald and Ishida define the fact that a preorder dominates a cone $\theta \subset N_{\mathbf{R}}$ by the following two properties: $\check{\theta} \cap M \subset L(w)$ and $\check{\theta} \cap L^{0}(w)=\theta^{\perp} \cap M$. They show that given a fan $\Sigma$, a given preorder dominates at most one cone of $\Sigma$ ([10], Proposition 2.6) and that one has the inclusion $\check{\theta} \cap M \subset L(w)$ if and only if $w$ dominates a face of $\theta$ ([10], Lemma 2.7), which is then unique.

Denoting by $\operatorname{dom}(\sigma)$ the set of elements $w \in Z R(M)$ dominating a given strictly convex rational polyhedral cone $\sigma \subset N_{\mathbf{R}}$, it is non empty and one has moreover, $F(\sigma)$ 
being the fan consisting of the faces of $\sigma$ :

$$
Z R(F(\sigma))=\bigcup_{\zeta \in F(\sigma)} \operatorname{dom}(\zeta)
$$

If $\sigma$ is a strictly convex cone and $w \in Z R(F(\sigma))$, in each refinement $\Sigma^{(i)}$ of $F(\sigma)$ there is exactly one cone which is dominated by $w$.

Definition 13.2. Given an affine toric variety $T_{F(\sigma)}^{\Gamma}$ as above and a preorder $w$ on $M$ such that $\check{\sigma} \cap M \subset L(w)$, the semigroup of $w$ on $k\left[t^{\Gamma}\right]$ is the image of $\Gamma$ in the quotient $M / L^{0}(w)$; it is a semigroup contained in the positive part of $M / L^{0}(w)$.

If $w$ dominates $\sigma$ this semigroup is the same as the image of $\Gamma$ in the quotient $M / M(\sigma)$ which was used in the proof of Lemma 12.18.

\section{InTERPRETATION OF THE MAIN RESUlt With THE ZARISKI-RIEMANN MANIFOLD OF A FAN}

Now let $\sigma$ be a strictly convex rational cone in $N_{\mathbf{R}}$ and consider the sequence $\Sigma^{(i)}$ of fans obtained by iterating the blowing-up of logarithmic jacobian ideals, with $\Sigma^{(0)}=$ $F(\sigma)$. For each $w \in Z R\left(\Sigma^{(i)}\right)=Z R\left(\Sigma^{(0)}\right)$, and each $i, 0 \leq i<\operatorname{rank}(w)$, the cones dominated by the partial orders $w_{j}$ with which $w$ is composed form a sequence of distinct cones of $\Sigma^{(i)}$

$$
\zeta_{s-1}^{(i)} \subset \zeta_{s-2}^{(i)} \subset \cdots \subset \zeta_{0}^{(i)}
$$

and since $\Sigma^{(i+1)}$ is a refinement of $\Sigma^{(i)}$, we have for each $j, 0 \leq j \leq s-1$ the inclusion $\zeta_{j}^{(i+1)} \subset \zeta_{j}^{(i)}$.

A cone $\zeta \in \Sigma^{(i)}$ is dominated by $w_{s-1}$ if and only if $y_{0}$ is in the relative interior of $\zeta$ (see also [10, the four lines before 2.7, which also show that $\operatorname{dom}(\sigma)$ is not empty).

On the other hand, if $w$ is in fact an order, which means that $L^{0}(w)=0$, then a rational cone $\tau$ dominated by $w$ must satisfy $\check{\tau} \subset L(w)$ and $\operatorname{dim} \tau=\operatorname{rank} M=d$ since we must have $\tau^{\perp}=(0)$; the rank of $w$ must be $\leq d$.

Definition 14.1. Let us keep the notations just introduced. Given a preorder $w \in$ $Z R(F(\sigma))$, we say that the sequence of fans $\Sigma^{(j)}$, or the sequence of toric varieties $T_{\Sigma^{(i)}}^{\Gamma^{(i)}}$, stabilizes at the preorder $w$ if there exists an integer $i \geq 1$ such that the cone $\tau^{(j)}$ of $\Sigma^{(j)}$ dominated by $w$ is regular and the corresponding semigroup is also regular, in particular, it coincides with $\check{\tau}^{(j)} \cap M$.

Proposition 14.2. Given the sequence of fans $\Sigma^{(j)}$ as above, the following statements are equivalent:

(1) There exists an integer $i$ such that the toric variety $T_{\Sigma^{(j)}}^{\Gamma^{(j)}}$ is regular.

(2) For every preorder $w$ of $Z R(F(\sigma))$ the sequence $\Sigma^{(j)}$ stabilizes at $w$.

Proof. We only have to prove that (2) implies (1). By construction if the sequence stabilizes at $w$ and $w$ dominates the regular simplex $\tau^{(j)}$ it also stabilizes for the same index for all preorders $w^{\prime} \in \mathcal{U}\left(\tau^{(j)}\right)$ since the faces of $\tau^{(j)}$ as well as the corresponding semigroups are regular. This gives us an open covering of $Z R\left(\Sigma^{(0)}\right)=Z R(F(\sigma))$. Since it is quasi-compact (see [10], Theorem 2.4) one can extract a finite covering. Taking the maximum $i$ of the indices of stabilization corresponding to those open sets gives us a fan $\Sigma^{(i)}$ covered by regular cones, and so (1).

We can now reformulate the main result as follows, with the usual notations: 
Theorem 14.3. Given an affine toric variety $T^{\Gamma}$ and a rational order $w \in Z R(F(\sigma))$ with respect to some ordered basis of $N$, the sequence $\Sigma^{(i)}$ of the Semple-Nash refinements of the cone $\sigma$ stabilizes for $w$.

Proof. Use the construction at the beginning of this section and Theorem 12.35.

Corollary 14.4. For every rational preorder $w$ of $Z R(F(\sigma))$ the sequence $\Sigma^{(j)}$ stabilizes at $w$.

Proof. By Remark 13.1, the rational preorder $w$ is of the form $\leq_{\underline{\nu}}$, where $\underline{\nu}=\left(\nu_{1}, \ldots, \nu_{s}\right)$ for $s \leq d$ and $\nu_{1}, \ldots, \nu_{s} \in N$ are vectors which belong to a basis of $N$. Then we can complete this sequence to a basis of $N$ and consider the associated rational order $\bar{w}$. It follows that the sequence $\Sigma^{(j)}$ associated to $\bar{w}$ stabilizes at every preorder with which such an order is composed, in particular with $w$.

Remark 14.5. Any preorder given by an integral vector $0 \neq \nu \in N$ is also represented by a primitive vector which generates a direct factor of $M$ so that there exist rational orders which are composed with it. In this case this corollary is the analogue of Hironaka's result in 19 since preorders corresponding to integral vectors are the analogues of divisorial valuations.

Remark 14.6. As a conclusion, we prove the desired result for the restricted class of rational preorders of $Z R(F(\sigma))$, while in order to prove resolution of toric varieties by blowing up logarithmic jacobian ideals one should prove a similar result for all preorders in $Z R(F(\sigma))$.

We end the paper with the verification of the analogue in our situation of Zariski's construction of the Zariski-Riemann space in [40].

Lemma 14.7. Let $w, w^{\prime} \in Z R(F(\sigma))$ be different preorders. There exists a refinement $\Sigma^{\prime}$ of $F(\sigma)$ such that the two cones of $\Sigma^{\prime}$ dominated respectively by $w$ and by $w^{\prime}$ are distinct.

Proof. Since the trivial preorder can dominate only the cone $\{0\}$ in a fan, we may assume that neither preorder is trivial. To $w$ and $w^{\prime}$ let us attach sequences $y_{0}, \ldots, y_{s-1}$ and $y_{0}^{\prime}, \ldots, y_{s^{\prime}-1}^{\prime}$ of elements of $N_{\mathbf{R}}$ as in the previous section. Let us denote by $M^{i}(w)$ the intersection of $M$ with the subspace $\left(\left\langle y_{0}, m\right\rangle=\cdots=\left\langle y_{i-1}, m\right\rangle\right)=0$, with the convention $M^{0}(w)=M$. It is proved in [10] that $w$ dominates a rational convex cone $\tau \subset N_{\mathbf{R}}$ if and only if for $i=0, \ldots, s-1$ we have $y_{i} \in \tau+\left(M^{i}(w)\right)^{\perp}$, and $\check{\tau} \cap M^{s}(w)=M \cap \tau^{\perp}$. The quotients $\tau+\left(M^{i}(w)\right)^{\perp} /\left(M^{i}(w)\right)^{\perp}$ are the dual cones of $\check{\tau} \cap M^{i}(w)_{\mathbf{R}}$. If $y_{0}$ and $y_{0}^{\prime}$ are not homothetic there is certainly a refinement of the fan $F(\sigma)$ in which they are contained in different cones. This also settles the case where $M$ is of rank one. Otherwise we have $M^{1}(w)=M^{1}\left(w^{\prime}\right)$ and the preorders induced by $w$ and $w^{\prime}$ on $M^{1}(w)$ are different. We can now argue by induction on the rank of $M$ since a refinement of the fan $\Sigma+\left(M^{1}(w)\right)^{\perp} /\left(M^{1}(w)\right)^{\perp}$ will induce a refinement of $\Sigma$.

Let us denote by $\left\{T_{\Sigma}^{\Gamma}\right\}$ the set of irreducible $T^{M}(k)$-invariant subvarieties of $T_{\Sigma}^{\Gamma}$ endowed with the topology induced by the Zariski topology. Let us consider all possible equivariant blowing-ups $T_{\Sigma^{\prime}}^{\Gamma^{\prime}} \rightarrow T_{\Sigma}^{\Gamma}$. They form a projective system of $T_{\Sigma^{\Gamma}}^{\Gamma}$-schemes and the $\left\{T_{\Sigma^{\prime}}^{\Gamma^{\prime}}\right\}$ also form a projective system of topological spaces.

Given a preorder $w \in Z R(\Sigma)$ it dominates a cone $\tau^{\prime}$ in each refinement $\Sigma^{\prime}$ of $\Sigma$ and defines an irreducible $T^{M}(k)$-invariant subvariety corresponding to the prime ideal generated by the monomials of $k\left[t^{\Gamma_{\tau}^{\prime}}\right]$ whose exponents are $>_{w} 0$. This defines a map

$$
Z: Z R(\Sigma) \rightarrow \varliminf_{\lim }\left\{T_{\Sigma^{\prime}}^{\Gamma^{\prime}}\right\}
$$


Proposition 14.8. The map $Z$ is an homeomorphism.

Proof. The map is injective by Lemma 14.7, By the construction of the blowing-ups of toric varieties an element $e$ of the projective limit determines in each $T_{\Sigma^{\prime}}^{\Gamma^{\prime}}$ a cone $\tau^{\prime}$ and a semigroup $\Gamma^{\prime} \subset \check{\tau}^{\prime} \cap M$. Let us try to define a preorder on $M$ as follows: $m \geq_{e} 0$ if $m \in \Gamma^{\prime}$ for some $\Gamma^{\prime}$ picked by the element $e$. We have to prove that given $m, n \in M$ either $m-n \geq_{e} 0$ or $n-m \geq_{e} 0$. Since the $\Gamma^{\prime}$ generate $M$ we may assume that $m, n \in \Gamma^{\prime}$ for some $\Gamma^{\prime}$. Consider the ideal generated by $t^{m}, t^{n}$ and let $T_{\Sigma^{\prime \prime}}^{\Gamma^{\prime \prime}} \rightarrow T_{\Sigma^{\prime}}^{\Gamma^{\prime}}$ be its blowing-up. The effect on the fan $\Sigma^{\prime}$ divides the cone $\tau^{\prime}$ in two by the hyperplane dual to the vector $m-n$. The element $e$ picks one of the two charts, say $\tau^{\prime \prime}$, and either $m-n$ or $n-m$ is in the corresponding $\Gamma^{\prime \prime}$, which proves the desired result. The fact that the semigroups $\Gamma^{\prime}$ form an inductive system suffices to show the transitivity of the preorder.

The fact that the map just defined is the inverse of the map $Z$ and determines an homeomorphism follows directly from the definitions. The trivial preorder corresponds to $T^{M} \subset T_{\Sigma^{\prime}}^{\Gamma^{\prime}}$

\section{REFERENCES}

1. A. Atanasov, C. Lopez, A. Perry, N. Proudfoot, and M. Thaddeus, Resolving toric varieties with Nash blow-ups, Experimental Math. 20 (2011), no. 3, 288-303.

2. N. Bourbaki, Éléments de mathématique. VII. Première partie: Les structures fondamentales de l'analyse. Livre II: Algèbre. Chapitre III: Algèbre multilinéaire, Actualités Sci. Ind., no. 1044, Hermann et Cie., Paris, 1948.

3. E. Cattani, R. Curran and A. Dickenstein, Complete intersections in toric ideals, Proc. Amer. Math. Soc. 135 (2007), 329-335.

4. G. Cortiñas, C. Haesemeyer, M. E. Walker and C. A. Weibel Toric varieties, monoid schemes and cdh descent, ArXiv math.KT/11061389, (2011)

5. David A. Cox, John B. Little, and Henry K. Schenck, Toric varieties, Graduate Studies in Mathematics, vol. 124, American Mathematical Society, Providence, RI, 2011.

6. V. I. Danilov, The geometry of toric varieties, Uspekhi Mat. Nauk 33 (1978), no. 2(200), 85-134, 247.

7. D. Duarte, Nash modification on toric surfaces, Revista de la Real Academia de Ciencias Exactas, Físicas y Naturales. Serie A. Matemáticas, first published on line DOI: 10.1007/s13398-012-0104-4.

8. D. Eisenbud and B. Sturmfels, Binomial ideals, Duke Math. J. 84 (1996), no. 1, 1-45.

9. G. Ewald, Combinatorial convexity and algebraic geometry, Graduate Texts in Mathematics, vol. 168, Springer-Verlag, New York, 1996.

10. G. Ewald and M-N Ishida, Completion of real fans and Zariski-Riemann spaces, Tohôku Math. J., (2), 58, (2006), 189-218.

11. W. Fulton, Introduction to toric varieties, Annals of Mathematics Studies, vol. 131, Princeton University Press, Princeton, NJ, 1993, The William H. Roever Lectures in Geometry.

12. I. M. Gel'fand, M. M. Kapranov, and A. V. Zelevinsky, Discriminants, resultants, and multidimensional determinants, Mathematics: Theory \& Applications, Birkhäuser Boston Inc., Boston, MA, 1994.

13. P. D. González Pérez and B. Teissier, Embedded resolutions of not necessarily normal affine toric varieties, C.R. Math. Acad. Sci. Paris 334 (2002), no. 5, 379-382.

14. G. Gonzalez-Sprinberg, Éventails en dimension 2 et transformé de Nash, Publications du Centre de Mathématiques de l'E.N.S., 45, rue d'Ulm, Paris 5e (1977), 1-68.

15. _ Transformé de Nash et éventail de dimension 2, C. R. Acad. Sci. Paris Sér. A-B 284 (1977), no. 1, A69-A71.

16. _ Résolution de Nash des points doubles rationnels, Ann. Inst. Fourier (Grenoble) 32 (1982), no. 2, $\mathrm{x}, 111-178$.

17. D. Grigoriev and P. Milman, Nash desingularization for binomial varieties as Euclidean division, a priori termination bound, polynomial complexity in dim 2, Adv. Math. 231 (2012), no. 6, 3389-3428.

18. R. Hartshorne, Algebraic geometry, Springer-Verlag, New York, 1977, Graduate Texts in Mathematics, No. 52 .

19. H. Hironaka, On Nash blowing-up, Arithmetic and geometry, Vol. II, Progr. Math., vol. 36, Birkhäuser Boston, Mass., 1983, 103-111.

20. K. Kaveh and A. G. Khovanskii, Newton-Okounkov bodies, semigroups of integral points, graded algebras and intersection theory, Annals of Math. 176 (2012), no. 2, 925-978.

21. G. Kempf, F. F. Knudsen, D. Mumford, and B. Saint-Donat, Toroidal embeddings. I, Lecture Notes in Mathematics, Vol. 339, Springer-Verlag, Berlin, 1973.

22. O. Kuroda, The infiniteness of the SAGBI bases for certain invariant rings, Osaka J. Math.,39, (2002), 665-680. 
23. M. Lejeune-Jalabert and A. J. Reguera, The Denef-Loeser series for toric surface singularities, Proceedings of the International Conference on Algebraic Geometry and Singularities (Spanish) (Sevilla, 2001), Rev. Mat. Iberoamericana vol. 19, 2003, 581-612.

24. A. Nobile, Some properties of the Nash blowing-up, Pacific J. Math. 60 (1975), no. 1, 297-305.

25. T. Oda, Torus embeddings and applications, Tata Institute of Fundamental Research Lectures on Mathematics and Physics, vol. 57, Tata Institute of Fundamental Research, Bombay, 1978, Based on joint work with Katsuya Miyake.

26. _ Convex bodies and algebraic geometry, Ergebnisse der Mathematik und ihrer Grenzgebiete (3), vol. 15, Springer-Verlag, Berlin, 1988.

27. J. G. Semple, Some investigations in the geometry of curve and surface elements, Proc. London Math. Soc. (3) 4 (1954), 24-49.

28. A. Sikora, Topology on the spaces of orderings of groups, Bull. London Math. Soc. 36 (2004), no. 4, 519526.

29. M. Spivakovsky, Sandwiched singularities and desingularization of surfaces by normalized Nash transformations, Ann. of Math. (2) 131 (1990), no. 3, 411-491.

30. J. Steiner Über eine besondere Curve dritter Classe (und vierten Grades), Burchardt's Journal Band LIII, 231-237 (Gelesen in der Akademie der Wissenschaften zu Berlin am 7. Januar 1856). Republished in: Steiner, J. (1882). Gesammelte Werke, Band II (Herausgegeben von K. Weierstrass) (Berlin, G. Reimer, 1882), 639647.

31. B. Sturmfels, Gröbner bases and convex polytopes, University Lecture Series, vol. 8, American Mathematical Society, Providence, RI, 1996.

32. H. Sumihiro, Equivariant completion, J. Math. Kyoto Univ. 14 (1974), 1-28.

33. B. Teissier, The hunting of invariants in the geometry of discriminants, Real and complex singularities, Oslo 1976, Sijthoff and Noordhoff International publishers, Alphen aan den Rijn, 1977, 565-677.

34. _ Valuations, deformations, and toric geometry, Valuation theory and its applications, Vol. II (Saskatoon, SK, 1999), Fields Inst. Commun., vol. 33, Amer. Math. Soc., Providence, RI, 2003, 361-459.

35. R. Thom, Sur la théorie des enveloppes, J. Math. Pures Appl. (9), 41, 1962, 177-192.

36. H. M Thompson, Fan is to monoid as scheme is to ring: a generalization of the notion of a fan, ArXiv math.AG/0306221

37. _ Comments on toric varieties, ArXiv math.AG/0310336

38. - Toric singularities revisited, J. Algebra 299 (2006), no. 2, 503-534.

39. H. Whitney, Tangents to an analytic variety, Ann. of Math. (2) 81 (1965), 496-549.

40. O. Zariski, The compactness of the Riemann manifold of an abstract field of algebraic functions, Ann. of Math., 41 (1940), 852-892.

Instituto de Ciencias Matemáticas-CSiC-UAm-UC3M-UCM. Dpto. de Algebra. Facultad de Ciencias Matemáticas. Universidad Complutense de Madrid. Plaza de las Ciencias 3. 28040. Madrid. SPAIN.

E-mail address: pgonzalez@mat.ucm.es

Institut Mathématique de Jussieu, UMR 7586 du CNRS, 175 Rue du Chevaleret, 75013 Paris, France E-mail address: teissier@math.jussieu.fr 